\title{
Public-Supply Pumpage in Kings, Queens, and Nassau Counties, New York, 1880-1995
}

By Anthony Chu, Jack Monti Jr. and Anthony J. Bellitto Jr.

\section{U.S. GEOLOGICAL SURVEY}

Open-File Report 97-567

Prepared in cooperation with the New York City Department of Environmental Protection

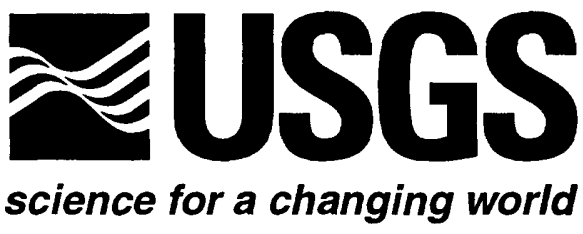

Coram, New York 1997 


\section{U.S. DEPARTMENT OF THE INTERIOR BRUCE BABBITT, Secretary}

U.S. Geological Survey

Mark Shaeffer, Acting Director

For additional information write to:

U.S. Geological Survey 2045 Route 112, Bldg. 4 Coram, NY 11727
Copies of this report may be purchased from:

U.S. Geological Survey Branch of Information Services Box 25286

Denver, CO 80225-0286 


\section{CONTENTS}

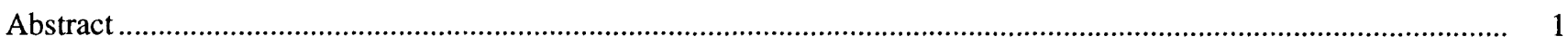

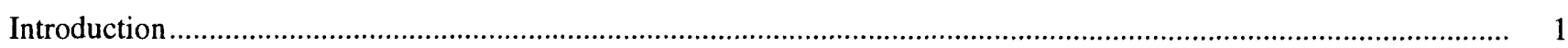

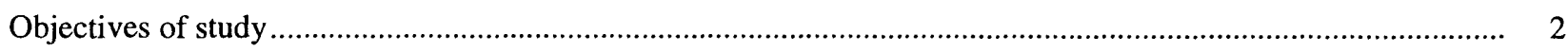

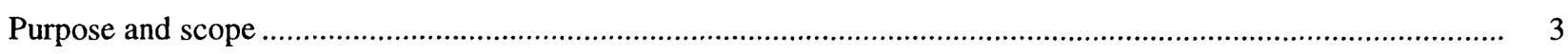

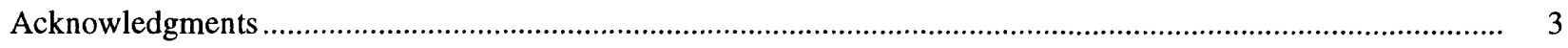

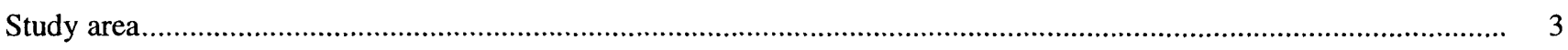

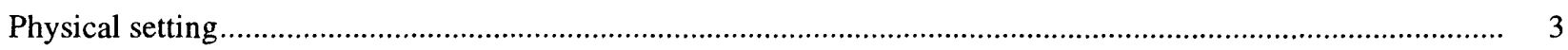

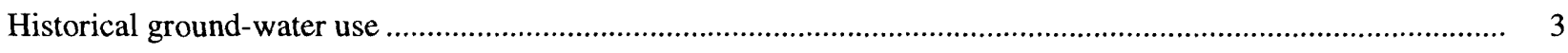

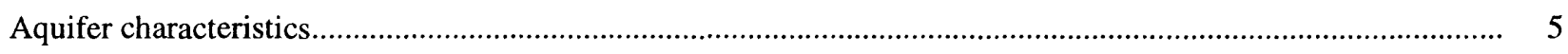

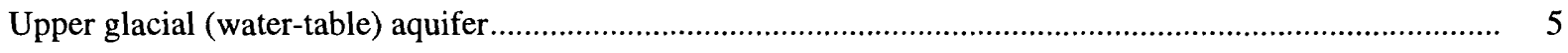

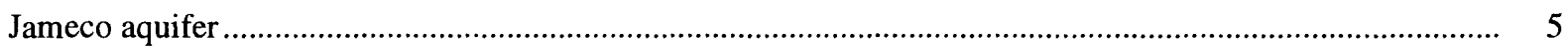

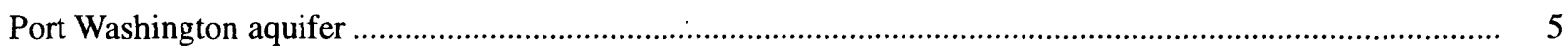

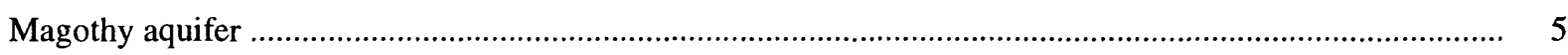

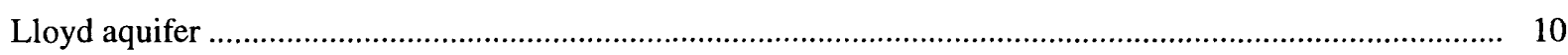

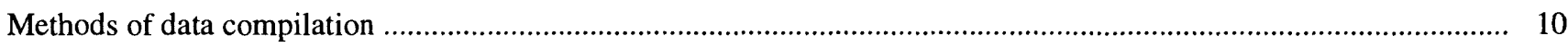

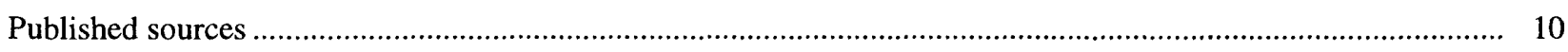

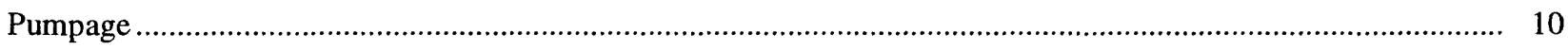

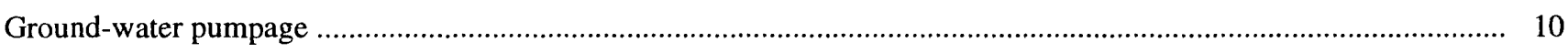

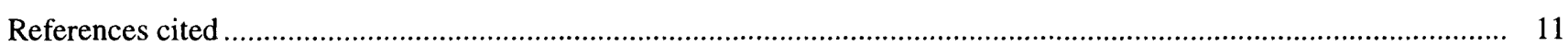

\section{FIGURES}

1. Map showing location and pertinent features of study area, Long Island, N.Y................................................ 2

2. Generalized geologic section showing relative positions of aquifers, Long Island, N.Y....................................

3-6. Graphs of pumpage, 1920-95, by county, Long Island, N.Y.:

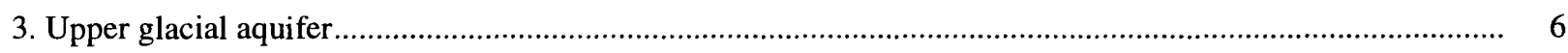

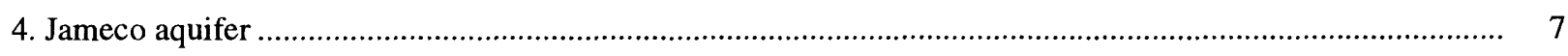

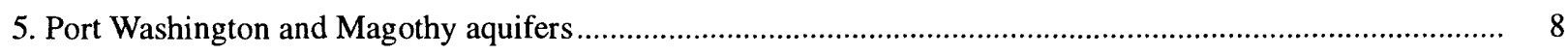

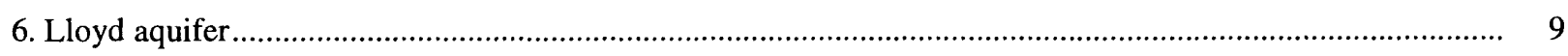

\section{TABLES}

1. Estimated pumpage capacity and reported public-supply pumpage in Kings, Queens, and Nassau Counties,

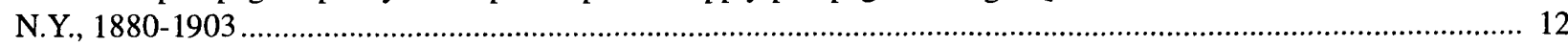

2A. Location and aquifer screened of public-supply wells in Kings, Queens, and Nassau Counties, N.Y...................... 13

2B. Public-supply pumpage in Kings, Queens, and Nassau Counties, N.Y., 1898-1984 ......................................... 21

3. Annual public-supply pumpage totals in Kings, Queens, and Nassau Counties, N.Y., 1920-95, by aquifer and county.....

4. Maximum, minimum, and mean annual pumpage in Kings, Queens, and Nassau Counties, N.Y., 1920-95, by county and aquifer 
CONVERSION FACTORS, ABBREVIATIONS AND VERTICAL DATUM

Multiply

inch (in.)

foot $(\mathrm{ft})$

square mile $\left(\mathrm{mi}^{2}\right)$

gallons per minute (gal/min)

million gallons per day $(\mathrm{Mgal} / \mathrm{d})$

inch per year (in/yr)

feet per day (ft/d)

square foot per day $\left(\mathrm{ft}^{2} / \mathrm{d}\right)$
By

Length

2.54

0.3048

Area

2.59

\section{Flow}

3.7854

0.0438

25.40

Hydraulic conductivity

0.3048

Transmissivity

0.0929
To Obtain

centimeter

meter

square kilometer

liters per minute cubic meters per second millimeter per year

meters per day

square meter per day

Sea level: In this report, "sea level" refers to the National Geodetic Vertical Datum of 1929 (NGVD of 1929) - a geodetic datum derived from a general adjustment of the first-order level nets of the United States and Canada, formerly called Sea Level Datum of 1929. 


\title{
Public-Supply Pumpage in Kings, Queens, and Nassau Counties, New York, 1880-1995
}

\author{
By Anthony Chu, Jack Monti Jr., and Anthony J. Bellitto Jr.
}

\begin{abstract}
Records of public-supply pumpage from wells in Kings, Queens, and Nassau Counties were compiled for use in developing a groundwater flow model of western Long Island and are presented here. Pumpage data from 1880 through 1903 represent 48 well fields, and data from 1898 through 1984 represent 547 wells. These data fill gaps between (1) historical monthly and annual data compiled in a 1981 study by the U.S. Geological Survey, and (2) data provided by the New York State Department of Environmental Conservation, to provide a virtually complete set of public-supply pumpage data from 1880 through 1995. The data include a small number of estimates of annual public-supply pumpage.
\end{abstract}

\section{INTRODUCTION}

Kings and Queens Counties, on western Long Island, N.Y. (fig. 1), obtain nearly all water (about $700 \mathrm{Mgal} / \mathrm{d}$, Buxton and Shernoff, 1995) from an upstate reservoir system operated by the City of New York. The possibility that this source will be inadequate during future droughts or other emergencies has prompted consideration of the underlying aquifer system in Kings and Queens Counties as a supplemental water-supply source. The aquifers beneath the two counties were pumped extensively through the mid1940's until overpumping caused extensive drawdowns and induced saltwater encroachment, which necessitated the shutdown of many wells in several areas. All public-supply pumping was stopped in Kings County (Brooklyn) in 1947 to prevent further saltwater encroachment, and several wells were shut down in western Queens County in 1974 for the same reason.

Pumpage for public and industrial water supply during 1904-47 averaged more than $120 \mathrm{Mgal} / \mathrm{d}$ (Buxton and others, 1981), and drawdowns in the aquifer system in Kings County in 1936 extended to as much as $35 \mathrm{ft}$ below sea level. Within 3 years after the curtailment of pumping in Kings County in 1947, water levels in the water-table aquifer had recovered to within $8 \mathrm{ft}$ of predevelopment levels (Lusczynski, 1952) and have been near predevelopment levels since 1974. Even though a major cone of depression remained in eastern Queens County through the late 1980 's, water levels there did not decline as far as they had in Kings County (Buxton and others, 1981). A major difficulty associated with the water-level recovery is that many of the structures and subways that were constructed when water levels were drawn down, particularly in central and east-central Kings County (fig. 1), now require constant dewatering.

The aquifer system has largely recovered from the effects of the early pumping, and the question has arisen as to whether the system could now safely be used under a monitored pumping regime as a supplement during water-supply shortages. The major concerns are (1) the extent to which intermittent pumping would induce saltwater encroachment, and (2) whether the pumping would induce the migration of contaminants (such as volatile organic compounds [VOC's] and saltwater) from the water-table aquifer to deeper aquifers. The feasibility of using the Kings-Queens aquifer system for water supply will depend on hydraulic characteristics of the aquifers and the chemical quality of the pumped water.

In 1992, the U.S. Geological Survey (USGS), in cooperation with the New York City Department of Environmental Protection, began a 4-year study to develop a ground-water-flow model that can simulate a range of pumping scenarios and indicate what effects they would have on ground-water levels and flow patterns. The results would enable water managers to select optimum rates and locations for pumping to minimize saltwater encroachment and contaminant migration. 


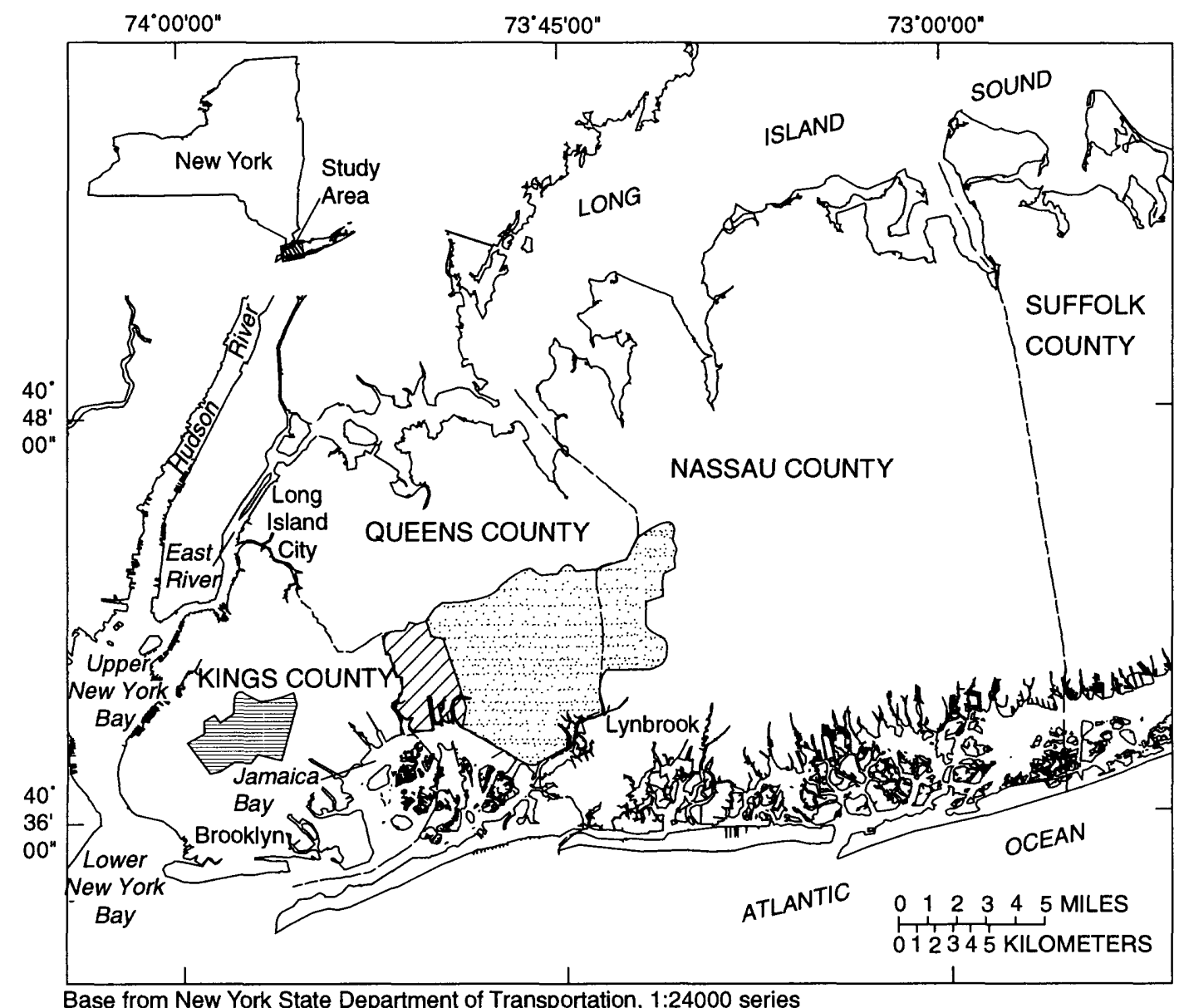

\section{EXPLANATION}

Flatbush pumping center franchise area

Woodhaven pumping center franchise area

Jamaica pumping center franchise area

Figure 1. Location and pertinent features of study area, Long Island, N.Y.

\section{Objectives of Study}

The principal goals of the study were to:

(1) Define the ground-water quality in the KingsQueens aquifer system, with emphasis on contamination from VOC's and saltwater.

(2) Establish a hydrologic-data-collection network in Kings and Queens Counties to obtain data for use in model calibration and future monitoring of water levels and water quality.

(3) Develop a numerical ground-water-flow model to predict the effects of present and proposed water- supply pumping and dewatering operations on ground-water levels and flow patterns.

The work proceeded in five phases:

(1) Reconnaissance of 106 wells that were sampled in Kings, Queens, and Nassau Counties in 1983 (Buxton and Shernoff, 1995). Only 87 of these 106 wells were available for resampling; the others had either been destroyed, had clogged screens, or were inaccessible.

(2) Sampling the remaining wells to locate areas with high concentrations of contaminants. 
(3) Installing 15 wells in three aquifers to provide hydrogeologic information, particularly on the location of the saltwater interface (Chu and Stumm, 1995), and to augment the water-levelmonitoring network.

(4) Compiling pumpage data for use in ground-waterflow model (Nassau County pumpage was included for modeling purposes).

(5) Creating a Geographic Information System (GIS) database for use in the development of the groundwater-flow model. The data elements for each well include screen depth, aquifer, water levels, water chemistry, hydrogeology, pumpage, and specific capacity.

\section{Purpose and Scope}

This report presents supplemental pumpage data that fill gaps between the historical pumpage records (1898-1903) of Veatch and others (1906), and those (1920-79, Nassau County) of Kilburn (1982), and in the recent (1985-95) records of the New York State Department of Environmental Conservation (NYSDEC). The data pertain to wells screened in the upper glacial, Jameco, Port Washington, Magothy, and Lloyd aquifers in Kings, Queens, and Nassau Counties. Total pumpage, by county and aquifer for 192095, as well as maximum, minimum, and average pumpage by county and aquifer, are also presented.

The report also summarizes the history of publicsupply development in the study area and describes the physical and hydrogeological characteristics of the aquifers in the study area; it also explains the methods used to obtain pumpage estimates. Pumpage records from 1880 through 1903 (48 well fields) are derived solely from Veatch and others (1906), are given in table 1, and those from 1898 through 1984 (547 wells) are obtained from USGS and NYSDEC records and are given in table 2B. (Latitude, longitude, and aquifer in which these wells are screened is given in table 2A.) The pumpage data are provided as annual total pumpage by public-supply well or well field, and include a small number of estimates. Tables 1 and $2 B$ are arranged by county, well number, and year. Annual public-supply pumpage totals by county and aquifer, obtained from the NYSDEC for 1925-95, are included in table 3, and maximum, minimum, and mean annual pumpage by county and aquifer are given in table 4 . (All tables at end of report.)

\section{Acknowledgments}

The authors thank Brian Baker of the New York State Department of Environmental Conservation for facilitating the pumpage-data search.

\section{STUDY AREA}

The study area encompasses Kings, Queens, and Nassau Counties in the western part of Long Island and has a total area of about $476 \mathrm{mi}^{2}$ (fig. 1). This area is underlain by four major aquifers-the upper glacial, Jameco, Magothy, and Lloyd (fig. 2).

\section{Physical Setting}

The study area is bounded on the north by Long Island Sound, on the south by the Atlantic Ocean, on the east by Suffolk County, and on the west by the East River, and New York Bay (fig. 1). The southern shore of the study area includes bays, lagoons, islands, and barrier beaches. Precipitation, the sole source of naturally occurring freshwater on Long Island, ranges from 40 to $50 \mathrm{in} / \mathrm{yr}$ (Miller and Frederick, 1969). Altitudes range from $360 \mathrm{ft}$ above sea level on morainal deposits to sea level at the coast. Many streams, and much of the low-lying swamp and marsh areas, were filled in as the area became urbanized.

\section{Historical Ground-Water Use}

The population of the three-county study area increased from less than 2 million in 1900 to more than 5.5 million in 1990 (U.S. Census Bureau, 1990). Before 1880, the water supply in Kings County was derived entirely from streams; Queens and Nassau Counties were still rural and undeveloped. The Kings County water-supply stream network, established in 1862, extended from Brooklyn to Lynbrook (fig. 1) and consisted of six supply ponds that, together, drained $65.6 \mathrm{mi}^{2}$ (Veatch and others, 1906). In 1874, Long Island City (fig. 1) became the first village to use a well for public-water supply; by the 1880 's, Brooklyn was using multiple well stations to supplement the water supply for the rapidly growing population. From then on, ground water was the major source of water supply in the study area. 


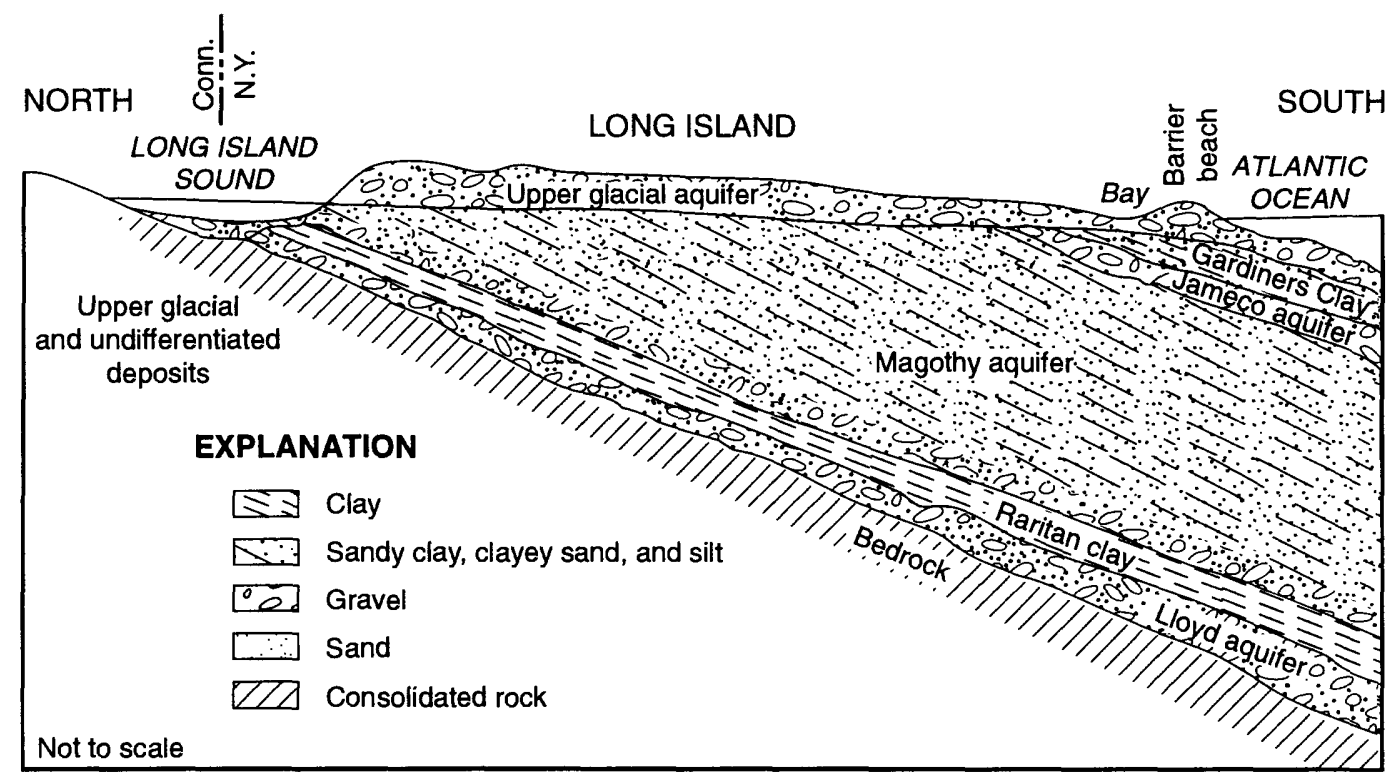

Figure 2. Generalized cross section showing relative positions of aquifers, Long Island, N.Y. (From Franke and McClymonds, 1972, fig. 8.)

In 1904, total ground-water pumpage for public supply was $51 \mathrm{Mgal} / \mathrm{d}-15 \mathrm{Mgal} / \mathrm{d}$ in Kings County, $27 \mathrm{Mgal} / \mathrm{d}$ in Queens, and $9 \mathrm{Mgal} / \mathrm{d}$ in Nassau (Johnson and Waterman, 1952). By 1916, total publicsupply pumpage in the three counties had nearly tripled to $150 \mathrm{Mgal} / \mathrm{d}$, and an estimated additional 75 $\mathrm{Mgal} / \mathrm{d}$ was withdrawn for industrial purposes (Thompson and Leggette, 1936). The first water tunnel from upstate reservoirs was completed in 1917, and ground-water pumpage declined significantly the following year as a result.

From 1918 through 1931, public-supply pumpage in Kings, Queens, and Nassau Counties nearly quadru$\mathrm{pled-from} 46 \mathrm{Mgal} / \mathrm{d}$ to $171 \mathrm{Mgal} / \mathrm{d}$, and industrialsupply pumpage reached historically high levels in Kings and Queens in 1933-50 Mgal/d in Kings County and $25 \mathrm{Mgal} / \mathrm{d}$ in Queens (Thompson and Leggette, 1936). These immense withdrawals prompted the passing of the Water Conservation Law of 1933, which required business owners to return nonconsumptive water pumped at a rate greater than $70 \mathrm{gal} / \mathrm{min}$ back to the source aquifer, rather than discharging it to sewers. Electric refrigeration also contributed to the reduction in ground-water pumpage because water was no longer needed to produce ice for refrigerating food (Buxton and others, 1981). Lusczynski (1952) reported pumpage for ice production to have decreased from 18 $\mathrm{Mgal} / \mathrm{d}$ in 1936 to $4 \mathrm{Mgal} / \mathrm{d}$ in 1947.
By 1947, saltwater intrusion resulting from excessive ground-water withdrawals in Brooklyn had become widespread and led to the shutdown of New York Water Service Corporation's Flatbush wells (fig. 1), where pumpage had averaged over $18 \mathrm{Mgal} / \mathrm{d}$ during 1904-47. The shutdown of the Flatbush wells ended the use of ground water as a public-supply source in Brooklyn.

As Kings County became extensively urban, Queens and Nassau Counties were undergoing rapid population growth and development. Pumpage from the New York Water Service Corporation's Woodhaven wells in Queens County (fig. 1) increased from $45 \mathrm{Mgal} / \mathrm{d}$ in 1950 to over $60 \mathrm{Mgal} / \mathrm{d}$ in the 1970's and, as in Kings County 3 decades earlier, led to saltwater intrusion and the shutdown of the Woodhaven wells in 1974, leaving the Jamaica Water Supply Company (Jamaica pumping center, fig. 1) as the only ground-water public-supply utility remaining in Queens County. Jamaica Water Supply pumpage in Queens exceeded $60 \mathrm{Mgal} / \mathrm{d}$ during 1976-82. A major cone of depression and deteriorating water quality in several wells by the mid-1980's forced the company to reduce pumpage to less than $30 \mathrm{Mgal} / \mathrm{d}$ by the late 1980's (Brian Baker, New York State Department of Environmental Conservation, written commun., 1991).

Since 1948, pumping in Kings County has been only for industrial, commercial, or dewatering pur- 
poses (Buxton and others, 1981). Public-supply pumping continues in eastern Queens at reduced rates, and Nassau County derives all of its public-supply water from wells. Figures 3 through 6 show trends in publicsupply pumpage by aquifer through time. Recent estimates indicate average per-capita water use in Kings and Queens Counties to be about $100 \mathrm{gal} / \mathrm{d}$ per county (Edmund Parrish, New York City Department of Environmental Protection, oral commun., 1996). U.S. Geological Survey estimates show per-capita water use in Kings and Queens Counties to be about $141 \mathrm{gal} / \mathrm{d}$ per county (D.S. Lumia, U.S. Geological Survey, written commun., 1997).

\section{Aquifer Characteristics}

The principal aquifers in the study area are the upper glacial (water table), Magothy, and Lloyd. The Jameco and Port Washington aquifers are local aquifers found along the southern part of the three counties, and the northern part of Nassau County, respectively. The following section briefly describes the geologic and hydraulic characteristics of the aquifers and cites references that provide additional information.

\section{Upper Glacial (Water-Table) Aquifer}

Pleistocene glaciation covered Long Island with a mantle of till, outwash, and glaciolacustrine deposits. Till, a poorly sorted mixture of clay, sand, gravel, and boulders, forms the moraines along northern Long Island and is poorly permeable. Outwash, by contrast, overlies the central and southern part of Long Island and consists of fine to coarse sand and gravel that is moderately to highly permeable and form the upper glacial aquifer (fig. 2). Glaciolacustrine deposits consist of clay, silt, sand, and gravel and are found in central and eastern Long Island, and marine clay deposits are found along the southern shore of Long Island. These deposits are poorly permeable but contain thin, local lenses of sand and gravel that are moderately permeable.

The average horizontal hydraulic conductivity of the Pleistocene deposits ranges from less than $130 \mathrm{ft} / \mathrm{d}$ in till to $270 \mathrm{ft} / \mathrm{d}$ in outwash. The average transmissivity is $24,120 \mathrm{ft}^{2} / \mathrm{d}$ in Kings County, $16,080 \mathrm{ft}^{2} / \mathrm{d}$ in Queens County, 28,140 $\mathrm{ft}^{2} / \mathrm{d}$ in northern Nassau County, and $12,730 \mathrm{ft}^{2} / \mathrm{d}$ in southern Nassau County (McClymonds and Franke, 1972). The upper glacial aquifer is in hydraulic contact with the Magothy except along most of the southern shore of Long Island, where the two aquifers are separated by the Gardiners Clay and Jameco aquifer. The Gardiners Clay restricts vertical flow to underlying aquifers.

\section{Jameco Aquifer}

The Jameco aquifer, a southern-shore glacial stream deposit (fig. 2), lies unconformably beneath the Gardiners Clay throughout most of Kings, Queens, and western Nassau Counties but is in contact with the upper glacial aquifer locally. The Jameco aquifer consists of coarse to fine sand and gravel and is moderately to highly permeable. The average horizontal hydraulic conductivity has been estimated to be $267 \mathrm{ft} / \mathrm{d}$ (Soren, 1971). The average transmissivity is $16,080 \mathrm{ft}^{2} / \mathrm{d}$ in Kings County, $13,400 \mathrm{ft}^{2} / \mathrm{d}$ in Queens County, and $6,700 \mathrm{ft}^{2} / \mathrm{d}$ in southern Nassau County (McClymonds and Franke, 1972).

\section{Port Washington Aquifer}

The Port Washington aquifer, in northern Nassau County, lies unconformably upon bedrock and is overlain by the Port Washington confining unit (Kilburn, 1979). Neither the Port Washington aquifer nor the Port Washington confining unit are shown in figure 2 because their extent is limited. The southern part of the Port Washington aquifer overlaps and abuts the adjacent Cretaceous units. The Port Washington aquifer consists mainly of sand or sand and gravel with interbedded clay, silt, and sandy clay.

\section{Magothy Aquifer}

The Magothy aquifer, of Cretaceous age, lies unconformably beneath the Pleistocene deposits, primarily the upper glacial aquifer, but also Jameco aquifer in southwestern Long Island. The Magothy is underlain unconformably by the Raritan confining unit, hereafter the "Raritan clay," which restricts vertical flow to the underlying Lloyd (fig. 2). The Magothy consists of alternating beds of clay, silt, sand, and gravel and is absent throughout most of Kings County. The average horizontal hydraulic conductivity of the Magothy aquifer has been estimated to be $50 \mathrm{ft} / \mathrm{d}$ (Franke and Cohen, 1972). The average transmissivity is $11,390 \mathrm{ft}^{2} / \mathrm{d}$ where this unit is present in Kings County, and is $10,720 \mathrm{ft}^{2} / \mathrm{d}$ in Queens County, $18,760 \mathrm{ft}^{2} / \mathrm{d}$ in northern Nassau County, and 

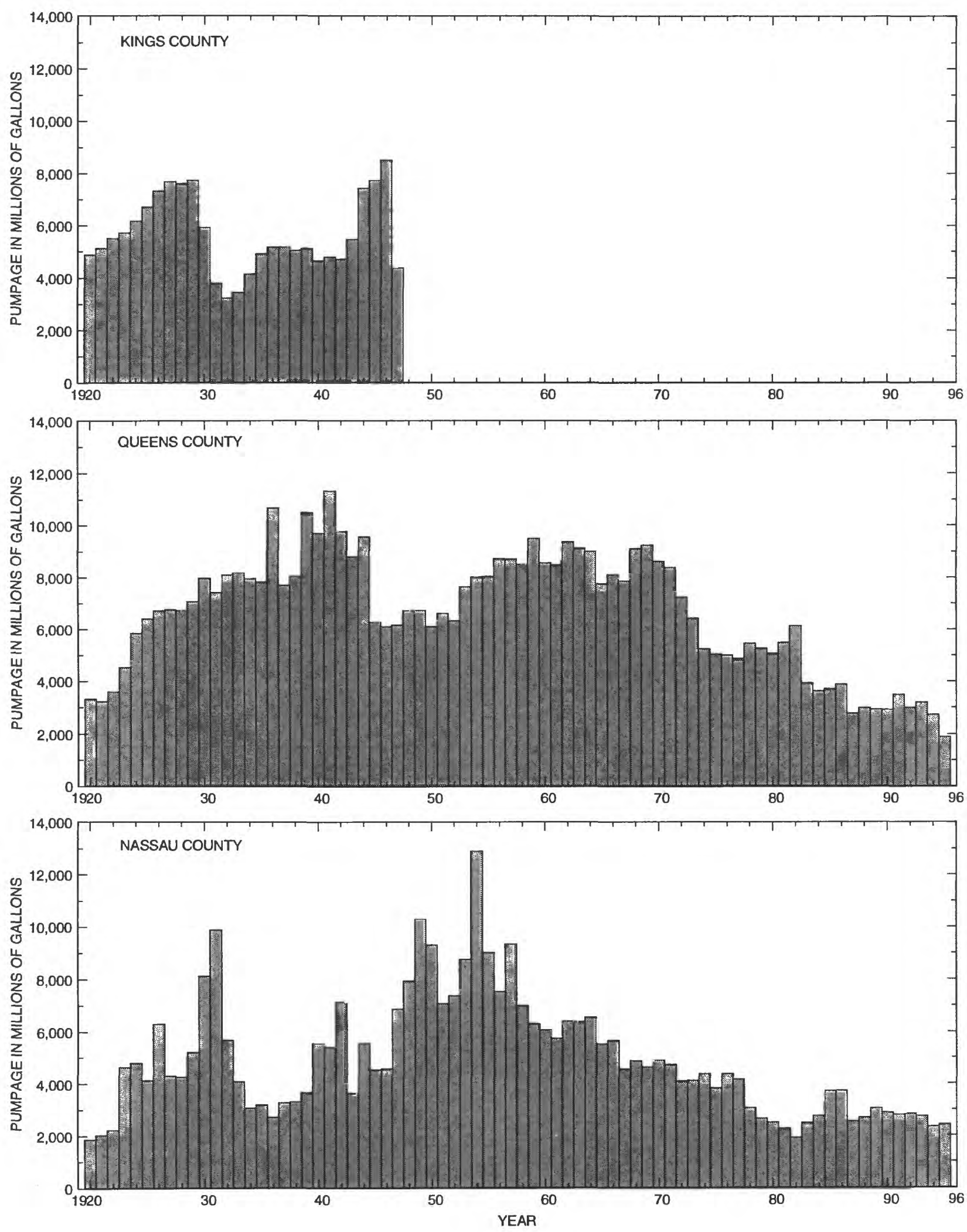

Figure 3. Annual pumpage from upper glacial aquifer in Kings, Queens, and Nassau Counties, N.Y., 1920-95. 

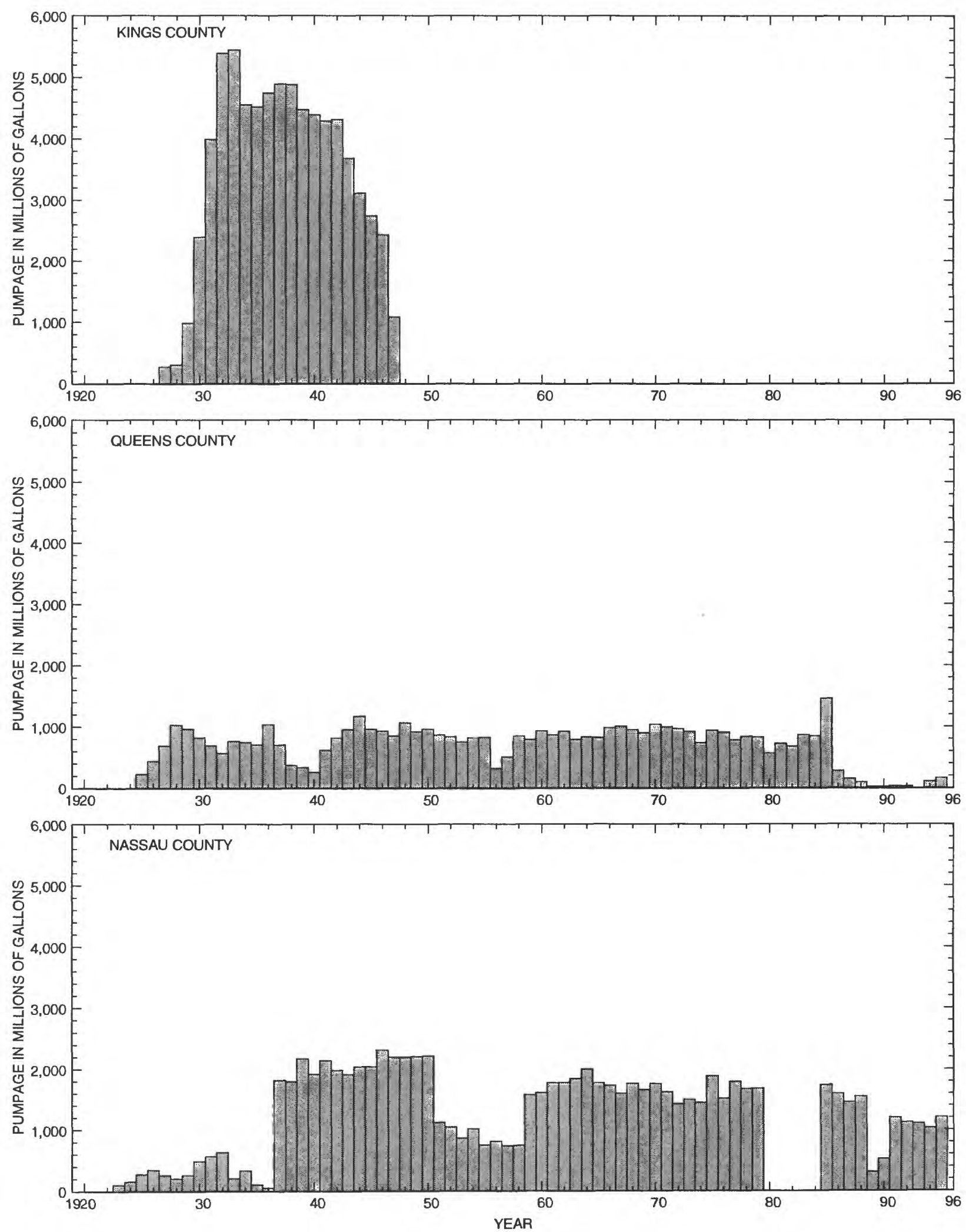

Figure 4. Annual pumpage from Jameco aquifer in Kings, Queens, and Nassau Counties, N.Y., 1920-95. 

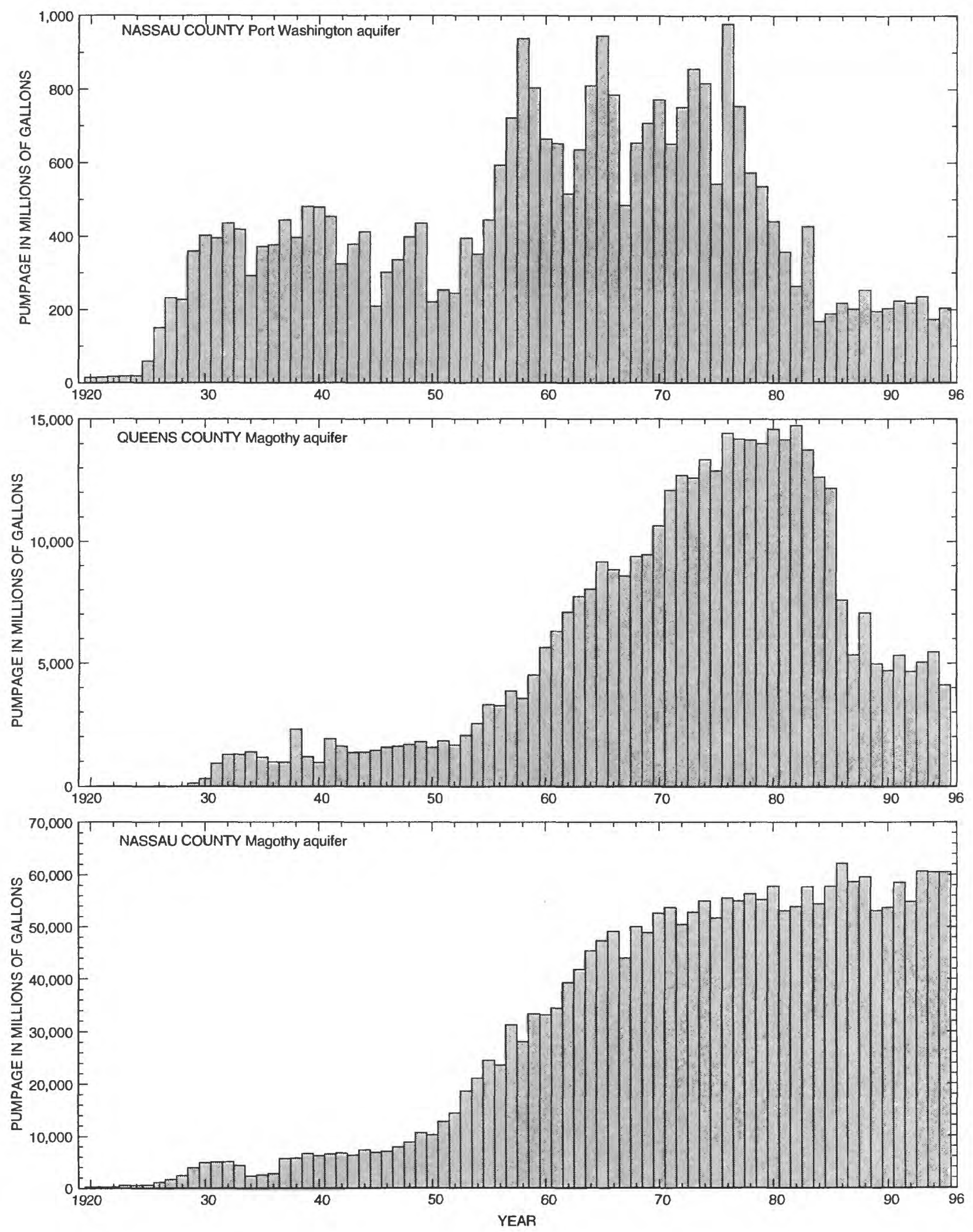

Figure 5. Annual pumpage from Port Washington aquifer in Nassau County, and Magothy aquifer in Queens and Nassau Counties, N.Y., 1920-95. 

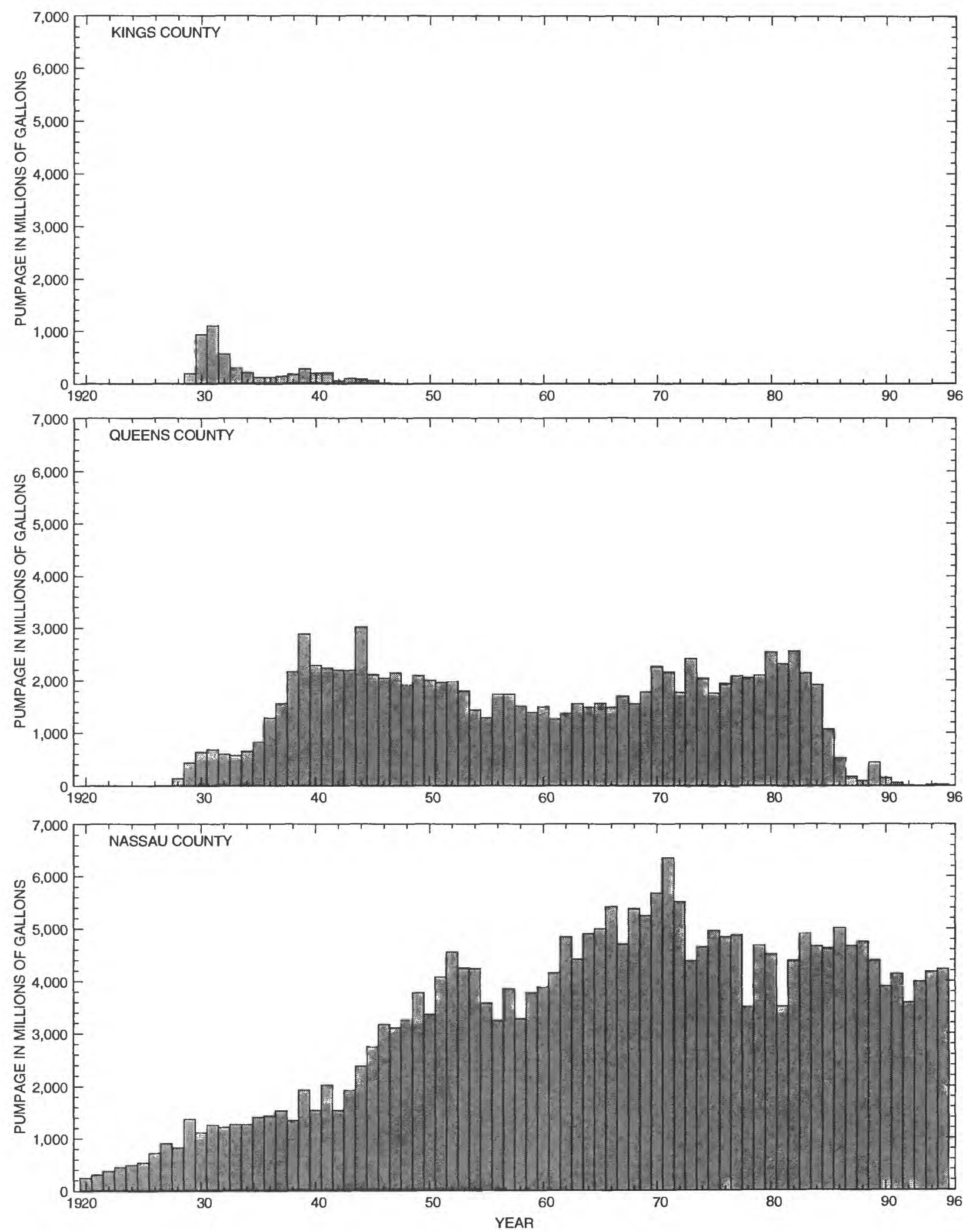

Figure 6. Annual pumpage from Lloyd aquifer in Kings, Queens, and Nassau Counties, N.Y., 1920-95. 
$33,500 \mathrm{ft}^{2} / \mathrm{d}$ in southern Nassau County (McClymonds and Franke, 1972).

\section{Lloyd Aquifer}

The Lloyd aquifer, of the Late Cretaceous-aged Raritan formation, lies conformably beneath the Raritan clay and unconformably upon bedrock (fig. 2). The Lloyd consists of fine to coarse sand and gravel and is poorly to moderately permeable. The average horizontal hydraulic conductivity has been estimated to be 40 $\mathrm{ft} / \mathrm{d}$ (Franke and Cohen, 1972). The average transmissivity is $4,690 \mathrm{ft}^{2} / \mathrm{d}$ in Kings County, $8,040 \mathrm{ft}^{2} / \mathrm{d}$ in Queens County, $12,060 \mathrm{ft}^{2} / \mathrm{d}$ in northern Nassau County, and $16,080 \mathrm{ft}^{2} / \mathrm{d}$ in southern Nassau County.

\section{METHODS OF DATA COMPILATION}

Pumpage values for most years from 1880 through 1903 (table 1) were estimated from data given in Veatch and others (1906) because records were insufficient. Pumpage for 1898 through 1984 (table 2B) was tabulated from USGS and NYSDEC pumpage spreadsheets. Computerized pumpage data for 1985-95 (table 3) were obtained from the NYSDEC at Stony Brook, N.Y., and are tabulated by county and aquifer.

Compilation procedures consisted of (1) review of previous studies, and (2) estimating pumpage for individual wells at multiple-well pumping stations.

\section{Published Sources}

Veatch and others (1906) described the groundwater resources of Long Island and provided a watertable map, as well as a map showing the distribution of pumping centers. Buxton and others (1981) discussed historical pumpage in Kings and Queens Counties.

Kilburn (1982) compiled monthly pumpage records of public-supply wells in Nassau County for 1920-79 and included pumpage from stations in Nassau County that supplied water to New York City. Snavely and Williams (1984) estimated total 1973-79 ground-water withdrawals and returns in Nassau County.

\section{Pumpage}

Pumpage values for 1880 through 1903 were estimated from the multiple-well (well-field) pumping-station data given in Veatch and others (1906) and are listed in table 1. For each well listed, values are given as (1) total well-field-station capacity, and (2) reported (actual) pumpage for a given year, in gallons per day. If no capacity estimate was available for a given station, the highest reported value for that station was used. The well fields contained from 2 wells to more than 100 wells.

Table 2A lists all public-supply wells in the USGS database that have pumpage data and gives their latitude, longitude, and the aquifer in which they are screened. Total annual pumpage, by well, for 1898 through 1984 is given in table 2B. Some water companies record total pumpage of a group of wells instead of by individual well; annual estimates for wells in multiple-well pumping stations were calculated as the total station pumpage divided by the number of wells. The "Remarks" column in table 2B indicates multiple-well pumping stations and whether the pumpage is estimated, and whether monthly data are missing from the annual total. Years without pumpage are not included.

Public-supply pumpage totals by year, county, and aquifer for 1920-95 are given in table 3. Wells with unknown or multiple screened intervals are denoted by the term "unknown" in the "Aquifer" column of table 3. The data in table 3 are depicted as bar graphs in figures 3-6. Maximum, minimum, and mean annual pumpage by county and aquifer for 1920-95 are presented in table 4.

\section{GROUND-WATER PUMPAGE}

Pumpage data are listed by well field and well in tables 1 and 2B. Table 1 lists estimated and actual (reported) average daily pumpage in the three counties by well field for 1880 through 1903 , and table $2 \mathrm{~B}$ lists annual public-supply pumpage, by well, for 1898 through 1984. The 1974-84 data in table 2B were compiled to fill the gap between Kilburn's data (1982) and the NYSDEC's computerized pumpage data for 1985-95; the wells are listed by their New York State well-identification numbers. 


\section{REFERENCES CITED}

Buxton, H.T., and Shernoff, P.K., 1995, Ground-water resources of Kings and Queens Counties, New York: U.S. Geological Survey Open-File Report 92-76, $111 \mathrm{p}$.

Buxton, H.T., Soren, Julian, Posner, Alex, and Shernoff, P.K., 1981, Reconnaissance of the ground-water resources of Kings and Queens Counties, New York: U.S. Geological Survey Open-File Report 81-1186, $64 \mathrm{p}$.

Chu, Anthony, and Stumm, Frederick, 1995, Delineation of the saltwater-freshwater interface at selected locations in Kings and Queens Counties, Long Island, New York, through use of borehole geophysical techniques, in Geology of Long Island and Metropolitan New York, April 22, 1995, Program with Abstracts: Stony Brook, N.Y., Long Island Geologists, p. 21-30.

Franke, O.L., and Cohen, Philip, 1972, Regional rates of ground-water movement on Long Island, New York, in Geological Survey Research 1972: U.S. Geological Survey Professional Paper 800-C, p. C271-277.

Franke, O.L., and McClymonds, N.E., 1972, Summary of the hydrologic situation on Long Island, N.Y., as a guide to water-management alternatives: U.S. Geological Survey Professional Paper 627-F, 59 p.

Johnson, A.H., and Waterman, W.G., 1952, Withdrawal of ground water on Long Island, N.Y.: New York State Water Power and Control Commission Bulletin GW-28, 13 p.

Kilburn, Chabot, 1979, Hydrogeology of the Town of North Hempstead, Nassau County, Long Island, New York: Mineola, N.Y., Nassau County Department of Public Works, Long Island Water Resources Bulletin 12, $87 \mathrm{p}$.
1982, Ground-water pumpage in Nassau County, Long Island, New York 1920-77-introduction and user's guide to the data compilation: U.S. Geological Survey Open-File Report 81-499, 67 p.

Lusczynski, N.J., 1952, The recovery of ground-water levels in Brooklyn, New York, from 1947 to 1950: U.S. Geological Survey Circular 167, 29 p.

McClymonds, N.E., and Franke, O.L., 1972, Watertransmitting properties of aquifers on Long Island, N.Y.: U.S. Geological Survey Professional Paper 627-E, 24 p.

Miller, J.F., and Frederick, R.H., 1969, The precipitation regime of Long Island, N.Y.: U.S. Geological Survey Professional Paper 627-A, 21 p.

Snavely, D.S., and Williams, James, 1984, Estimated public-water supply and industrial-commercial ground-water withdrawals and returns in Nassau County, New York, 1973-79: U.S. Geological Survey Water Resources Investigations Report 84-4246, $16 \mathrm{p}$.

Soren, Julian, 1971, Ground-water and geohydrologic conditions in Queens County, Long Island, N.Y.: U.S. Geological Survey Water-Supply Paper 2001-A, 39 p.

Thompson, D.G., and Leggette, R.M., 1936, Withdrawal of ground water on Long Island, N.Y.: New York State Water Power and Control Commission Bulletin GW1, 28 p.

U.S. Bureau of the Census, 1990, Census geography-concepts and products: Factfinder for the Nation, U.S. Bureau of the Census, CFF (Revised).

Veatch, A.C., Slichter, C.S., Bowman, Isaiah, Crosby, W.O., and Horton, R.E., 1906, Underground water resources of Long Island, New York: U.S. Geological Survey Professional Paper 44, 394 p. 
Table 1. Estimated pumpage capacity and reported public-supply pumpage in Kings, Queens, and Nassau Counties, N.Y., 1880-1903

[Data from Veatch, 1906. Dash indicates data not available; ', degrees; ', minutes; ", seconds]

\begin{tabular}{|c|c|c|c|c|c|c|c|}
\hline \multirow[b]{2}{*}{ Well-field station } & \multirow{2}{*}{ Latitude } & \multirow{2}{*}{ Longitude } & \multirow[b]{2}{*}{ Aquifer } & \multirow{2}{*}{$\begin{array}{l}\text { Year } \\
\text { service } \\
\text { began }\end{array}$} & \multirow{2}{*}{$\begin{array}{l}\text { Estimated station } \\
\text { capacity } \\
\text { (gallons per day) }\end{array}$} & \multicolumn{2}{|c|}{ Recorded pumpage } \\
\hline & & & & & & Gallons per day & Year \\
\hline New Utrecht & 403552 & 735728 & Upper glacial & 1880 & $2,000,000$ & $1,120,596$ & 1899 \\
\hline Gravesend & 403614 & 735721 & Upper glacial & -- & $2,600,000$ & $2,444,032$ & 1899 \\
\hline New Lots & 404012 & 735224 & Upper glacial & 1881 & $4,330,600$ & $4,330,600$ & 1899 \\
\hline Blythebourne & 403757 & 735958 & Upper glacial & 1892 & 200,000 & 200,000 & 1899 \\
\hline Flatbush & 403817 & 735647 & Upper glacial & 1882 & $2,155,400$ & $2,155,400$ & 1899 \\
\hline Spring Creek Old & 404021 & 735120 & Upper glacial & 1882 & $2,500,000$ & $1,986,580$ & 1899 \\
\hline Spring Creek Old & 404021 & 735120 & Jameco & 1882 & $2,500,000$ & $1,986,580$ & 1899 \\
\hline Spring Creek Temp & 404024 & 735131 & Upper glacial & 1894 & $4,500,000$ & $2,997,945$ & 1899 \\
\hline Shetucket & 403951 & 734917 & Jameco & 1897 & $3,500,000$ & $1,678,219$ & 1899 \\
\hline Oconee & 404000 & 734828 & Jameco & 1897 & $2,500,000$ & $1,634,408$ & - \\
\hline Baisley's & 404001 & 734731 & Upper glacial & 1882 & $2,500,000$ & $1,527,051$ & -- \\
\hline Jameco & 404014 & 734715 & Upper glacial & 1888 & $3,000,000$ & $2,467,741$ & 1899 \\
\hline Jameco & 404014 & 734715 & Jameco & 1888 & $3,000,000$ & $2,467,741$ & 1899 \\
\hline Springfield & 403950 & 734535 & Jameco & 1897 & $2,133,890$ & $2,133,890$ & 1899 \\
\hline Forest Stream & 403946 & 734436 & Upper glacial & 1885 & $5,000,000$ & $3,439,039$ & 1899 \\
\hline LI City 1 & 404433 & 735556 & Upper glacial & 1874 & 682,800 & 682,800 & 1899 \\
\hline LI City 2 & 404621 & 735342 & Upper glacial & 1886 & 803,000 & 803,000 & 1899 \\
\hline LI City 3 & 404502 & 735505 & Upper glacial & 1894 & 621,000 & 621,000 & 1899 \\
\hline Bayside-Flushing & 404539 & 734520 & -- & 1874 & $3,000,000$ & $980 ; 000$ & 1899 \\
\hline Whitestone 1 & 404702 & 734919 & Upper glacial & 1892 & $1,000,000$ & 181,000 & 1899 \\
\hline Woodhaven & 404112 & 735104 & Upper glacial & 1894 & 274,000 & 274,000 & 1899 \\
\hline Woodhaven & 404112 & 735104 & Jameco & 1894 & 274,000 & 274,000 & 1899 \\
\hline Montauk & 404151 & 734845 & Upper glacial & 1895 & $1,800,000$ & $1,800,000$ & 1899 \\
\hline Jamaica & 404148 & 734718 & Upper glacial & 1887 & $3,500,000$ & $1,500,000$ & 1899 \\
\hline Jamaica & 404148 & 734718 & Upper glacial & 1887 & $3,500,000$ & $1,137,500$ & 1903 \\
\hline Jamaica & 404148 & 734718 & Jameco & 1887 & $3,500,000$ & $1,137,500$ & 1903 \\
\hline Citizens 1 & 404423 & 735231 & Upper glacial & 1894 & 608,000 & 608,000 & 1899 \\
\hline Citizens 2 & 404525 & 735342 & Upper glacial & 1897 & $1,510,000$ & $1,510,000$ & 1899 \\
\hline Citizens 3 & 404300 & 734942 & Upper glacial & 1899 & $3,500,000$ & $2,067,700$ & 1899 \\
\hline Citizens 4 & 404342 & 735021 & Upper glacial & 1900 & $2,500,000$ & -- & -- \\
\hline Citizens 5 & 404349 & 735029 & Upper glacial & 1901 & $2,000,000$ & -. & -- \\
\hline Bowerybay Northbeach & 404623 & 735326 & Upper glacial & - & 500,000 & 500,000 & 1903 \\
\hline Clear Stream & 403947 & 734305 & Upper glacial & 1885 & $5,000,000$ & $2,568,055$ & 1899 \\
\hline Watts Pond & 403939 & 734229 & Upper glacial & 1894 & $2,500,000$ & $2,213,703$ & 1899 \\
\hline Agawam & 403928 & 733408 & Upper glacial & 1896 & $4,518,951$ & 520,305 & 1899 \\
\hline Merrick & 403938 & 733315 & Upper glacial & 1896 & $4,693,432$ & 325,813 & 1899 \\
\hline Matowa & 403952 & 733220 & Upper glacial & 1896 & $4,495,622$ & 890,939 & 1899 \\
\hline Wantagh & 404010 & 733054 & Upper glacial & 1896 & $3,998,844$ & $1,377,682$ & 1899 \\
\hline Massapequa & 404029 & 732753 & Upper glacial & 1896 & $5,373,196$ & -- & -- \\
\hline Valley Stream & 404002 & 734204 & Upper glacial & 1884 & 561,790 & 561,790 & 1899 \\
\hline Valley Stream & 404002 & 734204 & Jameco & 1884 & 561,790 & 561,790 & 1899 \\
\hline Rockville Centre & 403927 & 733828 & Upper glacial & 1896 & 500,000 & 25,000 & 1897 \\
\hline Rockville Centre & 403927 & 733828 & Upper glacial & 1896 & 500,000 & 150,466 & 1902 \\
\hline Freeport & 403920 & 733536 & Upper glacial & 1894 & 60,000 & 60,000 & 1902 \\
\hline Merrick & 403930 & 733311 & Upper glacial & 1896 & 7,200 & -- & -- \\
\hline Hempstead & 404248 & 733715 & Upper glacial & 1890 & 200,000 & 200,000 & 1897 \\
\hline Hempstead & 404248 & 733715 & Upper glacial & 1890 & 200,000 & 75,000 & 1903 \\
\hline Garden City & 404347 & 733825 & Upper glacial & 1876 & 500,000 & 500,000 & 1897 \\
\hline Sea Cliff & 405027 & 733909 & Upper glacial & 1872 & 500,000 & -- & -- \\
\hline Glen Cove (Pratt) & 405307 & 733759 & Magothy & 1891 & 75,000 & -- & -- \\
\hline Glen Cove (NCWCo.) & 405113 & 733721 & Upper glacial & 1903 & $1,000,000$ & -- & -- \\
\hline
\end{tabular}


Table 2A. Location and aquifer screened of public-supply wells in Kings, Queens, and Nassau Counties, N.Y.

[", degrees; ', minutes; ", seconds; --, no data available]

\begin{tabular}{|c|c|c|c|c|c|c|c|}
\hline $\begin{array}{c}\text { Well } \\
\text { number }\end{array}$ & Latitude & Longitude & $\begin{array}{l}\text { Aquifer in } \\
\text { which well is } \\
\text { screened }\end{array}$ & $\begin{array}{c}\text { Well } \\
\text { number }\end{array}$ & Latitude & Longitude & $\begin{array}{l}\text { Aquifer in } \\
\text { which well is } \\
\text { screened }\end{array}$ \\
\hline${ }^{\top} \mathrm{K} 500$ & 403819 & 735635 & Upper glacial & Q304 & 404025 & 734839 & Upper glacial \\
\hline${ }^{1} \mathrm{~K} 500.2$ & 403819 & 735635 & Upper glacial & Q305 & 404250 & 734538 & Upper glacial \\
\hline K501 & 403819 & 735846 & Upper glacial & Q306 & 404147 & 734718 & Upper glacial \\
\hline K503 & 403836 & 735845 & Upper glacial & Q307 & 404302 & 734513 & Upper glacial \\
\hline K504 & 403819 & 735618 & Upper glacial & Q308 & 404202 & 734916 & Upper glacial \\
\hline K505 & 403815 & 735653 & Upper glacial & Q310 & 404140 & 734412 & Upper glacial \\
\hline K506 & 403920 & 735550 & Upper glacial & Q311 & 404107 & 734805 & Jameco \\
\hline K507 & 403936 & 735614 & Upper glacial & Q312 & 404044 & 734552 & Jameco \\
\hline K508 & 403853 & 735823 & Upper glacial & Q313 & 404330 & 734503 & Upper glacial \\
\hline K510 & 403831 & 735909 & Upper glacial & Q314 & 404049 & 734752 & Jameco \\
\hline K512 & 403842 & 735816 & Upper glacial & Q317 & 404154 & 734937 & Lloyd \\
\hline K513 & 403751 & 735838 & Upper glacial & Q318 & 404254 & 734813 & Magothy \\
\hline K514 & 403830 & 735545 & Upper glacial & Q319 & 404245 & 734734 & Upper glacial \\
\hline K516 & 403951 & 735523 & Upper glacial & Q321 & 404352 & 734448 & Upper glacial \\
\hline K517 & 403950 & 735709 & Jameco & Q322 & 404218 & 734933 & Upper glacial \\
\hline K519 & 403936 & 735613 & Jameco & Q323 & 404200 & 734403 & Upper glacial \\
\hline K520 & 403951 & 735525 & Jameco & Q324 & -- & -. & Upper glacial \\
\hline K521 & 403849 & 735547 & Lloyd & Q324.2 & 404155 & 734638 & Upper glacial \\
\hline K522 & 403857 & 735721 & Jameco & ${ }^{\mathrm{I}} \mathrm{Q} 351$ & 404119 & 735113 & Upper glacial \\
\hline K523 & 403754 & 735813 & Jameco & ${ }^{\mathrm{l}} \mathrm{Q} 352$ & 404005 & 734958 & Upper glacial \\
\hline K524 & 403920 & 735551 & Jameco & Q353 & 404101 & 735102 & Upper glacial \\
\hline K525 & 403818 & 735847 & Jameco & Q354 & 404116 & 735147 & Upper glacial \\
\hline K526 & 403949 & 735737 & Jameco & Q355 & 404122 & 735033 & Upper glacial \\
\hline K527 & 403857 & 735720 & Upper glacial & Q557 & 404223 & 734800 & Magothy \\
\hline K528 & 403921 & 735708 & Jameco & Q558 & 404054 & 734917 & Upper glacial \\
\hline К529 & 403839 & 735847 & -. & Q559 & 404021 & 734839 & Jameco \\
\hline K530 & 403818 & 735810 & Upper glacial & Q560 & 404143 & 734717 & Upper glacial \\
\hline K531 & 403950 & 735740 & -- & Q561 & 404139 & 734715 & Upper glacial \\
\hline K1232 & 403909 & 735756 & Upper glacial & Q562 & 404140 & 734716 & Lloyd \\
\hline K1233 & 403909 & 735757 & Upper glacial & Q563 & 404302 & 734513 & Magothy \\
\hline K1234 & 403909 & 735757 & Upper glacial & Q564 & 404302 & 734513 & Magothy \\
\hline K1329 & 403943 & 735653 & Upper glacial & Q565 & 404201 & 734916 & Lloyd \\
\hline K1331 & 403943 & 735640 & Upper glacial & Q566 & 404154 & 734937 & Magothy \\
\hline K1338 & 403949 & 735737 & Upper glacial & Q567 & 404253 & 734813 & Lloyd \\
\hline K1343 & 403934 & 735539 & Upper glacial & Q568 & 404200 & 734403 & Magothy \\
\hline K1351 & 403921 & 735659 & Upper glacial & Q569 & 404212 & 734642 & Upper glacial \\
\hline K1357 & 403912 & 735830 & Upper glacial & Q570 & 404203 & 734646 & Upper glacial \\
\hline K1359 & 403908 & 735526 & Upper glacial & Q1058 & 404200 & 734638 & Upper glacial \\
\hline K1360 & 403904 & 735628 & Upper glacial & Q1378 & 404120 & 735112 & -- \\
\hline K1363 & 403923 & 735527 & Upper glacial & Q1379 & 404154 & 735107 & -- \\
\hline K1516 & 403943 & 735653 & Upper glacial & Q1450 & 404207 & 734459 & Upper glacial \\
\hline Q68 & 404108 & 735032 & Upper glacial & Q1476 & 404041 & 734956 & Upper glacial \\
\hline Q301 & 404214 & 734934 & Upper glacial & Q1477 & 404050 & 735022 & -- \\
\hline Q302 & 404223 & 734801 & Upper glacial & Q1484 & 404113 & 735011 & Upper glacial \\
\hline Q303 & 404054 & 734917 & Upper glacial & Q1493 & 404140 & 735041 & Upper glacial \\
\hline
\end{tabular}

TLatitude and longitude given for this well are at approximate center of well field.

${ }^{2}$ Well not listed in table $2 \mathrm{~B}$. 
Table 2A. Location and aquifer screened of public-supply wells in Kings, Queens, and Nassau Counties, N.Y.--continued

\begin{tabular}{|c|c|c|c|c|c|c|c|}
\hline $\begin{array}{c}\text { Well } \\
\text { number }\end{array}$ & $\underset{0}{\text { Latitude }}$ & Longitude & $\begin{array}{l}\text { Aquifer in } \\
\text { which well is } \\
\text { screened }\end{array}$ & $\begin{array}{c}\text { Well } \\
\text { number }\end{array}$ & Latitude & Longitude & $\begin{array}{l}\text { Aquifer in } \\
\text { which well is } \\
\text { screened }\end{array}$ \\
\hline Q1511 & 404139 & 735105 & Upper glacial & Q3034 & 404234 & 734553 & Magothy \\
\hline Q1528 & 404137 & 735158 & Upper glacial & Q3062 & 404059 & 734508 & Magothy \\
\hline Q1534 & 404249 & 734435 & Upper glacial & Q3069 & 404202 & 734917 & Lloyd \\
\hline Q1600 & 404330 & 734503 & Magothy & Q3083 & 404056 & 734406 & Magothy \\
\hline Q1604 & 404152 & 735052 & Upper glacial & ${ }^{2} \mathrm{Q} 3156$ & 404050 & 734755 & - \\
\hline Q1629 & 404249 & 734435 & Magothy & ${ }^{2} \mathrm{Q} 3157$ & 404107 & 734805 & -. \\
\hline Q174் & 404323 & 734553 & Upper glacial & ${ }^{2} \mathrm{~N} 10$ & 404229 & 734246 & Magothy \\
\hline Q1811 & 404151 & 734917 & Upper glacial & ${ }^{2} \mathrm{~N} 11$ & 404224 & 734238 & Magothy \\
\hline Q1815 & 404211 & 734500 & Magothy & $\mathrm{N} 12$ & 404219 & 734240 & Magothy \\
\hline Q1839 & 404150 & 734719 & Upper glacial & $\mathrm{N} 13$ & 404214 & 734241 & Magothy \\
\hline Q1840 & 404057 & 734854 & Upper glacial & $\mathrm{N} 14$ & 404411 & 734137 & Upper glacial \\
\hline Q1843 & 404145 & 734734 & Upper glacial & N15 & 404427 & 734149 & Upper glacial \\
\hline Q1957 & 404250 & 734538 & Magothy & $\mathrm{N} 17$ & 404437 & 734023 & Upper glacial \\
\hline Q1958 & 404140 & 734412 & Magothy & $\mathrm{N} 22$ & 404642 & 734405 & Magothy \\
\hline Q1997 & 404248 & 734601 & Upper glacial & ${ }^{2} \mathrm{~N} 23$ & 404642 & 734405 & Lloyd \\
\hline Q2000 & 404332 & 734429 & Magothy & N24. & 404735 & 734242 & Lloyd \\
\hline Q2001 & 404259 & 734634 & Upper glacial & $\mathrm{N} 28$ & 404833 & 734147 & Upper glacial \\
\hline Q2006 & 404216 & 734912 & Upper glacial & N29 & 404830 & 734148 & Upper glacial \\
\hline Q2026 & 404042 & 734336 & Magothy & ${ }^{2} \mathrm{~N} 30$ & 404901 & 734430 & Port Washington \\
\hline Q2027 & 404156 & 734525 & Magothy & N31 & 404857 & 734427 & Port Washington \\
\hline Q2028 & 404156 & 734525 & Magothy & ${ }^{2} \mathrm{~N} 33$ & 405011 & 734150 & Port Washington \\
\hline Q2137 & 404254 & 734813 & Magothy & ${ }^{2} \mathrm{~N} 35$ & 405010 & 734151 & Port Washington \\
\hline Q2138 & 404204 & 735000 & Upper glacial & ${ }^{2} \mathrm{~N} 36$ & 405109 & 734303 & Port Washington \\
\hline Q2188 & 404332 & 734429 & Magothy & N37 & 405111 & 734302 & Upper glacial \\
\hline Q2189 & 404123 & 734930 & Upper glacial & ${ }^{2} \mathrm{~N} 40$ & 403536 & 733941 & Lloyd \\
\hline Q2243 & 404116 & 734521 & Upper glacial & ${ }^{2} \mathrm{~N} 41$ & 403536 & 733936 & Lloyd \\
\hline Q2275 & 404216 & 734423 & Upper glacial & ${ }^{2} \mathrm{~N} 42$ & 403537 & 733939 & Magothy \\
\hline Q2276 & 404216 & 734423 & Magothy & ${ }^{2} \mathrm{~N} 43$ & 403531 & 734012 & Lloyd \\
\hline Q2299 & 404225 & 734503 & Upper glacial & ${ }^{2} \mathrm{~N} 44$ & 403531 & 734023 & Lloyd \\
\hline Q2300 & 404224 & 734503 & Magothy & ${ }^{2} \mathrm{~N} 45$ & 403536 & 733943 & Lloyd \\
\hline Q2321 & 404245 & 734406 & Upper glacial & N46 & 403534 & 733527 & Lloyd \\
\hline Q2332 & 404204 & 735000 & Magothy & ${ }^{2} \mathrm{~N} 48$ & 403923 & 733922 & Magothy \\
\hline Q2343 & 404249 & 734406 & Magothy & ${ }^{2} \mathrm{~N} 49$ & 403923 & 733916 & Magothy \\
\hline Q2362 & 404320 & 734818 & Magothy & ${ }^{2} \mathrm{~N} 50$ & 403923 & 733916 & Magothy \\
\hline Q2363 & 404343 & 734831 & Magothy & ${ }^{2} \mathrm{~N} 52$ & 403929 & 733826 & Magothy \\
\hline Q2373 & 404323 & 734838 & Magothy & N68 & 403922 & 733532 & Magothy \\
\hline Q2374 & 404323 & 734838 & Magothy & N69 & 403921 & 733537 & Magothy \\
\hline Q2408 & 404329 & 734827 & Magothy & $\mathrm{N} 72$ & 404104 & 733741 & Magothy \\
\hline Q2409 & 404329 & 734827 & Magothy & N75 & 404138 & 733836 & Upper glacial \\
\hline Q2432 & 404247 & 734603 & Magothy & N76 & 404136 & 733833 & Upper glacial \\
\hline Q2435 & 404351 & 734448 & Magothy & ${ }^{2} \mathrm{~N} 78$ & 404259 & 733716 & Magothy \\
\hline Q2442 & 404135 & 734402 & Upper glacial & N79 & 404252 & 733714 & Magothy \\
\hline Q2443 & 404135 & 734402 & Magothy & N80 & 404256 & 733712 & Magothy \\
\hline Q2955 & 404040 & 734450 & Magothy & N81 & 404305 & 733709 & Magothy \\
\hline Q3014 & 404309 & 734700 & Magothy & N82 & 404308 & 733707 & Magothy \\
\hline${ }^{2} \mathrm{Q} 3029$ & 404059 & 734508 & -- & N83 & 404306 & 733713 & Lloyd \\
\hline
\end{tabular}


Table 2A. Location and aquifer screened of public-supply wells in Kings, Queens, and Nassau Counties, N.Y.--continued

\begin{tabular}{|c|c|c|c|c|c|c|c|}
\hline $\begin{array}{c}\text { Well } \\
\text { number }\end{array}$ & Latitude & Longitude & $\begin{array}{l}\text { Aquifer in } \\
\text { which well is } \\
\text { screened }\end{array}$ & $\begin{array}{c}\text { Well } \\
\text { number }\end{array}$ & Latitude & Longitude & $\begin{array}{l}\text { Aquifer in } \\
\text { which well is } \\
\text { screened }\end{array}$ \\
\hline${ }^{2} \mathrm{~N} 91$ & 404350 & 733816 & Upper glacial & ${ }^{2} \mathrm{~N} 715$ & 404047 & 733547 & Upper glacial \\
\hline${ }^{2} \mathrm{~N} 93$ & 404352 & 733831 & Upper glacial & ${ }^{2}$ N728 & 404049 & 732559 & Upper glacial \\
\hline N94 & 404350 & 733825 & Magothy & ${ }^{2} \mathrm{~N} 729$ & 404057 & 732603 & Upper glacial \\
\hline N95 & 404352 & 733830 & Magothy & ${ }^{2}$ N733 & 405230 & 733230 & Port Washington \\
\hline N97 & 404448 & 733812 & Magothy & ${ }^{2} \mathrm{~N} 735$ & 405231 & 733232 & Upper glacial \\
\hline${ }^{2}$ N98 & 404446 & 733813 & Magothy & ${ }^{2} \mathrm{~N} 736$ & 405230 & 733231 & Upper glacial \\
\hline N101 & 404521 & 733534 & Magothy & ${ }^{2} \mathrm{~N} 737$ & 404223 & 734238 & Upper glacial \\
\hline N103 & 404553 & 733830 & Magothy & ${ }^{2} \mathrm{~N} 746$ & 404432 & 732900 & Magothy \\
\hline N104 & 404557 & 733828 & Magothy & ${ }^{2} \mathrm{~N} 747$ & 404432 & 732902 & Magothy \\
\hline${ }^{2} \mathrm{~N} 105$ & 404652 & 733727 & Magothy & ${ }^{2} \mathrm{~N} 750$ & 404525 & 733532 & Magothy \\
\hline${ }^{2}$ N107 & 404733 & 733524 & Magothy & ${ }^{2} \mathrm{~N} 751$ & 403934 & 733409 & Upper glacial \\
\hline${ }^{2} \mathrm{~N} 109$ & 404925 & 733817 & Lloyd & ${ }^{2} \mathrm{~N} 757$ & 403934 & 733411 & Upper glacial \\
\hline${ }^{2} \mathrm{~N} 110$ & 404931 & 733821 & Lloyd & ${ }^{2} \mathrm{~N} 801$ & 405113 & 733727 & Upper glacial \\
\hline${ }^{2} \mathrm{~N} 112$ & 405114 & 733727 & Upper glacial & ${ }^{2} \mathrm{~N} 802$ & 405113 & 733726 & Upper glacial \\
\hline${ }^{2} \mathrm{~N} 115$ & 405242 & 733516 & Port Washington & ${ }^{2} \mathrm{~N} 808$ & 405114 & 733727 & Upper glacial \\
\hline${ }^{2} \mathrm{~N} 116$ & 405242 & 733523 & Upper glacial & ${ }^{2} \mathrm{~N} 819$ & 405010 & 734143 & Upper glacial \\
\hline${ }^{2} \mathrm{~N} 117$ & 405240 & 733522 & Upper glacial & ${ }^{2} \mathrm{~N} 824$ & 405010 & 734143 & Port Washington \\
\hline N118 & 405243 & 733510 & Lloyd & N835 & 405346 & 733753 & Lloyd \\
\hline N119 & 405243 & 733524 & Lloyd & ${ }^{2} \mathrm{~N} 871$ & 403937 & 733321 & Upper glacial \\
\hline${ }^{2} \mathrm{~N} 131$ & 403949 & 733417 & Magothy & ${ }^{2} \mathrm{~N} 872$ & 403939 & 733320 & Upper glacial \\
\hline N132 & 403951 & 733418 & Magothy & ${ }^{2} \mathrm{~N} 890$ & 403940 & 733315 & Magothy \\
\hline N133 & 403953 & 733420 & Magothy & ${ }^{2} \mathrm{~N} 901$ & 405034 & 734012 & Upper glacial \\
\hline N134 & 403950 & 733415 & Magothy & ${ }^{2} \mathrm{~N} 906$ & 405035 & 733912 & Lloyd \\
\hline${ }^{2} \mathrm{~N} 148$ & 404441 & 733207 & Magothy & ${ }^{2} \mathrm{~N} 952$ & 403940 & 733316 & Upper glacial \\
\hline${ }^{2} \mathrm{~N} 149$ & 404626 & 733112 & Upper glacial & ${ }^{2} \mathrm{~N} 953$ & 403939 & 733320 & Upper glacial \\
\hline${ }^{2} \mathrm{~N} 150$ & 404629 & 733111 & Upper glacial & ${ }^{2} \mathrm{~N} 1039$ & 403951 & 734306 & Upper glacial \\
\hline N152 & 404628 & 733418 & Magothy & ${ }^{2} \mathrm{~N} 1041$ & 403952 & 734314 & Magothy \\
\hline${ }^{2} \mathrm{~N} 166$ & 405148 & 733417 & Magothy & N1298 & 404655 & 734445 & Lloyd \\
\hline${ }^{2} \mathrm{~N} 192$ & 404432 & 732901 & Magothy & N1328 & 404713 & 734105 & Lloyd \\
\hline N198 & 404917 & 732929 & Magothy & ${ }^{2} \mathrm{~N} 1329$ & 404525 & 733533 & Magothy \\
\hline N199 & 404922 & 732924 & Magothy & ${ }^{2} \mathrm{~N} 1344$ & 404046 & 733547 & Upper glacial \\
\hline${ }^{2}$ N525 & 404410 & 732616 & Upper glacial & N1402 & 403951 & 733616 & Upper glacial \\
\hline N570 & 404919 & 732931 & Magothy & ${ }^{2} \mathrm{~N} 1489$ & 403850 & 734241 & Upper glacial \\
\hline${ }^{2}$ N578 & 404456 & 733913 & Magothy & ${ }^{2} \mathrm{~N} 1491$ & 403847 & 734240 & Upper glacial \\
\hline${ }^{2} \mathrm{~N} 580$ & 404002 & 733332 & Upper glacial & ${ }^{2} \mathrm{~N} 1502$ & 403900 & 734245 & Upper glacial \\
\hline${ }^{2} \mathrm{~N} 585$ & 405230 & 733231 & Upper glacial & N1595 & 405033 & 733909 & Upper glacial \\
\hline${ }^{2} \mathrm{~N} 613$ & 405148 & 733417 & Magothy & N1601 & 404046 & 733546 & Magothy \\
\hline N650 & 404533 & 733936 & Magothy & N1602 & 404029 & 733935 & Magothy \\
\hline N651 & 404534 & 733933 & Magothy & N1603 & 404112 & 733935 & Magothy \\
\hline N687 & 404743 & 734444 & Lloyd & N1618 & 404631 & 734215 & Lloyd \\
\hline N693 & 404229 & 734244 & Upper glacial & N1651 & 405231 & 733633 & Lloyd \\
\hline${ }^{2} \mathrm{~N} 698$ & 404137 & 733428 & Upper glacial & ${ }^{2} \mathrm{~N} 1667$ & 404524 & 733532 & Magothy \\
\hline N700 & 404642 & 734406 & Upper glacial & ${ }^{2} \mathrm{~N} 1696$ & 404407 & 733538 & Magothy \\
\hline${ }^{2} \mathrm{~N} 703$ & 404736 & 734242 & Upper glacial & N1697 & 404357 & 733837 & Magothy \\
\hline${ }^{2} \mathrm{~N} 705$ & 404410 & 732616 & Upper glacial & N1715 & 404907 & 734111 & Lloyd \\
\hline${ }^{2} \mathrm{~N} 706$ & 404411 & 732616 & Upper glacial & N1716 & 404911 & 734111 & Lloyd \\
\hline${ }^{2} \mathrm{~N} 710$ & 403852 & 734238 & Jameco & $\mathrm{N} 1802$ & 404512 & 734210 & Lloyd \\
\hline${ }^{2} \mathrm{~N} 711$ & 403852 & 734237 & Jameco & N1870 & 404811 & 733912 & Magothy \\
\hline
\end{tabular}


Table 2A. Location and aquifer screened of public-supply wells in Kings, Queens, and Nassau Counties, N.Y.--continued

\begin{tabular}{|c|c|c|c|c|c|c|c|}
\hline $\begin{array}{c}\text { Well } \\
\text { number }\end{array}$ & $\underset{0}{\text { Latitude }}$ & Longitude & $\begin{array}{l}\text { Aquifer in } \\
\text { which well is } \\
\text { screened }\end{array}$ & $\begin{array}{c}\text { Well } \\
\text { number }\end{array}$ & $\underset{0}{\text { Latitude }}$ & Longitude & $\begin{array}{l}\text { Aquifer in } \\
\text { which well is } \\
\text { screened }\end{array}$ \\
\hline N1871 & 404811 & 733912 & Magothy & ${ }^{2} \mathrm{~N} 3193$ & 404338 & 733040 & Magothy \\
\hline $\mathrm{N} 1872$ & 404811 & 733912 & Magothy & ${ }^{2} \mathrm{~N} 3194$ & 404338 & 733046 & Magothy \\
\hline N1873 & 404811 & 733911 & Magothy & ${ }^{2} \mathrm{~N} 3312$ & 404310 & 733029 & Magothy \\
\hline N1874 & 404810 & 733911 & Magothy & ${ }^{2} \mathrm{~N} 3313$ & 404309 & 733026 & Upper glacial \\
\hline N1875 & 404810 & 733912 & Magothy & ${ }^{2} \mathrm{~N} 3327$ & 404033 & 734312 & Magothy \\
\hline N1876 & 404810 & 733912 & Magothy & $\mathrm{N} 3427$ & 404050 & 732948 & Magothy \\
\hline N1877 & 404810 & 733912 & Lloyd & N3443 & 404815 & 734345 & Lloyd \\
\hline${ }^{2} \mathrm{~N} 1879$ & 404642 & 734405 & Lloyd & ${ }^{2} \mathrm{~N} 3448$ & 403511 & 734150 & Lloyd \\
\hline${ }^{2} \mathrm{~N} 1880$ & 404642 & 734405 & Magothy & N3456 & 404302 & 733325 & Magothy \\
\hline${ }^{2} \mathrm{~N} 1881$ & 404642 & 734406 & Lloyd & N3457 & 404308 & 733320 & Magothy \\
\hline${ }^{2} \mathrm{~N} 1882$ & 404642 & 734405 & Magothy & ${ }^{2} \mathrm{~N} 3463$ & 404132 & 733114 & Magothy \\
\hline${ }^{2} \mathrm{~N} 1893$ & 404638 & 734413 & Magothy & N3465 & 404305 & 733331 & Magothy \\
\hline${ }^{2} \mathrm{~N} 1900$ & 404642 & 734405 & Upper glacial & ${ }^{2} \mathrm{~N} 3466$ & 405113 & 733727 & Upper glacial \\
\hline${ }^{2} \mathrm{~N} 1902$ & 404637 & 734418 & Upper glacial & N3474 & 404847 & 733440 & Magothy \\
\hline${ }^{2} \mathrm{~N} 1927$ & 403520 & 733819 & Lloyd & N3475 & 404849 & 733445 & Magothy \\
\hline N1937 & 404409 & 732711 & Magothy & ${ }^{2} \mathrm{~N} 3488$ & 404445 & 733104 & Magothy \\
\hline N1958 & 404426 & 734148 & Lloyd & N3520 & 404112 & 733935 & Magothy \\
\hline${ }^{2} \mathrm{~N} 1971$ & 403922 & 733537 & Upper glacial & N3523 & 404814 & 734112 & Magothy \\
\hline N2028 & 404731 & 734007 & Magothy & $\mathrm{N} 3540$ & 404823 & 734148 & Upper glacial \\
\hline $\mathrm{N} 2030$ & 404907 & 734109 & Magothy & ${ }^{2} \mathrm{~N} 3552$ & 404454 & 733203 & Magothy \\
\hline $\mathrm{N} 2052$ & 404829 & 733953 & Magothy & ${ }^{2} \mathrm{~N} 3553$ & 404453 & 733246 & Magothy \\
\hline${ }^{2} \mathrm{~N} 2066$ & 404545 & 732841 & Magothy & ${ }^{2} \mathrm{~N} 3561$ & 405230 & 733230 & Upper glacial \\
\hline${ }^{2} \mathrm{~N} 2072$ & 404634 & 733111 & Upper glacial & ${ }^{2} \mathrm{~N} 3564$ & 404051 & 732948 & Upper glacial \\
\hline $\mathrm{N} 2115$ & 404106 & 734329 & Upper glacial & N3603 & 404248 & 734023 & Magothy \\
\hline N2214 & 404826 & 734504 & Lloyd & N3604 & 404247 & 734023 & Magothy \\
\hline${ }^{2} \mathrm{~N} 2236$ & 404519 & 733427 & Magothy & N3605 & 404153 & 734102 & Magothy \\
\hline N2239 & 404133 & 733839 & Upper glacial & N3618 & 404338 & 733046 & Magothy \\
\hline $\mathrm{N} 2400$ & 404727 & 733804 & Magothy & N3668 & 404153 & 733733 & Magothy \\
\hline${ }^{2} \mathrm{~N} 2402$ & 404400 & 733147 & Upper glacial & N3672 & 404459 & 734021 & Magothy \\
\hline${ }^{2} \mathrm{~N} 2403$ & 404359 & 733141 & Upper glacial & ${ }^{2} \mathrm{~N} 3673$ & 404459 & 734023 & Magothy \\
\hline $\mathrm{N} 2413$ & 404125 & 734210 & Magothy & N3680 & 404134 & 733114 & Magothy \\
\hline N2414 & 404124 & 734210 & Upper glacial & N3687 & 403536 & 733943 & Lloyd \\
\hline${ }^{2} \mathrm{~N} 2487$ & 404546 & 733902 & Magothy & N3695 & 404347 & 733612 & Magothy \\
\hline N2565 & 404434 & 733940 & Magothy & N3696 & 404351 & 733613 & Magothy \\
\hline $\mathrm{N} 2578$ & 404033 & 734312 & Upper glacial & N3697 & 404352 & 733611 & Magothy \\
\hline N2580 & 404323 & 733145 & Upper glacial & N3698 & 404352 & 733607 & Magothy \\
\hline${ }^{2} \mathrm{~N} 2581$ & 404323 & 733137 & Upper glacial & N3704 & 404132 & 733834 & Upper glacial \\
\hline N2597 & 403532 & 734034 & Lloyd & N3720 & 404112 & 734041 & Magothy \\
\hline $\mathrm{N} 2602$ & 404518 & 733433 & Lloyd & N3722 & 404048 & 733547 & Upper glacial \\
\hline $\mathrm{N} 2613$ & 403951 & 733615 & Magothy & N3732 & 404644 & 733839 & Magothy \\
\hline${ }^{2} \mathrm{~N} 2747$ & 404446 & 733650 & Magothy & N3733 & 404628 & 733831 & Magothy \\
\hline N2748 & 404445 & 733651 & Magothy & N3745 & 404105 & 733747 & Magothy \\
\hline N2920 & 405257 & 732930 & Lloyd & N3780 & 404228 & 732935 & Upper glacial \\
\hline${ }^{2} \mathrm{~N} 3142$ & 404545 & 732841 & Magothy & N3781 & 404030 & 734145 & Magothy \\
\hline${ }^{2} \mathrm{~N} 3147$ & 404546 & 732841 & Magothy & N3782 & 404030 & 734146 & Magothy \\
\hline N3185 & 404416 & 733847 & Magothy & N3832 & 404049 & 733544 & Magothy \\
\hline
\end{tabular}


Table 2A. Location and aquifer screened of public-supply wells in Kings, Queens, and Nassau Counties, N.Y.--continued

\begin{tabular}{|c|c|c|c|c|c|c|c|}
\hline $\begin{array}{c}\text { Well } \\
\text { number }\end{array}$ & Latitude & Longitude & $\begin{array}{l}\text { Aquifer in } \\
\text { which well is } \\
\text { screened }\end{array}$ & $\begin{array}{c}\text { Well } \\
\text { number }\end{array}$ & Latitude & Longitude & $\begin{array}{l}\text { Aquifer in } \\
\text { which well is } \\
\text { screened }\end{array}$ \\
\hline N3876 & 404352 & 732911 & Magothy & N4512 & 404100 & 734122 & Magothy \\
\hline N3878 & 404624 & 733233 & Magothy & $\mathrm{N} 4602$ & 404154 & 732720 & Magothy \\
\hline N3881 & 404320 & 734025 & Magothy & N4623 & 404722 & 733948 & Magothy \\
\hline N3892 & 405233 & 733724 & Upper glacial & N4756 & 404206 & 733452 & Magothy \\
\hline N3893 & 404228 & 732933 & Magothy & N4757 & 404210 & 733454 & Magothy \\
\hline N3894 & 404059 & 733417 & Magothy & N4758 & 404209 & 733448 & Magothy \\
\hline N3895 & 404119 & 733231 & Magothy & N4759 & 404205 & 733447 & Magothy \\
\hline N3905 & 404544 & 734151 & Magothy & N4859 & 405010 & 734141 & Port Washington \\
\hline N3934 & 404402 & 733708 & Magothy & N4860 & 405010 & 734147 & Upper glacial \\
\hline N3935 & 404400 & 733705 & Magothy & N5007 & 404552 & 733420 & Magothy \\
\hline N3937 & 404001 & 734020 & Magothy & N5099 & 404647 & 734235 & Magothy \\
\hline N3953 & 404628 & 733237 & Magothy & N5121 & 403958 & 734103 & Magothy \\
\hline${ }^{2} \mathrm{~N} 4042$ & 404309 & 732745 & Upper glacial & N5145 & 404034 & 734311 & Magothy \\
\hline N4043 & 404309 & 732749 & Magothy & N5147 & 404214 & 732620 & Magothy \\
\hline${ }^{2} \mathrm{~N} 4063$ & 404532 & 732849 & Magothy & N5148 & 404311 & 732747 & Magothy \\
\hline N4077 & 404323 & 734138 & Upper glacial & N5152 & 405326 & 733514 & Port Washington \\
\hline N4082 & 404525 & 733732 & Magothy & N5153 & 403942 & 733644 & Magothy \\
\hline N4095 & 404639 & 732809 & Magothy & N5155 & 404238 & 734203 & Upper glacial \\
\hline N4096 & 404639 & 732802 & Magothy & N5156 & 404238 & 734203 & Magothy \\
\hline N4097 & 404631 & 732939 & Magothy & N5163 & 404400 & 733859 & Magothy \\
\hline N4118 & 404129 & 733838 & Upper glacial & N5187 & 404041 & 733438 & Magothy \\
\hline N4132 & 403942 & 733644 & Magothy & N5193 & 403929 & 733821 & Magothy \\
\hline${ }^{2} \mathrm{~N} 4133$ & 404809 & 733034 & Magothy & N5194 & 403923 & 733922 & Magothy \\
\hline${ }^{2} \mathrm{~N} 4146$ & 404533 & 732848 & Magothy & N5195 & 403923 & 733916 & Magothy \\
\hline N4206 & 404524 & 733632 & Magothy & N5201 & 404925 & 733817 & Lloyd \\
\hline N4223 & 404855 & 734034 & Upper glacial & N5209 & 404941 & 734030 & Upper glacial \\
\hline N4243 & 404541 & 734152 & Magothy & N5227 & 403532 & 733534 & Lloyd \\
\hline N4245 & 404746 & 733211 & Magothy & N5259 & 404119 & 733227 & Magothy \\
\hline${ }^{2} \mathrm{~N} 4246$ & 404802 & 733128 & Magothy & N5260 & 404136 & 733837 & Magothy \\
\hline N4265 & 404755 & 733724 & Magothy & ${ }^{2} \mathrm{~N} 5301$ & 404427 & 733150 & Magothy \\
\hline N4298 & 404323 & 734138 & Magothy & N5302 & 404246 & 733143 & Magothy \\
\hline N4327 & 404621 & 733923 & Magothy & N5303 & 404252 & 733004 & Magothy \\
\hline${ }^{2} \mathrm{~N} 4342$ & 404011 & 733105 & Upper glacial & ${ }^{2} \mathrm{~N} 5304$ & 404225 & 733043 & Magothy \\
\hline${ }^{2} \mathrm{~N} 4367$ & 404011 & 733105 & Upper glacial & N5308 & 403519 & 733828 & Lloyd \\
\hline N4388 & 404646 & 734403 & Magothy & N5318 & 404154 & 733453 & Magothy \\
\hline N4389 & 405056 & 734109 & Upper glacial & N5319 & 404154 & 733450 & Magothy \\
\hline N4390 & 404514 & 734121 & Magothy & N5320 & 404155 & 733447 & Magothy \\
\hline N4393 & 403929 & 734123 & Magothy & N5321 & 404242 & 733157 & Magothy \\
\hline${ }^{2} \mathrm{~N} 4394$ & 404001 & 734020 & Magothy & N5322 & 404243 & 733202 & Magothy \\
\hline N4400 & 405154 & 732958 & Magothy & ${ }^{2} \mathrm{~N} 5336$ & 404441 & 733207 & Magothy \\
\hline N4405 & 403515 & 734305 & Lloyd & ${ }^{2} \mathrm{~N} 5468$ & 403955 & 734341 & Jameco \\
\hline N4411 & 403920 & 734043 & Magothy & N5484 & 404419 & 733643 & Magothy \\
\hline N4425 & 404259 & 733715 & Magothy & N5485 & 404423 & 733655 & Magothy \\
\hline N4447 & 404311 & 733325 & Magothy & ${ }^{2} \mathrm{~N} 5486$ & 404415 & 733655 & Magothy \\
\hline $\mathrm{N} 4448$ & 404307 & 733328 & Magothy & N5528 & 404728 & 734005 & Magothy \\
\hline N4450 & 404323 & 733136 & Magothy & N5596 & 404454 & 733726 & Magothy \\
\hline N4451 & 404429 & 733055 & Magothy & N5603 & 404517 & 734023 & Magothy \\
\hline N4461 & 404051 & 732948 & Magothy & N5653 & 404108 & 733716 & Magothy \\
\hline
\end{tabular}


Table 2A. Location and aquifer screened of public-supply wells in Kings, Queens, and Nassau Counties, N.Y.--continued

\begin{tabular}{|c|c|c|c|c|c|c|c|}
\hline $\begin{array}{c}\text { Well } \\
\text { number }\end{array}$ & Latitude & Longitude & $\begin{array}{l}\text { Aquifer in } \\
\text { which well is } \\
\text { screened }\end{array}$ & $\begin{array}{c}\text { Well } \\
\text { number }\end{array}$ & $\underset{0}{\text { Latitude }}$ & Longitude & $\begin{array}{l}\text { Aquifer in } \\
\text { which well is } \\
\text { screened }\end{array}$ \\
\hline${ }^{2} \mathrm{~N} 5654$ & 404451 & 733526 & Magothy & N6915 & 404401 & 732831 & Magothy \\
\hline N5655 & 404541 & 733335 & Magothy & N6916 & 404358 & 732831 & Magothy \\
\hline N5656 & 403948 & 733928 & Magothy & N6945 & 404547 & 734011 & Magothy \\
\hline N5695 & 403922 & 733543 & Magothy & N6956 & 404556 & 732705 & Magothy \\
\hline N5696 & 403945 & 733415 & Magothy & N7030 & 404635 & 733311 & Magothy \\
\hline N5703 & 404156 & 732620 & Magothy & N7058 & 404318 & 734011 & Magothy \\
\hline N5710 & 404559 & 734155 & Magothy & N7076 & 404339 & 733040 & Magothy \\
\hline N5762 & 405129 & 733615 & Magothy & N7104 & 404832 & 733722 & Magothy \\
\hline N5767 & 404054 & 732948 & Magothy & N7117 & 404214 & 734059 & Magothy \\
\hline N5792 & 405014 & 733736 & Upper glacial & N7126 & 404652 . & 734007 & Magothy \\
\hline${ }^{2} \mathrm{~N} 5801$ & 404032 & 732800 & Magothy & N7157 & 405058 & 734111 & Upper glacial \\
\hline${ }^{2} \mathrm{~N} 5807$ & 404032 & 732800 & Upper glacial & N7298 & 404303 & 733714 & Magothy \\
\hline${ }^{2} \mathrm{~N} 5848$ & 404057 & 732603 & Magothy & N7353 & 404552 & 733416 & Magothy \\
\hline N5852 & 404808 & 733746 & Magothy & N7377 & 404313 & 732750 & Magothy \\
\hline N5876 & 404858 & 734115 & Upper glacial & N7407 & 404002 & 733332 & Magothy \\
\hline N5884 & 404756 & 734258 & Magothy & N7414 & 404054 & 732613 & Magothy \\
\hline N5947 & 404646 & 733908 & Magothy & N7421 & 404554 & 732706 & Magothy \\
\hline N6045 & 404432 & 733656 & Magothy & N7445 & 404514 & 734121 & Magothy \\
\hline N6076 & 404650 & 732911 & Magothy & N7446 & 404848 & 733443 & Magothy \\
\hline N6077 & 404649 & 732910 & Magothy & N7482 & 404109 & 734329 & Magothy \\
\hline N6078 & 404535 & 732848 & Magothy & N7512 & 404536 & 734103 & Magothy \\
\hline N6087 & 405010 & 734143 & Upper glacial & N7513 & 404652 & 733727 & Magothy \\
\hline N6092 & 404912 & 732751 & Magothy & N7515 & 404337 & 732707 & Magothy \\
\hline N6093 & 404908 & 732751 & Magothy & N7516 & 404337 & 732705 & Magothy \\
\hline N6146 & 404005 & 733916 & Magothy & N7521 & 403948 & 733927 & Magothy \\
\hline N6148 & 404216 & 732733 & Magothy & N7522 & 404005 & 733916 & Magothy \\
\hline N6149 & 404215 & 732622 & Magothy & N7523 & 404309 & 733026 & Magothy \\
\hline N6150 & 404245 & 732903 & Magothy & N7526 & 404703 & 732801 & Magothy \\
\hline N6190 & 404707 & 733053 & Magothy & N7548 & 404010 & 734252 & Magothy \\
\hline N6191 & 404707 & 733049 & Magothy & N7549 & 404738 & 733531 & Magothy \\
\hline N6192 & 404517 & 733102 & Magothy & N7551 & 404656 & 733946 & Magothy \\
\hline${ }^{2} \mathrm{~N} 6193$ & 404517 & 733105 & Magothy & N7552 & 404649 & 733944 & Magothy \\
\hline N6315 & 404526 & 733626 & Magothy & N7561 & 404453 & 733246 & Magothy \\
\hline N6442 & 404123 & 732852 & Magothy & N7562 & 404634 & 733111 & Magothy \\
\hline N6443 & 404123 & 732852 & Magothy & N7593 & 405045 & 732830 & Magothy \\
\hline N6450 & 403532 & 734010 & Lloyd & N7620 & 405424 & 733400 & Lloyd \\
\hline N6580 & 404630 & 732938 & Magothy & N7643 & 405424 & 733400 & Upper glacial \\
\hline N6644 & 404408 & 732713 & Magothy & N7649 & 404344 & 734121 & Magothy \\
\hline N6651 & 404754 & 733157 & Magothy & N7650 & 404344 & 734121 & Magothy \\
\hline N6744 & 404238 & 734205 & Upper glacial & N7651 & 404611 & 734010 & Magothy \\
\hline N6745 & 404239 & 734202 & Magothy & N7665 & 405203 & 733500 & Upper glacial \\
\hline N6817 & 403929 & 733817 & Magothy & N7720 & 404237 & 733952 & Magothy \\
\hline N6819 & 404537 & 733335 & Magothy & N7747 & 404736 & 734242 & Upper glacial \\
\hline N6866 & 404039 & 732835 & Magothy & N7748 & 404735 & 734242 & Upper glacial \\
\hline N6867 & 404039 & 732835 & Magothy & N7749 & 404735 & 734242 & Upper glacial \\
\hline N6893 & 404049 & 733544 & Magothy & N7750 & 404734 & 734242 & Upper glacial \\
\hline
\end{tabular}


Table 2A. Location and aquifer screened of public-supply wells in Kings, Queens, and Nassau Counties, N.Y.--continued

\begin{tabular}{|c|c|c|c|c|c|c|c|}
\hline $\begin{array}{c}\text { Well } \\
\text { number }\end{array}$ & Latitude & Longitude & $\begin{array}{c}\text { Aquifer in } \\
\text { which well is } \\
\text { screened }\end{array}$ & $\begin{array}{c}\text { Well } \\
\text { number }\end{array}$ & Latitude & Longitude & $\begin{array}{l}\text { Aquifer in } \\
\text { which well is } \\
\text { screened }\end{array}$ \\
\hline N7751 & 404734 & 734242 & Upper glacial & N8250 & 404108 & 733716 & Magothy \\
\hline N7752 & 404736 & 734240 & Upper glacial & N8251 & 403958 & 734103 & Magothy \\
\hline N7753 & 404736 & 734239 & Upper glacial & N8253 & 404003 & 733334 & Magothy \\
\hline N7754 & 404737 & 734238 & Upper glacial & N8264 & 404150 & 733732 & Magothy \\
\hline N7755 & 404734 & 734239 & Upper glacial & N8279 & 404309 & 733026 & Magothy \\
\hline N7756 & 404735 & 734238 & Upper glacial & N8313 & 405108 & 734304 & Upper glacial \\
\hline N7757 & 404734 & 734239 & Upper glacial & N8321 & 404400 & 733147 & Magothy \\
\hline N7758 & 404734 & 734238 & Upper glacial & N8326 & 405116 & 733729 & Upper glacial \\
\hline $\mathrm{N} 7772$ & 405012 & 733102 & Magothy & ${ }^{2} \mathrm{~N} 8327$ & 405113 & 733726 & Upper glacial \\
\hline N7773 & 405010 & 733059 & Magothy & N8339 & 404318 & 734011 & Magothy \\
\hline N7776 & 403537 & 733940 & Lloyd & N8342 & 404642 & 734405 & Lloyd \\
\hline N7781 & 404751 & 733220 & Magothy & N8354 & 403521 & 733659 & Lloyd \\
\hline N7785 & 404526 & 733534 & Magothy & N8355 & 404829 & 733159 & Magothy \\
\hline N7796 & 403949 & 733417 & Magothy & N8409 & 404420 & 733939 & Magothy \\
\hline N7797 & 404308 & 733321 & Magothy & N8420 & 404030 & 734146 & Magothy \\
\hline${ }^{2}$ N7804 & 403938 & 734232 & Upper glacial & N8457 & 404457 & 733607 & Magothy \\
\hline N7831 & 404022 & 733713 & Magothy & N8474 & 404325 & 733630 & Magothy \\
\hline N7852 & 404410 & 732616 & Magothy & N8475 & 404325 & 733630 & Magothy \\
\hline N7855 & 404040 & 734036 & Magothy & N8480 & 404228 & 732933 & Magothy \\
\hline N7857 & 405100 & 733842 & Lloyd & N8497 & 404519 & 733429 & Magothy \\
\hline N7873 & 404811 & 733633 & Magothy & N8525 & 404445 & 733104 & Magothy \\
\hline N7892 & 404651 & 734009 & Magothy & N8526 & 404454 & 733203 & Magothy \\
\hline N7957 & 404420 & 733531 & Magothy & N8557 & 403536 & 733948 & Lloyd \\
\hline N7961 & 404736 & 734242 & Upper glacial & N8558 & 404631 & 733832 & Magothy \\
\hline${ }^{2} \mathrm{~N} 7962$ & 404735 & 734236 & Upper glacial & N8576 & 404453 & 733834 & Magothy \\
\hline N7963 & 404734 & 734237 & Upper glacial & N8595 & 404554 & 732703 & Magothy \\
\hline N7964 & 404732 & 734237 & Upper glacial & N8603 & 404051 & 732603 & Magothy \\
\hline N7965 & 404733 & 734243 & Upper glacial & N8657 & 403925 & 733547 & Magothy \\
\hline N7966 & 404733 & 734240 & Upper glacial & N8658 & 404819 & 733433 & Magothy \\
\hline${ }^{2}$ N7968 & 404731 & 734236 & Upper glacial & N8664 & 404217 & 732904 & Magothy \\
\hline${ }^{2}$ N7971 & 404729 & 734236 & Upper glacial & N8665 & 404217 & 732901 & Magothy \\
\hline N8004 & 404344 & 732840 & Magothy & ${ }^{2} \mathrm{~N} 8672$ & 404133 & 733114 & Magothy \\
\hline N8007 & 404543 & 733549 & Magothy & N8713 & 404920 & 733733 & Magothy \\
\hline N8010 & 404739 & 733921 & Magothy & N8767 & 404532 & 732849 & Magothy \\
\hline N8011 & 403533 & 734013 & Lloyd & N8768 & 404533 & 732848 & Magothy \\
\hline N8031 & 404046 & 733058 & Magothy & N8776 & 405423 & 733353 & Lloyd \\
\hline N8043 & 404754 & 732831 & Magothy & N8778 & 404540 & 733043 & Magothy \\
\hline N8054 & 404557 & 732705 & Magothy & N8779 & 404539 & 733042 & Magothy \\
\hline N8183 & 405146 & 733134 & Upper glacial & N8818 & 404213 & 734100 & Magothy \\
\hline N8195 & 404233 & 734106 & Magothy & N8837 & 404050 & 732948 & Magothy \\
\hline N8196 & 403952 & 733616 & Magothy & N8941 & 404353 & 732910 & Magothy \\
\hline N8214 & 404156 & 732620 & Magothy & N8956 & 404508 & 733336 & Magothy \\
\hline N8216 & 404000 & 733710 & Magothy & N8957 & 404507 & 733337 & Magothy \\
\hline N8217 & 404004 & 733710 & Magothy & N8976 & 404119 & 733231 & Magothy \\
\hline N8218 & 404104 & 733743 & Magothy & N8979 & 404233 & 734106 & Magothy \\
\hline N8233 & 403519 & 733820 & Lloyd & ${ }^{2} \mathrm{~N} 9066$ & 405204 & 733634 & Magothy \\
\hline N8248 & 404525 & 733825 & Magothy & N9151 & 404224 & 734238 & Magothy \\
\hline N8249 & 404632 & 733111 & Magothy & ${ }^{2} \mathrm{~N} 9171$ & 404446 & 733813 & Upper glacial \\
\hline
\end{tabular}


Table 2A. Location and aquifer screened of public-supply wells in Kings, Queens, and Nassau Counties, N.Y.--continued

\begin{tabular}{|c|c|c|c|c|c|c|c|}
\hline $\begin{array}{c}\text { Well } \\
\text { number }\end{array}$ & Latitude & Longitude & $\begin{array}{l}\text { Aquifer in } \\
\text { which well is } \\
\text { screened }\end{array}$ & $\begin{array}{c}\text { Well } \\
\text { number }\end{array}$ & Latitude & Longitude & $\begin{array}{l}\text { Aquifer in } \\
\text { which well is } \\
\text { screened }\end{array}$ \\
\hline N9173 & 404154 & 732620 & Magothy & N9878 & 404117 & 733230 & Magothy \\
\hline N9180 & 404517 & 733102 & Magothy & N9910 & 404052 & 732948 & Magothy \\
\hline N9210 & 405202 & 733633 & Magothy & N9976 & 404059 & 733417 & Magothy \\
\hline N9211 & 405205 & 733634 & Magothy & N10033 & 404259 & 733806 & Magothy \\
\hline N9212 & 404453 & 733246 & Magothy & ${ }^{2} \mathrm{~N} 10034$ & 404258 & 733806 & Magothy \\
\hline${ }^{2} \mathrm{~N} 9277$ & 404256 & 733715 & Upper glacial & ${ }^{2} \mathrm{~N} 10103$ & 404023 & 733713 & Magothy \\
\hline${ }^{2} \mathrm{~N} 9285$ & 404002 & 733332 & Upper glacial & N10144 & 405421 & 733239 & Lloyd \\
\hline${ }^{2} \mathrm{~N} 9289$ & 403930 & 733828 & Upper glacial & ${ }^{2}$ N10149 & 405018 & 732845 & Magothy \\
\hline${ }^{2} \mathrm{~N} 9300$ & 405203 & 733227 & Upper glacial & ${ }^{2} \mathrm{~N} 10195$ & 404130 & 733114 & Magothy \\
\hline N9308 & 404735 & 734240 & Lloyd & ${ }^{2} \mathrm{~N} 10206$ & 404214 & 734241 & Magothy \\
\hline${ }^{2}$ N9323 & 405010 & 734149 & Upper glacial & ${ }^{2} \mathrm{~N} 10207$ & 404224 & 734240 & Magothy \\
\hline${ }^{2} \mathrm{~N} 9330$ & 405010 & 734148 & Port Washington & ${ }^{2} \mathrm{~N} 10208$ & 404537 & 733046 & Magothy \\
\hline N9334 & 405128 & 733705 & Lloyd & ${ }^{2} \mathrm{~N} 10211$ & 404125 & 734209 & Magothy \\
\hline N9338 & 404228 & 732935 & Magothy & ${ }^{2} \mathrm{~N} 10286$ & 404031 & 734144 & Magothy \\
\hline N9446 & 405127 & 734210 & Port Washington & ${ }^{2} \mathrm{~N} 10401$ & 404140 & 733841 & Magothy \\
\hline N9452 & 404137 & 733834 & Magothy & ${ }^{2} \mathrm{~N} 10408$ & 404140 & 733841 & Magothy \\
\hline N9463 & 404601 & 733150 & Magothy & ${ }^{2} \mathrm{~N} 10451$ & 404548 & 733609 & Magothy \\
\hline N9488 & 404628 & 733112 & Magothy & ${ }^{2} \mathrm{~N} 10555$ & 404609 & 733010 & Magothy \\
\hline N9514 & 404131 & 733114 & Magothy & ${ }^{2} \mathrm{~N} 10557$ & 404632 & 734017 & Magothy \\
\hline N9520 & 405144 & 733135 & Port Washington & ${ }^{2} \mathrm{~N} 10612$ & 404539 & 734014 & Magothy \\
\hline N9521 & 404411 & 733610 & Magothy & ${ }^{2} \mathrm{~N} 10863$ & 404056 & 732611 & Magothy \\
\hline N9591 & 404524 & 732826 & Magothy & ${ }^{2} \mathrm{~N} 10889$ & 404635 & 734011 & Magothy \\
\hline N9613 & 404033 & 734312 & Magothy & ${ }^{2} \mathrm{~N} 11004$ & 404408 & 732710 & Magothy \\
\hline N9768 & 404547 & 734011 & Magothy & ${ }^{2} \mathrm{~N} 11037$ & 404220 & 734244 & Magothy \\
\hline N9792 & 403932 & 733827 & Magothy & ${ }^{2} \mathrm{~N} 11076$ & 404908 & 733158 & Magothy \\
\hline N9809 & 404838 & 734042 & Upper glacial & ${ }^{2} \mathrm{~N} 11295$ & 404912 & 733156 & -. \\
\hline N9846 & 404412 & 733510 & Magothy & ${ }^{2} \mathrm{~N} 11647$ & 404125 & 734210 & Magothy \\
\hline
\end{tabular}


Table 2B. Public-supply pumpage in Kings, Queens, and Nassau Counties, N.Y., 1898-1984 [Pumpage in thousands of gallons]

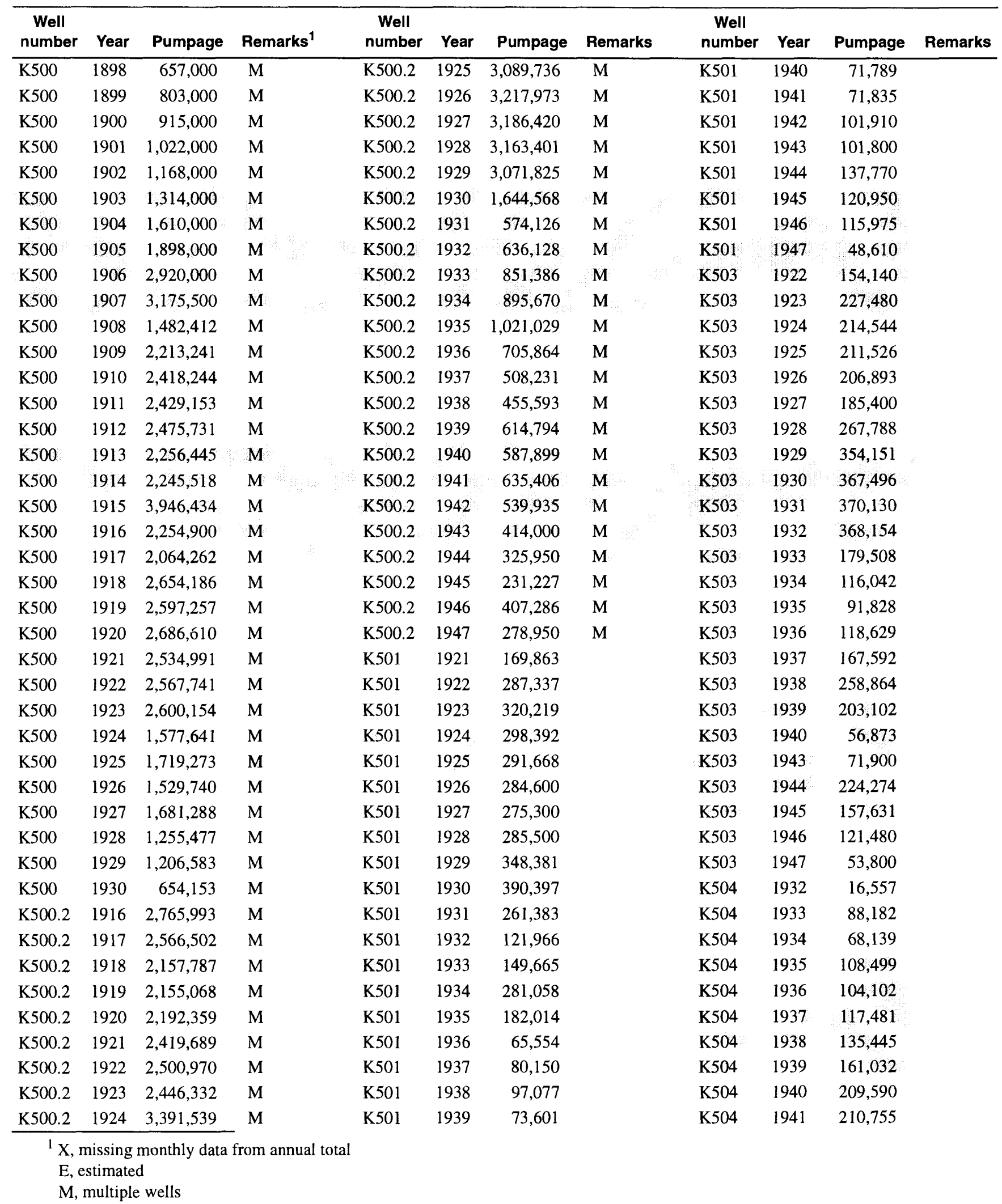


Table 2B. Public-supply pumpage in Kings, Queens, and Nassau Counties, N.Y., 1898-1984--continued

\begin{tabular}{|c|c|c|c|c|c|c|c|c|c|c|}
\hline $\begin{array}{c}\text { Well } \\
\text { number }\end{array}$ & Year & Pumpage Remarks & $\begin{array}{c}\text { Well } \\
\text { number }\end{array}$ & Year & Pumpage & Remarks & $\begin{array}{c}\text { Well } \\
\text { number }\end{array}$ & Year & Pumpage & Remarks \\
\hline K504 & 1942 & 211,582 & K507 & 1935 & 276,687 & & K510 & 1932 & 475,001 & \\
\hline K504 & 1943 & 266,000 & K507 & 1936 & 379,910 & & K510 & 1933 & 514,490 & \\
\hline K504 & 1944 & 239,413 & K507 & 1937 & 321,046 & & K510 & 1934 & 519,305 & \\
\hline K504 & 1945 & 220,752 & K507 & 1938 & 378,876 & & K510 & 1935 & 469,446 & \\
\hline K504 & 1946 & 355,707 & K507 & 1939 & 430,170 & & K510 & 1936 & 512,864 & \\
\hline K504 & 1947 & 265,637 & K507 & 1940 & 421,240 & & K510 & 1937 & 525,286 & \\
\hline K505 & 1944 & $1,090,700$ & K507 & 1941 & 396,160 & & K510 & 1938 & 536,210 & \\
\hline K505 & 1945 & $1,038,526$ & K507 & 1942 & 382,265 & & K510 & 1939 & 549,495 & \\
\hline K505 & 1946 & $1,237,406$ & K507 & 1943 & 383,000 & & K510 & 1940 & 478,000 & \\
\hline K505 & 1947 & 678,523 & K507 & 1944 & 313,470 & & K510 & 1941 & 451,150 & \\
\hline K506 & 1923 & 141,534 & K507 & 1945 & 303,035 & & K510 & 1942 & 366,055 & \\
\hline K506 & 1924 & 315,089 & K507 & 1946 & 300,365 & & K510 & 1943 & 265,000 & \\
\hline K506 & 1925 & 301,739 & K507 & 1947 & 173,310 & & K510 & 1944 & 257,230 & \\
\hline K506 & 1926 & 299,870 & K508 & 1924 & 252,056 & & K510 & 1945 & 270,500 & \\
\hline K506 & 1927 & 290,921 & K508 & 1925 & 320,594 & & K510 & 1946 & 302,250 & \\
\hline K506 & 1928 & 365,900 & K508 & 1926 & 323,900 & & K5 10 & 1947 & 110,960 & \\
\hline K506 & 1929 & 356,939 & K508 & 1927 & 314,200 & & K512 & 1925 & 185,372 & \\
\hline K506 & 1930 & 316,961 & K508 & 1928 & 344,500 & & K512 & 1926 & 358,928 & \\
\hline K506 & 1931 & 255,723 & K508 & 1929 & 421,173 & & $\mathrm{~K} 512$ & 1927 & 365,200 & \\
\hline K506 & 1932 & 185,139 & K508 & 1930 & 470,223 & & K512 & 1928 & 377,760 & \\
\hline K506 & 1933 & 296,322 & K508 & 1931 & 417,248 & & K512 & 1929 & 424,279 & \\
\hline K506 & 1934 & 389,276 & K508 & 1932 & 362,585 & & K512 & 1930 & 488,887 & \\
\hline K506 & 1935 & 456,337 & K508 & 1933 & 440,989 & & K512 & 1931 & 409,196 & \\
\hline K506 & 1936 & 459,138 & K508 & 1934 & 482,543 & & K512 & 1932 & 460,615 & \\
\hline K506 & 1937 & 503,103 & K508 & 1935 & 436,906 & & K512 & 1933 & 435,606 & \\
\hline K506 & 1938 & 478,825 & K508 & 1936 & 583,688 & & K512 & 1934 & 469,914 & \\
\hline K506 & 1939 & 509,104 & K508 & 1937 & 586,242 & & K512 & 1935 & 479,286 & \\
\hline K506 & 1940 & 457,204 & K508 & 1938 & 596,532 & & K512 & 1936 & 454,426 & \\
\hline K506 & 1941 & 375,225 & K508 & 1939 & 625,610 & & K512 & 1937 & 452,289 & \\
\hline K506 & 1942 & 346,080 & K508 & 1940 & 618,710 & & K512 & 1938 & 462,600 & \\
\hline K506 & 1943 & 342,000 & K508 & 1941 & 626,830 & & K512 & 1939 & 460,480 & \\
\hline K506 & 1944 & 334,390 & K508 & 1942 & 551,104 & & K512 & 1940 & 496,725 & \\
\hline K506 & 1945 & 327,085 & K508 & 1943 & 416,000 & & K512 & 1941 & 324,028 & \\
\hline K506 & 1946 & 327,800 & K508 & 1944 & 315,325 & & K512 & 1942 & 190,552 & \\
\hline K506 & 1947 & 152,980 & K508 & 1945 & 178,390 & & K512 & 1943 & 189,000 & \\
\hline K507 & 1925 & 198,171 & K508 & 1946 & 212,210 & & K512 & 1944 & 182,357 & \\
\hline K507 & 1926 & 250,040 & K508 & 1947 & 72,030 & & K512 & 1945 & 168,540 & \\
\hline K507 & 1927 & 98,740 & K510 & 1924 & 125,700 & & K512 & 1946 & 211,480 & \\
\hline K507 & 1928 & 263,600 & K510 & 1925 & 356,620 & & K512 & 1947 & 89,780 & \\
\hline K507 & 1929 & 197,580 & K510 & 1926 & 337,960 & & K513 & 1925 & 32,447 & \\
\hline K507 & 1930 & 163,415 & K510 & 1927 & 345,955 & & K513 & 1926 & 247,457 & \\
\hline K507 & 1931 & 153,790 & K510 & 1928 & 320,500 & & K513 & 1927 & 343,500 & \\
\hline K507 & 1932 & 124,393 & K510 & 1929 & 404,087 & & K513 & 1928 & 245,140 & \\
\hline K507 & 1933 & 136,251 & K510 & 1930 & 510,078 & & K513 & 1929 & 291,602 & \\
\hline K507 & 1934 & 294,018 & K510 & 1931 & 483,152 & & K513 & 1930 & 364,768 & \\
\hline
\end{tabular}


Table 2B. Public-supply pumpage in Kings, Queens, and Nassau Counties, N.Y., 1898-1984--continued

\begin{tabular}{|c|c|c|c|c|c|c|c|c|c|c|c|}
\hline $\begin{array}{c}\text { Well } \\
\text { number }\end{array}$ & Year & Pumpage & Remarks & $\begin{array}{c}\text { Well } \\
\text { number }\end{array}$ & Year & Pumpage & Remarks & $\begin{array}{c}\text { Well } \\
\text { number }\end{array}$ & Year & Pumpage & Remarks \\
\hline K513 & 1931 & 306,152 & & K516 & 1932 & 127,003 & & K519 & 1937 & 411,987 & \\
\hline K513 & 1932 & 258,433 & & K516 & 1933 & 201,941 & & K519 & 1938 & 358,262 & \\
\hline K513 & 1933 & 87,530 & & K516 & 1934 & 331,237 & & K519 & 1939 & 237,299 & \\
\hline K513 & 1934 & 122,523 & & K516 & 1935 & 329,771 & & K519 & 1940 & 214,964 & \\
\hline K513 & 1935 & 110,217 & & K516 & 1936 & 426,593 & & K519 & 1941 & 223,680 & \\
\hline K513 & 1936 & 56,928 & & K516 & 1937 & 485,145 & & K519 & 1942 & 201,750 & \\
\hline K513 & 1937 & 55,919 & & K516 & 1938 & 497,196 & & K519 & 1943 & 211,000 & \\
\hline K513 & 1938 & 60,141 & & K516 & 1939 & 492,210 & & К519 & 1944 & 198,025 & \\
\hline K513 & 1939 & 60,942 & & K516 & 1940 & 489,995 & & K519 & 1945 & 258,865 & \\
\hline K513 & 1940 & 64,994 & & K516 & 1941 & 488,095 & & K519 & 1946 & 372,530 & \\
\hline K513 & 1941 & 71,163 & & K516 & 1942 & 453,580 & & K519 & 1947 & 167,060 & \\
\hline K513 & 1942 & 56,268 & & K516 & 1943 & 456,000 & & K520 & 1929 & 139,164 & \\
\hline K513 & 1943 & 104,000 & & K516 & 1944 & 414,090 & & K520 & 1930 & 743,824 & \\
\hline K513 & 1944 & 104,510 & & K516 & 1945 & 381,285 & & K520 & 1931 & 561,029 & \\
\hline K513 & 1945 & 117,649 & & K516 & 1946 & 374,315 & & K520 & 1932 & 912,617 & \\
\hline K513 & 1946 & 150,351 & & K516 & 1947 & 195,120 & & K520 & 1933 & 855,376 & \\
\hline K513 & 1947 & 61,510 & & K517 & 1927 & 271,386 & & K520 & 1934 & 770,069 & \\
\hline K514 & 1926 & 169,800 & & K517 & 1928 & 303,167 & & K520 & 1935 & 847,902 & \\
\hline K514 & 1927 & 253,200 & & K517 & 1929 & 365,061 & & K520 & 1936 & 854,471 & \\
\hline K514 & 1928 & 331,900 & & K517 & 1930 & 349,642 & & K520 & 1937 & 847,329 & \\
\hline K514 & 1929 & 315,825 & & K517 & 1931 & 283,368 & & K520 & 1938 & 855,013 & \\
\hline K514 & 1930 & 333,057 & & K517 & 1932 & 210,132 & & K520 & 1939 & 833,010 & \\
\hline K514 & 1931 & 221,200 & & K517 & 1933 & 397,532 & & K520 & 1940 & 819,400 & \\
\hline K514 & 1932 & 95,528 & & K517 & 1934 & 397,803 & & K520 & 1941 & 796,420 & \\
\hline K514 & 1933 & 63,962 & & K517 & 1935 & 369,113 & & K520 & 1942 & 769,230 & \\
\hline K514 & 1934 & 73,173 & & K517 & 1936 & 397,239 & & K520 & 1943 & 623,000 & \\
\hline K514 & 1935 & 3,936 & & K517 & 1937 & 398,241 & & K520 & 1944 & 495,700 & \\
\hline K514 & 1936 & 52,988 & & K517 & 1938 & 395,569 & & K520 & 1945 & 247,875 & \\
\hline K514 & 1937 & 58,211 & & K517 & 1939 & 386,815 & & K520 & 1946 & 5,030 & \\
\hline K514 & 1938 & 49,924 & & K517 & 1940 & 475,505 & & K521 & 1929 & 190,936 & \\
\hline K514 & 1939 & 93,181 & & K517 & 1941 & 497,276 & & K521 & 1930 & 932,728 & \\
\hline K514 & 1940 & 94,494 & & K517 & 1942 & 481,950 & & K521 & 1931 & $1,104,541$ & \\
\hline K514 & 1941 & 134,475 & & K517 & 1943 & 473,000 & & K521 & 1932 & 567,014 & \\
\hline K514 & 1942 & 122,030 & & K517 & 1944 & 460,270 & & K521 & 1933 & 300,791 & \\
\hline K514 & 1943 & 135,000 & & K517 & 1945 & 449,570 & & K521 & 1934 & 213,635 & \\
\hline K514 & 1944 & 91,380 & & K517 & 1946 & 451,150 & & K521 & 1935 & 118,349 & \\
\hline K514 & 1945 & 120,540 & & K517 & 1947 & 213,440 & & K521 & 1936 & 118,729 & \\
\hline K514 & 1946 & 185,650 & & K519 & 1929 & 482,666 & & $\mathrm{~K} 521$ & 1937 & 138,218 & \\
\hline K514 & 1947 & 53,895 & & K519 & 1930 & 679,322 & & K521 & 1938 & 178,173 & \\
\hline K516 & 1926 & 103,625 & & K519 & 1931 & 656,455 & & K521 & 1939 & 279,681 & \\
\hline K516 & 1927 & 350,320 & & K519 & 1932 & 424,230 & & K521 & 1940 & 197,043 & \\
\hline K516 & 1928 & 394,300 & & K519 & 1933 & 758,579 & & K521 & 1941 & 205,005 & \\
\hline K516 & 1929 & 353,071 & & K519 & 1934 & 643,857 & & K521 & 1942 & 52,330 & \\
\hline K516 & 1930 & 218,593 & & K519 & 1935 & 583,034 & & K521 & 1943 & 101,000 & \\
\hline K516 & 1931 & 342,602 & & K519 & 1936 & 434,999 & & K521 & 1944 & 86,610 & \\
\hline
\end{tabular}


Table 2B. Public-supply pumpage in Kings, Queens, and Nassau Counties, N.Y., 1898-1984--continued

\begin{tabular}{|c|c|c|c|c|c|c|c|c|c|c|c|}
\hline $\begin{array}{c}\text { Well } \\
\text { number }\end{array}$ & Year & Pumpage & Remarks & $\begin{array}{c}\text { Well } \\
\text { number }\end{array}$ & Year & Pumpage & Remarks & $\begin{array}{c}\text { Well } \\
\text { number }\end{array}$ & Year & Pumpage & Remarks \\
\hline K521 & 1945 & 57,165 & & K525 & 1932 & 345,485 & & K528 & 1941 & 810,835 & \\
\hline K521 & 1946 & 10,520 & & K525 & 1933 & 82,322 & & K528 & 1942 & 744,400 & \\
\hline K522 & 1930 & 319,077 & & K525 & 1934 & 36,630 & & K528 & 1943 & 376,000 & \\
\hline K522 & 1931 & 991,447 & & K525 & 1935 & 13,423 & & K528 & 1944 & 8,500 & \\
\hline K522 & 1932 & $1,051,775$ & & K525 & 1936 & 144,839 & & K529 & 1934 & 39,461 & \\
\hline K522 & 1933 & 864,647 & & K525 & 1937 & 118,619 & & K529 & 1935 & 245,681 & \\
\hline K522 & 1934 & 879,083 & & K525 & 1938 & 2,470 & & K529 & 1936 & 278,083 & \\
\hline K522 & 1935 & 849,974 & & K526 & 1931 & 139,041 & & K529 & 1937 & 283,405 & \\
\hline K522 & 1936 & 905,241 & & K526 & 1932 & $1,107,477$ & & K529 & 1938 & 294,060 & \\
\hline K522 & 1937 & 933,551 & & K526 & 1933 & $1,040,654$ & & K529 & 1939 & 303,585 & \\
\hline K522 & 1938 & 926,058 & & K526 & 1934 & 826,462 & & K529 & 1940 & 212,390 & \\
\hline K522 & 1939 & 727,485 & & K526 & 1935 & 544,867 & & K529 & 1941 & 238,260 & \\
\hline K522 & 1940 & 534,475 & & K526 & 1936 & 544,748 & & K529 & 1942 & 178,805 & \\
\hline K522 & 1941 & 487,070 & & K526 & 1937 & 155,642 & & K529 & 1943 & 139,000 & \\
\hline K522 & 1942 & 424,530 & & K526 & 1938 & 65,886 & & K530 & 1935 & 515,882 & \\
\hline K522 & 1943 & 323,000 & & K526 & 1939 & 295,439 & & K530 & 1936 & 741,726 & \\
\hline K522 & 1944 & 338,450 & & K526 & 1940 & 392,726 & & K530 & 1937 & 731,704 & \\
\hline K522 & 1945 & 330,410 & & K526 & • 1941 & 351,895 & & K530 & 1938 & 452,787 & \\
\hline K522 & 1946 & 367,530 & & K526 & 1942 & 375,382 & & K530 & 1939 & 228,932 & \\
\hline K522 & 1947 & 176,080 & & K526 & 1943 & 373,230 & $\mathrm{E}$ & K530 & 1940 & 119,759 & \\
\hline K523 & 1930 & 305,939 & & K526 & 1944 & 382,239 & & K530 & 1941 & 108,640 & \\
\hline K523 & 1931 & 554,960 & & K526 & 1945 & 361,340 & $\mathrm{E}$ & K530 & 1942 & 113,170 & \\
\hline K523 & 1932 & 501,039 & & K526 & 1946 & 319,582 & $\mathrm{E}$ & K530 & 1943 & 163,000 & \\
\hline K523 & 1933 & 764,097 & & K526 & 1947 & 258,989 & $\mathrm{E}$ & K530 & 1944 & 149,210 & \\
\hline K523 & 1934 & 233,236 & & K527 & 1934 & 68,742 & & K530 & 1945 & 114,426 & \\
\hline K523 & 1935 & 5,698 & & K527 & 1935 & 185,797 & & K530 & 1946 & 109,374 & \\
\hline K523 & 1936 & 93,102 & & K527 & 1936 & 232,291 & & K530 & 1947 & 28,750 & \\
\hline K524 & 1931 & 507,594 & & K527 & 1937 & 292,689 & & K531 & 1937 & 708,206 & \\
\hline K524 & 1932 & 837,256 & & K527 & 1938 & 291,592 & & K531 & 1938 & 915,383 & \\
\hline K524 & 1933 & 679,843 & & K527 & 1939 & 311,805 & & K531 & 1939 & 819,945 & \\
\hline K524 & 1934 & 524,278 & & K527 & 1940 & 281,320 & & K531 & 1940 & 712,824 & \\
\hline K524 & 1935 & 548,275 & & K527 & 1941 & 288,755 & & K531 & 1941 & 737,097 & \\
\hline K524 & 1936 & 528,181 & & K527 & 1942 & 315,785 & & K531 & 1942 & 750,778 & \\
\hline K524 & 1937 & 464,682 & & K527 & 1943 & 316,000 & & K531 & 1943 & 757,770 & $\mathrm{E}$ \\
\hline K524 & 1938 & 504,252 & & K527 & 1944 & 324,130 & & K531 & 1944 & 774,701 & \\
\hline K524 & 1939 & 402,180 & & K527 & 1945 & 295,670 & & K531 & 1945 & 733,630 & $\mathrm{E}$ \\
\hline K524 & 1940 & 392,635 & & K527 & 1946 & 290,490 & & K531 & 1946 & 648,848 & $\mathrm{E}$ \\
\hline K524 & 1941 & 392,375 & & K527 & 1947 & 144,810 & & K531 & 1947 & 127,561 & $\mathrm{E}$ \\
\hline K524 & 1942 & 571,220 & & K528 & 1934 & 243,288 & & K1232 & 1941 & 126,273 & $\mathrm{E}$ \\
\hline K524 & 1943 & 546,000 & & K528 & 1935 & 763,106 & & K1232 & 1942 & 252,818 & $\mathrm{E}$ \\
\hline K524 & 1944 & 455,520 & & К528 & 1936 & 844,176 & & K1232 & 1943 & 246,666 & $\mathrm{E}$ \\
\hline K524 & 1945 & 360,525 & & K528 & 1937 & 856,432 & & K1232 & 1944 & 253,920 & $\mathrm{E}$ \\
\hline K524 & 1946 & 267,180 & & K528 & 1938 & 855,126 & & K1232 & 1945 & 253,730 & $\mathrm{E}$ \\
\hline K524 & 1947 & 136,310 & & K528 & 1939 & 778,750 & & K1232 & 1946 & 240,343 & $\mathrm{E}$ \\
\hline K525 & 1931 & 297,471 & & K528 & 1940 & 851,345 & & K1232 & 1947 & 117,556 & $\mathrm{E}$ \\
\hline
\end{tabular}


Table 2B. Public-supply pumpage in Kings, Queens, and Nassau Counties, N.Y., 1898-1984--continued

\begin{tabular}{|c|c|c|c|c|c|c|c|c|c|c|c|}
\hline $\begin{array}{c}\text { Well } \\
\text { number }\end{array}$ & Year & Pumpage & Remarks & $\begin{array}{c}\text { Well } \\
\text { number }\end{array}$ & Year & Pumpage & Remarks & $\begin{array}{c}\text { Well } \\
\text { number }\end{array}$ & Year & Pumpage & Remarks \\
\hline K1233 & 1941 & 126,273 & $\mathrm{E}$ & K1359 & 1946 & 296,500 & & Q301 & 1924 & 291,277 & \\
\hline K1233 & 1942 & 252,818 & $\mathrm{E}$ & K1359 & 1947 & 146,510 & & Q301 & 1925 & 240,178 & \\
\hline K1233 & 1943 & 246,666 & $\mathrm{E}$ & K1360 & 1944 & 233,440 & & Q301 & 1926 & 237,322 & \\
\hline K1233 & 1944 & 253,920 & $\mathrm{E}$ & K1360 & 1945 & 483,610 & & Q301 & 1927 & 240,389 & \\
\hline K1233 & 1945 & 253,730 & $\mathrm{E}$ & K1360 & 1946 & 494,340 & & Q301 & 1928 & 220,916 & \\
\hline K1233 & 1946 & 240,343 & $\mathrm{E}$ & K1360 & 1947 & 276,130 & & $\mathrm{Q} 301$ & 1929 & 197,018 & \\
\hline K1233 & 1947 & 117,556 & $\mathrm{E}$ & K1363 & 1945 & 166,640 & & Q301 & 1930 & 150,421 & \\
\hline K 1234 & 1941 & 126,274 & $\mathrm{E}$ & K1363 & 1946 & 259,540 & & Q301 & 1931 & 111,789 & \\
\hline K1234 & 1942 & 252,819 & $\mathrm{E}$ & K1363 & 1947 & 125,880 & & Q301 & 1932 & 125,857 & \\
\hline K 1234 & 1943 & 246,668 & $\mathrm{E}$ & K1516 & 1946 & 69,670 & & Q301 & 1933 & 135,430 & \\
\hline K1234 & 1944 & 253,920 & $E$ & K1516 & 1947 & 120,440 & & Q301 & 1934 & 66,940 & \\
\hline K1234 & 1945 & 253,730 & $E$ & Q68 & 1940 & 143,036 & & Q301 & 1935 & 123,630 & \\
\hline K1234 & 1946 & 240,344 & $\mathrm{E}$ & Q68 & 1941 & 142,111 & & Q301 & 1936 & 138,491 & \\
\hline K1234 & 1947 & 117,558 & $\mathrm{E}$ & Q68 & 1942 & 142,800 & & Q301 & 1937 & 132,453 & \\
\hline K1329 & 1942 & 27,260 & & Q68 & 1943 & 144,000 & & Q301 & 1938 & 59,894 & \\
\hline K1329 & 1943 & 309,000 & & Q68 & 1944 & 317,450 & & Q301 & 1939 & 22,507 & \\
\hline K1329 & 1944 & 289,280 & & Q68 & 1945 & 534,735 & & Q301 & 1940 & 108,366 & \\
\hline K1329 & 1945 & 285,350 & & Q68 & 1946 & 531,594 & & Q301 & 1941 & 59,397 & \\
\hline K1329 & 1946 & 276,390 & & Q68 & 1947 & 514,561 & & Q301 & 1942 & 8,333 & \\
\hline K1329 & 1947 & 130,290 & & Q68 & 1948 & 471,652 & & Q301 & 1943 & 26,028 & \\
\hline K1331 & 1943 & 261,070 & & Q68 & 1949 & 233,314 & & Q301 & 1944 & 24,134 & \\
\hline K1331 & 1944 & 287,620 & & Q68 & 1950 & 209,875 & & Q301 & 1974 & 6,400 & \\
\hline K1331 & 1945 & 286,880 & & Q68 & 1951 & 206,225 & & Q301 & 1975 & 3,900 & \\
\hline K1331 & 1946 & 272,940 & & Q68 & 1952 & 256,960 & & Q301 & 1976 & 17,000 & \\
\hline K1331 & 1947 & 125,975 & & Q68 & 1953 & 198,380 & & Q301 & 1977 & 21,400 & \\
\hline K1338 & 1943 & 241,000 & & Q68 & 1954 & 192,946 & & Q301 & 1978 & 28,700 & \\
\hline K1338 & 1944 & 303,197 & & Q68 & 1955 & 123,510 & & Q301 & 1979 & 39,900 & \\
\hline K1338 & 1945 & 297,505 & & Q68 & 1956 & 38,412 & & Q301 & 1980 & 30,600 & \\
\hline K1338 & 1946 & 298,730 & & Q68 & 1957 & 10,016 & & $\mathrm{Q} 301$ & 1981 & 50,700 & \\
\hline K1338 & 1947 & 144,030 & & Q68 & 1958 & 4,050 & & Q301 & 1982 & 58,900 & \\
\hline K1343 & 1943 & 157,000 & & Q68 & 1959 & 9,810 & & Q301 & 1983 & 62,600 & \\
\hline K1343 & 1944 & 294,280 & & Q68 & 1960 & 4,270 & & Q301 & 1984 & 33,300 & \\
\hline K1343 & 1945 & 285,035 & & Q68 & 1961 & 2,028 & & Q302 & 1921 & 12,000 & $E$ \\
\hline K1343 & 1946 & 280,040 & & Q68 & 1962 & 14,220 & & Q302 & 1922 & 24,000 & $\mathrm{E}$ \\
\hline K1343 & 1947 & 134,320 & & Q68 & 1963 & 16,905 & & Q302 & 1923 & 21,000 & $\mathrm{E}$ \\
\hline K1351 & 1944 & 153,761 & & Q68 & 1964 & 18,090 & & Q302 & 1924 & 20,080 & \\
\hline K1351 & 1945 & 155,421 & & Q68 & 1965 & 1,430 & & Q302 & 1925 & 9,665 & \\
\hline $\mathrm{K} 1351$ & 1946 & 147,189 & & Q68 & 1966 & 24,376 & & Q302 & 1926 & 5,163 & \\
\hline K1351 & 1947 & 86,260 & & Q68 & 1967 & 10,260 & & Q302 & 1927 & 20,272 & \\
\hline K1357 & 1944 & 467,415 & & Q68 & 1968 & 11,550 & & Q302 & 1928 & 35,556 & \\
\hline K1357 & 1945 & 686,111 & & Q68 & 1969 & 6,760 & & Q302 & 1929 & 39,636 & \\
\hline K1357 & 1946 & 693,171 & & Q68 & 1970 & 6,760 & & Q302 & 1930 & 20,382 & \\
\hline K1357 & 1947 & 336,450 & & Q68 & 1971 & 18,778 & & Q302 & 1931 & 8,320 & \\
\hline K1359 & 1944 & 145,540 & & Q68 & 1972 & 984 & & $\mathrm{Q} 302$ & 1932 & 16,127 & \\
\hline K1359 & 1945 & 296,375 & & Q301 & 1923 & 316,790 & & Q302 & 1933 & 17,261 & \\
\hline
\end{tabular}


Table 2B. Public-supply pumpage in Kings, Queens, and Nassau Counties, N.Y., 1898-1984--continued

\begin{tabular}{|c|c|c|c|c|c|c|c|c|c|c|c|}
\hline $\begin{array}{c}\text { Well } \\
\text { number }\end{array}$ & Year & Pumpage & Remarks & $\begin{array}{c}\text { Well } \\
\text { number }\end{array}$ & Year & Pumpage & Remarks & $\begin{array}{c}\text { Well } \\
\text { number }\end{array}$ & Year & Pumpage & Remarks \\
\hline Q302 & 1934 & 8,120 & & Q304 & 1926 & 137,675 & & Q305 & 1939 & 94,899 & \\
\hline Q302 & 1935 & 7,235 & & Q304 & 1927 & 180,000 & & Q305 & 1940 & 83,131 & \\
\hline Q302 & 1936 & 4,990 & & Q304 & 1928 & 150,000 & & Q305 & 1941 & 96,477 & \\
\hline Q302 & 1937 & 1,737 & & Q304 & 1929 & 86,661 & & Q305 & 1942 & 30,990 & \\
\hline Q302 & 1938 & 63 & & Q304 & 1930 & 17,940 & & Q305 & 1943 & 47,680 & \\
\hline Q302 & 1939 & 4,577 & & Q304 & 1931 & 42,757 & & Q305 & 1944 & 102,656 & \\
\hline Q302 & 1941 & 13,096 & & Q304 & 1932 & 43,083 & & Q305 & 1976 & 7,060 & \\
\hline Q302 & 1943 & 9,637 & & Q304 & 1933 & 41,428 & & Q306 & 1908 & 49,500 & M \\
\hline Q302 & 1944 & 39,010 & & Q304 & 1934 & 42,809 & & Q306 & 1910 & 30,545 & M \\
\hline Q303 & 1922 & 56,590 & & Q304 & 1935 & 18,118 & & Q306 & 1911 & 51,939 & M \\
\hline Q303 & 1923 & 238,580 & & Q304 & 1936 & 16,325 & & Q306 & 1913 & 238,952 & M \\
\hline Q303 & 1924 & 359,796 & & Q304 & 1937 & 15,819 & & Q306 & 1914 & 341,958 & M \\
\hline Q303 & 1925 & 370,349 & & Q304 & 1938 & 10,593 & & Q306 & 1915 & 468,291 & $\mathrm{M}$ \\
\hline Q303 & 1926 & 247,592 & & Q304 & 1939 & 10,188 & & Q306 & 1916 & 519,800 & $\mathrm{X}, \mathrm{M}$ \\
\hline Q303 & 1927 & 213,894 & & Q304 & 1940 & 20,286 & & Q306 & 1917 & 302,000 & M \\
\hline Q303 & 1928 & 164,380 & & Q304 & 1941 & 19,436 & & Q306 & 1918 & 456,610 & M \\
\hline Q303 & 1929 & 160,175 & & Q304 & 1942 & 10,167 & & Q306 & 1919 & 476,400 & M \\
\hline Q303 & 1930 & 211,130 & & Q304 & 1943 & 11,924 & & Q306 & 1920 & 484,570 & M \\
\hline Q303 & 1931 & 282,050 & & Q304 & 1944 & 213,860 & & Q306 & 1921 & 351,140 & $M$ \\
\hline Q303 & 1932 & 335,505 & & Q304 & 1974 & 55,930 & & Q306 & 1922 & 465,320 & M \\
\hline Q303 & 1933 & 269,024 & & Q304 & 1975 & 75,310 & & Q306 & 1923 & 541,160 & M \\
\hline Q303 & 1934 & 317,666 & & Q304 & 1976 & 32,790 & & Q306 & 1924 & 679,980 & M \\
\hline Q303 & 1935 & 389,610 & & Q304 & 1977 & 50,670 & & Q306 & 1925 & 594,600 & $\mathrm{M}$ \\
\hline Q303 & 1936 & 382,700 & & Q304 & 1978 & 40,500 & & Q306 & 1926 & 884,740 & $\mathrm{X}, \mathrm{M}$ \\
\hline Q303 & 1937 & 334,305 & & Q304 & 1979 & 61,740 & & Q306 & 1927 & $1,061,420$ & $\mathrm{M}$ \\
\hline Q303 & 1938 & 146,883 & & Q304 & 1980 & 67,930 & & Q306 & 1928 & 623,780 & M \\
\hline Q303 & 1939 & 194,238 & & Q304 & 1981 & 81,870 & & Q306 & 1929 & $1,039,200$ & $\mathrm{M}$ \\
\hline Q303 & 1940 & 122,421 & & Q304 & 1982 & 148,650 & & Q306 & 1930 & $1,229,850$ & $\mathrm{M}$ \\
\hline Q303 & 1941 & 158,685 & & Q304 & 1983 & 61,770 & & Q306 & 1931 & $1,043,610$ & M \\
\hline Q303 & 1942 & 83,840 & & Q304 & 1984 & 34,670 & & Q306 & 1932 & 852,140 & M \\
\hline Q303 & 1943 & 70,896 & & Q305 & 1924 & 117,726 & & Q306 & 1933 & 902,550 & $\mathbf{M}$ \\
\hline Q303 & 1944 & 214,547 & & Q305 & 1925 & 428,860 & & Q306 & 1934 & 540,430 & $\mathbf{M}$ \\
\hline Q303 & 1974 & 112,400 & & Q305 & 1926 & 391,957 & & Q306 & 1935 & 94,659 & $\mathrm{E}$ \\
\hline Q303 & 1975 & 76,000 & & Q305 & 1927 & 197,695 & & Q306 & 1936 & 272,220 & E \\
\hline Q303 & 1976 & 74,980 & & Q305 & 1928 & 349,252 & & Q306 & 1937 & 262,617 & $\mathrm{E}$ \\
\hline Q303 & 1977 & 196,720 & & Q305 & 1929 & 253,349 & & Q306 & 1938 & 261,835 & $\mathrm{E}$ \\
\hline Q303 & 1978 & 193,400 & & Q305 & 1930 & 311,652 & & Q306 & 1939 & 281,257 & $\mathrm{E}$ \\
\hline Q303 & 1979 & 195,500 & & Q305 & 1931 & 307,420 & & Q306 & 1940 & 283,912 & $\mathrm{E}$ \\
\hline Q303 & 1980 & 156,300 & & Q305 & 1932 & 283,512 & & Q306 & 1941 & 285,985 & $\mathrm{E}$ \\
\hline Q303 & 1981 & 218,000 & & Q305 & 1933 & 151,464 & & Q306 & 1942 & 272,025 & $\mathrm{E}$ \\
\hline Q303 & 1982 & 211,900 & & Q305 & 1934 & 100,230 & & Q306 & 1943 & 293,975 & $\mathrm{E}$ \\
\hline Q303 & 1983 & 82,800 & & Q305 & 1935 & 239,930 & & Q306 & 1944 & 289,530 & $\mathrm{E}$ \\
\hline Q303 & 1984 & 113,100 & & Q305 & 1936 & 192,765 & & Q307 & 1924 & 145,887 & \\
\hline Q304 & 1924 & 181,734 & & Q305 & 1937 & 98,333 & & Q307 & 1925 & 304,266 & \\
\hline Q304 & 1925 & 184,982 & & Q305 & 1938 & 53,588 & & Q307 & 1926 & 211,086 & \\
\hline
\end{tabular}


Table 2B. Public-supply pumpage in Kings, Queens, and Nassau Counties, N.Y., 1898-1984--continued

\begin{tabular}{|c|c|c|c|c|c|c|c|c|c|c|c|}
\hline $\begin{array}{c}\text { Well } \\
\text { number }\end{array}$ & Year & Pumpage & Remarks & $\begin{array}{c}\text { Well } \\
\text { number }\end{array}$ & Year & Pumpage & Remarks & $\begin{array}{c}\text { Well } \\
\text { number }\end{array}$ & Year & Pumpage & Remarks \\
\hline Q307 & 1927 & 275,066 & & Q308 & 1942 & 44,389 & & Q311 & 1926 & 229,515 & \\
\hline Q307 & 1928 & 361,478 & & Q308 & 1943 & 175,115 & & Q311 & 1927 & 305,006 & \\
\hline Q307 & 1929 & 487,035 & & Q308 & 1944 & 338,300 & & Q311 & 1928 & 374,060 & \\
\hline Q307 & 1930 & 301,712 & & Q308 & 1974 & 85,400 & & Q311 & 1929 & 315,328 & \\
\hline Q307 & 1931 & 233,128 & & Q308 & 1975 & 110,000 & & Q311 & 1930 & 172,955 & \\
\hline Q307 & 1932 & 361,712 & & Q308 & 1976 & 142,700 & & Q311 & 1931 & 114,691 & \\
\hline Q307 & 1933 & 350,705 & & Q308 & 1977 & 101,600 & & Q311 & 1932 & 87,475 & \\
\hline Q307 & 1934 & 311,666 & & Q308 & 1978 & 170,500 & & Q311 & 1933 & 284,216 & \\
\hline Q307 & 1935 & 298,803 & & Q308 & 1979 & 162,000 & & Q311 & 1934 & 249,739 & \\
\hline Q307 & 1936 & 300,257 & & Q308 & 1980 & 77,200 & & Q311 & 1935 & 269,668 & \\
\hline Q307 & 1937 & 295,883 & & Q308 & 1981 & 113,100 & & Q311 & 1936 & 72,444 & \\
\hline Q307 & 1938 & 303,205 & & Q308 & 1982 & 77,600 & & Q311 & 1937 & 161,701 & \\
\hline Q307 & 1939 & 483,067 & & Q308 & 1983 & 1,100 & & Q311 & 1938 & 59,825 & \\
\hline Q307 & 1940 & 433,425 & & Q310 & 1925 & 160,239 & & Q311 & 1939 & 22,108 & \\
\hline Q307 & 1941 & 361,537 & & Q310 & 1926 & 174,596 & & Q311 & 1941 & 118,480 & \\
\hline Q307 & 1942 & 402,118 & & Q310 & 1927 & 138,994 & & Q311 & 1942 & 324,511 & \\
\hline Q307 & 1943 & 424,795 & & Q310 & 1928 & 124,354 & & Q311 & 1943 & 219,740 & \\
\hline Q307 & 1944 & 392,620 & & Q310 & 1929 & 131,127 & & Q311 & 1944 & 385,515 & \\
\hline Q307 & 1974 & 34,950 & & Q310 & 1930 & 203,122 & & Q311 & 1974 & 439,600 & \\
\hline Q307 & 1975 & 35,050 & & Q310 & 1931 & 100,952 & & Q311 & 1975 & 474,300 & \\
\hline Q307 & 1976 & 59,300 & & Q310 & 1932 & 119,586 & & Q311 & 1976 & 475,200 & \\
\hline Q307 & 1977 & 45,100 & & Q310 & 1933 & 127,530 & & Q311 & 1977 & 474,500 & \\
\hline Q307 & 1978 & 17,850 & & Q310 & 1934 & 22,298 & & Q311 & 1978 & 474,400 & \\
\hline Q307 & 1980 & 25,650 & & Q310 & 1935 & 27,618 & & Q311 & 1979 & 433,100 & \\
\hline Q307 & 1981 & 21,000 & & Q310 & 1936 & 55,191 & & Q311 & 1980 & 310,400 & \\
\hline Q307 & 1982 & 26,400 & & Q310 & 1937 & 20,682 & & Q311 & 1981 & 473,000 & \\
\hline Q307 & 1983 & 18,600 & & Q310 & 1938 & 75,091 & & Q311 & 1982 & 467,700 & \\
\hline Q308 & 1924 & 335,739 & & Q310 & 1939 & 268,633 & & Q311 & 1983 & 392,200 & \\
\hline Q308 & 1925 & 400,831 & & Q310 & 1940 & 374,517 & & Q311 & 1984 & 115,300 & \\
\hline Q308 & 1926 & 418,198 & & Q310 & 1941 & 405,077 & & Q312 & 1926 & 53,988 & \\
\hline Q308 & 1927 & 411,458 & & Q310 & 1942 & 231,097 & & Q312 & 1927 & 97,992 & \\
\hline Q308 & 1928 & 400,000 & & Q310 & 1943 & 279,166 & & Q312 & 1928 & 157,812 & \\
\hline Q308 & 1929 & 399,313 & & Q310 & 1944 & 306,730 & & Q312 & 1929 & 177,383 & \\
\hline Q308 & 1930 & 484,415 & & Q310 & 1974 & 19,550 & & Q312 & 1930 & 225,223 & \\
\hline Q308 & 1931 & 294,315 & & Q310 & 1975 & 15,100 & & Q312 & 1931 & 188,472 & \\
\hline Q308 & 1932 & 418,011 & & Q310 & 1976 & 29,600 & & Q312 & 1932 & 67,788 & \\
\hline Q308 & 1933 & 320,481 & & $\mathrm{Q} 310$ & 1977 & 32,700 & & Q312 & 1933 & 19,548 & \\
\hline Q308 & 1934 & 403,870 & & Q310 & 1978 & 37,200 & & Q312 & 1934 & 11,342 & \\
\hline Q308 & 1935 & 363,283 & & Q310 & 1979 & 24,000 & & $\mathrm{Q} 312$ & 1935 & 9,379 & \\
\hline Q308 & 1936 & 367,295 & & Q310 & 1980 & 3,500 & & Q312 & 1936 & 11,623 & \\
\hline Q308 & 1937 & 295,922 & & Q310 & 1981 & 118,100 & & Q312 & 1937 & 12,900 & \\
\hline Q308 & 1938 & 119,731 & & Q310 & 1982 & 172,200 & & Q312 & 1938 & 1,873 & \\
\hline Q308 & 1939 & 404,667 & & Q310 & 1983 & 272,400 & & Q312 & 1939 & 44,814 & \\
\hline Q308 & 1940 & 178,065 & & Q310 & 1984 & 64,500 & & Q312 & 1940 & 14,603 & \\
\hline Q308 & 1941 & 91,331 & & Q311 & 1925 & 230,700 & & Q312 & 1941 & 53,415 & \\
\hline
\end{tabular}


Table 2B. Public-supply pumpage in Kings, Queens, and Nassau Counties, N.Y., 1898-1984--continued

\begin{tabular}{|c|c|c|c|c|c|c|c|c|c|c|c|}
\hline $\begin{array}{c}\text { Well } \\
\text { number }\end{array}$ & Year & Pumpage & Remarks & $\begin{array}{c}\text { Well } \\
\text { number }\end{array}$ & Year & Pumpage & Remarks & $\begin{array}{c}\text { Well } \\
\text { number }\end{array}$ & Year & Pumpage & Remarks \\
\hline Q312 & 1942 & 23,206 & & Q314 & 1941 & 267,675 & & Q318 & 1933 & 403,022 & \\
\hline Q312 & 1943 & 74,002 & & Q314 & 1942 & 139,705 & & Q318 & 1934 & 341,983 & \\
\hline Q312 & 1944 & 77,935 & & Q314 & 1943 & 284,372 & & Q318 & 1935 & 294,027 & \\
\hline Q313 & 1925 & 393 & & Q314 & 1944 & 204,028 & & Q318 & 1936 & 223,293 & \\
\hline Q313 & 1926 & 369,735 & & Q314 & 1974 & 300,200 & & Q318 & 1937 & 251,438 & \\
\hline Q313 & 1927 & 263,586 & & Q314 & 1975 & 472,400 & & Q318 & 1938 & 264,985 & \\
\hline Q313 & 1928 & 291,742 & & Q314 & 1976 & 436,700 & & Q318 & 1939 & 261,660 & \\
\hline Q313 & 1929 & 264,028 & & Q314 & 1977 & 310,800 & & Q318 & 1940 & 212,605 & \\
\hline Q313 & 1930 & 432,535 & & Q314 & 1978 & 369,300 & & Q318 & 1941 & 236,830 & \\
\hline Q313 & 1931 & 534,852 & & Q314 & 1979 & 400,800 & & Q318 & 1942 & 197,945 & \\
\hline Q313 & 1932 & 503,039 & & Q314 & 1980 & 260,300 & & Q318 & 1943 & 174,315 & \\
\hline Q313 & 1933 & 504,271 & & Q314 & 1981 & 251,800 & & Q318 & 1944 & 168,010 & \\
\hline Q313 & 1934 & 391,477 & & Q314 & 1982 & 214,300 & & Q319 & 1930 & 66,193 & \\
\hline Q313 & 1935 & 477,000 & & Q314 & 1983 & 476,400 & & Q319 & 1931 & 121,573 & \\
\hline Q313 & 1936 & 499,819 & & Q314 & 1984 & 731,400 & & Q319 & 1932 & 125,450 & \\
\hline Q313 & 1937 & 451,335 & 8 & Q317 & 1929 & 208,660 & & Q319 & 1933 & 209,789 & \\
\hline Q313 & 1938 & 322,222 & & Q317 & 1930 & 291,690 & & Q319 & 1934 & 254,143 & \\
\hline Q313 & 1939 & 479,500 & & Q317 & 1931 & 332,220 & & Q319 & 1935 & 254,331 & \\
\hline Q313 & 1940 & 399,635 & & Q317 & 1932 & 396,036 & & Q319 & 1936 & 256,264 & \\
\hline Q313 & 1941 & 332,085 & & Q317 & 1933 & 446,249 & & Q319 & 1937 & 99,823 & \\
\hline Q313 & 1942 & 249,485 & & Q317 & 1934 & 434,328 & & Q319 & 1938 & 21,450 & \\
\hline Q313 & 1943 & 324,710 & & Q317 & 1935 & 418,741 & & Q319 & 1939 & 72,429 & \\
\hline Q313 & 1944 & 278,065 & & Q317 & 1936 & 392,788 & & Q319 & 1940 & 66,646 & \\
\hline Q313 & 1974 & 26,450 & & Q317 & 1937 & 354,283 & & Q319 & 1941 & 39,095 & \\
\hline Q313 & 1975 & 17,060 & & Q317 & 1938 & 513,039 & & Q319 & 1942 & 66,447 & \\
\hline Q313 & 1976 & 17,090 & & Q317 & 1939 & 471,924 & & Q319 & 1943 & 26,330 & \\
\hline Q313 & 1977 & 22,370 & & Q317 & 1940 & 459,150 & & Q319 & 1944 & 36,875 & \\
\hline Q313 & 1980 & 23,230 & & Q317 & 1941 & 394,100 & & Q319 & 1975 & 4,300 & \\
\hline Q313 & 1983 & 40,700 & & Q317 & 1942 & 412,550 & & Q319 & 1976 & 17,000 & \\
\hline Q313 & 1984 & 24,800 & & Q317 & 1943 & 382,200 & & Q319 & 1977 & 40,200 & \\
\hline Q314 & 1926 & 43,314 & & Q317 & 1944 & 402,280 & & Q319 & 1978 & 32,840 & \\
\hline Q314 & 1927 & 164,124 & & Q317 & 1974 & 571,300 & & Q321 & 1931 & 4,616 & \\
\hline Q314 & 1928 & 244,894 & & Q317 & 1975 & 583,400 & & Q321 & 1932 & 195,803 & \\
\hline Q314 & 1929 & 194,642 & & Q317 & 1976 & 581,700 & & Q321 & 1933 & 356,410 & \\
\hline Q314 & 1930 & 206,861 & & Q317 & 1977 & 572,000 & & Q321 & 1934 & 447,557 & \\
\hline Q314 & 1931 & 163,300 & & Q317 & 1978 & 546,100 & & Q321 & 1935 & 382,816 & \\
\hline Q314 & 1932 & 190,315 & & Q317 & 1979 & 440,600 & & Q321 & 1936 & 390,356 & \\
\hline Q314 & 1933 & 262,489 & & Q317 & 1980 & 547,000 & & Q321 & 1937 & 405,370 & \\
\hline Q314 & 1934 & 306,193 & & Q317 & 1981 & 543,500 & & Q321 & 1938 & 434,734 & \\
\hline Q314 & 1935 & 204,636 & & Q317 & 1982 & 513,000 & & Q321 & 1939 & 415,484 & \\
\hline Q314 & 1936 & 330,807 & & Q317 & 1983 & 446,500 & & Q321 & 1940 & 61,063 & \\
\hline Q314 & 1937 & 299,091 & & Q317 & 1984 & 386,200 & & Q321 & 1941 & 99,820 & \\
\hline Q314 & 1938 & 190,800 & & Q318 & 1930 & 225,101 & & Q321 & 1942 & 274,424 & \\
\hline Q314 & 1939 & 93,410 & & Q318 & 1931 & 533,524 & & Q321 & 1943 & 317,405 & \\
\hline Q314 & 1940 & 32,455 & & Q318 & 1932 & 487,555 & & Q321 & 1944 & 355,067 & \\
\hline
\end{tabular}


Table 2B. Public-supply pumpage in Kings, Queens, and Nassau Counties, N.Y., 1898-1984--continued

\begin{tabular}{|c|c|c|c|c|c|c|c|c|c|c|c|}
\hline $\begin{array}{c}\text { Well } \\
\text { number }\end{array}$ & Year & Pumpage & Remarks & $\begin{array}{c}\text { Well } \\
\text { number }\end{array}$ & Year & Pumpage & Remarks & $\begin{array}{c}\text { Well } \\
\text { number }\end{array}$ & Year & Pumpage & Remarks \\
\hline Q321 & 1974 & 50,625 & & Q323 & 1944 & 559,485 & & Q324.2 & 1938 & 343,135 & $E$ \\
\hline Q321 & 1975 & 41,400 & & Q323 & 1974 & 1,000 & & Q324.2 & 1939 & 329,292 & $\mathrm{E}$ \\
\hline Q321 & 1976 & 46,350 & & Q323 & 1975 & 9,800 & & Q324.2 & 1940 & 296,195 & $\mathrm{E}$ \\
\hline Q321 & 1977 & 21,750 & & Q323 & 1976 & 24,470 & & Q324.2 & 1941 & 357,145 & E \\
\hline Q321 & 1978 & 23,325 & & Q323 & 1977 & 11,000 & & Q324.2 & 1942 & 335,365 & $E$ \\
\hline Q321 & 1979 & 13,500 & & Q323 & 1978 & 17,700 & & $\mathrm{Q} 324.2$ & 1943 & 312,448 & $\mathrm{E}$ \\
\hline Q321 & 1980 & 20,925 & & Q323 & 1979 & 1,800 & & Q324.2 & 1944 & 333,813 & $\mathrm{E}$ \\
\hline Q321 & 1981 & 22,800 & & Q323 & 1980 & 5,500 & & Q351 & 1925 & 8,839 & $\mathrm{E}$ \\
\hline Q321 & 1982 & 23,925 & & Q323 & 1981 & 7,500 & & Q351 & 1926 & 9,301 & \\
\hline Q321 & 1984 & 160,400 & & Q323 & 1982 & 45,800 & & Q351 & 1927 & 8,777 & \\
\hline Q322 & 1932 & 135,448 & & Q323 & 1983 & 32,900 & & Q351 & 1928 & 3,990 & \\
\hline Q322 & 1933 & 264,442 & & Q323 & 1984 & 13,730 & & Q351 & 1936 & 146,936 & \\
\hline Q322 & 1934 & 373,170 & & Q324 & 1906 & 582,740 & $\mathbf{M}$ & Q351 & 1937 & 272,954 & \\
\hline Q322 & 1935 & 368,990 & & Q324 & 1907 & $1,007,327$ & M & Q351 & 1938 & 294,284 & \\
\hline Q322 & 1936 & 356,338 & & Q324 & 1908 & $1,030,868$ & M & Q351 & 1939 & 442,054 & \\
\hline Q322 & 1937 & 367,799 & & Q324 & 1909 & $1,185,613$ & M & $\mathrm{Q} 351$ & 1940 & 417,442 & \\
\hline Q322 & 1938 & 339,532 & & Q324 & 1910 & $1,317,644$ & M & Q351 & 1941 & 456,179 & \\
\hline Q322 & 1939 & 325,018 & & Q324 & 1911 & $1,229,113$ & $\mathbf{M}$ & Q351 & 1942 & 358,400 & \\
\hline Q322 & 1940 & 373,280 & & Q324 & 1912 & $1,412,971$ & M & Q351 & 1943 & 327,000 & \\
\hline Q322 & 1941 & 380,760 & & Q324 & 1913 & $1,623,109$ & $\mathbf{M}$ & Q351 & 1944 & 350,771 & \\
\hline Q322 & 1942 & 331,075 & & Q324 & 1914 & $1,561,031$ & $\mathbf{M}$ & Q351 & 1945 & 167,717 & \\
\hline Q322 & 1943 & 358,115 & & Q324 & 1915 & $1,513,831$ & $\mathbf{M}$ & Q352 & 1906 & 468,291 & $\mathrm{M}, \mathrm{E}$ \\
\hline Q322 & 1944 & 353,885 & & Q324 & 1916 & 896,751 & $\mathrm{X}, \mathrm{M}$ & Q352 & 1907 & 641,220 & $\mathrm{M}, \mathrm{E}$ \\
\hline Q322 & 1974 & 66,900 & & Q324 & 1917 & $1,376,110$ & $\mathbf{M}$ & Q352 & 1908 & 531,545 & $\mathrm{M}, \mathrm{E}$ \\
\hline Q322 & 1975 & 127,000 & & Q324 & 1918 & $1,494,580$ & $\mathbf{M}$ & Q352 & 1909 & 566,044 & $\mathrm{M}, \mathrm{E}$ \\
\hline Q322 & 1976 & 116,500 & & Q324 & 1919 & $1,466,890$ & M & Q352 & 1910 & 639,860 & $\mathrm{M}, \mathrm{E}$ \\
\hline Q322 & 1977 & 109,500 & & Q324 & 1920 & $1,593,010$ & M & Q352 & 1911 & 663,578 & $\mathrm{M}, \mathrm{E}$ \\
\hline Q322 & 1978 & 79,300 & & Q324 & 1921 & $1,488,810$ & $\mathbf{M}$ & Q352 & 1912 & 734,712 & $\mathrm{M}, \mathrm{E}$ \\
\hline Q322 & 1979 & 57,900 & & Q324 & 1922 & $1,468,720$ & $\mathbf{M}$ & Q352 & 1913 & 754,229 & $\mathrm{M}, \mathrm{E}$ \\
\hline Q322 & 1980 & 41,200 & & Q324 & 1923 & $1,388,610$ & $\mathbf{M}$ & Q352 & 1914 & 800,251 & $\mathrm{M}, \mathrm{E}$ \\
\hline Q322 & 1981 & 65,100 & & Q324 & 1924 & $1,408,540$ & $\mathbf{M}$ & Q352 & 1915 & 828,514 & $\mathrm{M}, \mathrm{E}$ \\
\hline Q322 & 1982 & 108,700 & & Q324 & 1925 & $1,229,350$ & $\mathbf{M}$ & $\mathrm{Q} 352$ & 1916 & 954,358 & $\mathrm{M}, \mathrm{E}$ \\
\hline Q322 & 1983 & 107,800 & & Q324 & 1926 & 994,320 & $\mathrm{X}, \mathrm{M}$ & Q352 & 1917 & $1,037,567$ & $\mathrm{M}, \mathrm{E}$ \\
\hline Q322 & 1984 & 215,200 & & Q324 & 1927 & $1,058,420$ & $\mathbf{M}$ & Q352 & 1918 & $1,201,304$ & $\mathrm{M}, \mathrm{E}$ \\
\hline Q323 & 1933 & 151,711 & & Q324 & 1928 & $1,106,920$ & $M$ & Q352 & 1919 & $1,147,768$ & $\mathrm{M}, \mathrm{E}$ \\
\hline Q323 & 1934 & 352,373 & & Q324 & 1929 & $1,053,210$ & M & Q352 & 1920 & $1,220,453$ & $\mathrm{M}, \mathrm{E}$ \\
\hline Q323 & 1935 & 342,352 & & Q324 & 1930 & 956,610 & $\mathrm{M}$ & Q352 & 1921 & $1,349,199$ & $\mathrm{M}, \mathrm{E}$ \\
\hline Q323 & 1936 & 326,189 & & Q324 & 1931 & 935,630 & $\mathbf{M}$ & Q352 & 1922 & $1,557,915$ & $\mathrm{M}, \mathrm{E}$ \\
\hline Q323 & 1937 & 418,436 & & Q324 & 1932 & 996,230 & $M$ & Q352 & 1923 & $2,001,821$ & $\mathrm{M}, \mathrm{E}$ \\
\hline Q323 & 1938 & 478,953 & & Q324 & 1933 & $1,017,920$ & $\mathbf{M}$ & Q352 & 1924 & $2,272,238$ & $\mathrm{M}, \mathrm{E}$ \\
\hline Q323 & 1939 & 384,806 & . & Q324 & 1934 & 480,130 & M & Q352 & 1925 & $2,372,336$ & $\mathrm{M}, \mathrm{E}$ \\
\hline Q323 & 1940 & 467,775 & & Q324.2 & 1934 & 171,332 & $\mathrm{E}$ & Q352 & 1926 & $2,496,855$ & M \\
\hline Q323 & 1941 & 565,648 & & Q324.2 & 1935 & 326,098 & $\mathrm{E}$ & Q352 & 1927 & $2,532,011$ & M \\
\hline Q323 & 1942 & 561,337 & & Q324.2 & 1936 & 285,677 & $E$ & Q352 & 1928 & $2,651,907$ & M \\
\hline Q323 & 1943 & 575,400 & & Q324.2 & 1937 & 280,077 & $\mathrm{E}$ & Q352 & 1929 & $2,555,041$ & $\mathbf{M}$ \\
\hline
\end{tabular}


Table 2B. Public-supply pumpage in Kings, Queens, and Nassau Counties, N.Y., 1898-1984--continued

\begin{tabular}{|c|c|c|c|c|c|c|c|c|c|c|c|}
\hline $\begin{array}{c}\text { Well } \\
\text { number }\end{array}$ & Year & Pumpage & Remarks & $\begin{array}{c}\text { Well } \\
\text { number }\end{array}$ & Year & Pumpage & Remarks & $\begin{array}{c}\text { Well } \\
\text { number }\end{array}$ & Year & Pumpage & Remarks \\
\hline Q352 & 1930 & $2,743,110$ & $M$ & Q353 & 1930 & 31,963 & $\mathbf{M}$ & Q354 & 1932 & 352,524 & \\
\hline Q352 & 1931 & $2,639,425$ & $\mathbf{M}$ & Q353 & 1931 & 27,080 & $\mathbf{M}$ & Q354 & 1933 & 424,095 & \\
\hline Q352 & 1932 & $2,594,031$ & M & Q353 & 1932 & 17,551 & $\mathbf{M}$ & Q354 & 1934 & 449,426 & \\
\hline Q352 & 1933 & $2,242,151$ & $\mathbf{M}$ & Q353 & 1933 & 41,417 & $\mathbf{M}$ & Q354 & 1935 & 705,132 & \\
\hline Q352 & 1934 & $1,983,722$ & M & Q353 & 1934 & 76,731 & $\mathbf{M}$ & Q354 & 1936 & 784,860 & \\
\hline Q352 & 1935 & $1,210,174$ & $\mathbf{M}$ & Q353 & 1935 & 326,449 & $\mathbf{M}$ & Q354 & 1937 & 775,216 & . \\
\hline Q352 & 1936 & 754,336 & M & Q353 & 1936 & 475,629 & $\mathbf{M}$ & Q354 & 1938 & 778,466 & \\
\hline Q352 & 1937 & 663,986 & $\mathbf{M}$ & Q353 & 1937 & 446,247 & $\mathbf{M}$ & Q354 & 1939 & 820,047 & \\
\hline Q352 & 1938 & 715,587 & $\mathrm{M}$ & Q353 & 1938 & 484,985 & $\mathbf{M}$ & Q354 & 1940 & 804,872 & \\
\hline Q352 & 1939 & 687,623 & M & Q353 & 1939 & 450,559 & $\mathbf{M}$ & Q354 & 1941 & 797,396 & \\
\hline Q352 & 1940 & 488,999 & M & Q353 & 1940 & 372,897 & $\mathbf{M}$ & Q354 & 1942 & 786,900 & \\
\hline Q352 & 1941 & 654,610 & M & Q353 & 1941 & 378,803 & $\mathbf{M}$ & Q354 & 1943 & 788,000 & \\
\hline Q352 & 1942 & 494,300 & M & Q353 & 1942 & 493,200 & $\mathbf{M}$ & Q354 & 1944 & 645,980 & \\
\hline Q352 & 1943 & 568,000 & $\mathbf{M}$ & Q353 & 1943 & 614,000 & $\mathbf{M}$ & Q354 & 1945 & 707,827 & \\
\hline Q352 & 1944 & 544,121 & M & Q353 & 1944 & 679,465 & M & Q354 & 1946 & 698,233 & \\
\hline Q352 & 1945 & 377,303 & $\mathbf{M}$ & Q353 & 1945 & 598,811 & $\mathbf{M}$ & Q354 & 1947 & 699,017 & \\
\hline Q352 & 1946 & 301,222 & $\mathbf{M}$ & Q353 & 1946 & 241,821 & $\mathbf{M}$ & Q354 & 1948 & 703,609 & \\
\hline Q352 & 1947 & 263,864 & $\mathbf{M}$ & Q353 & 1947 & 87,490 & M & Q354 & 1949 & 696,435 & \\
\hline Q352 & 1948 & 412,063 & M & Q353 & 1948 & 106,819 & $\mathbf{M}$ & Q354 & 1950 & 692,405 & \\
\hline Q353 & 1904 & 439,200 & $\mathrm{M}, \mathrm{E}$ & Q353 & 1949 & 281,908 & M & Q354 & 1951 & 692,040 & \\
\hline Q353 & 1905 & 438,000 & $\mathrm{M}, \mathrm{E}$ & Q353 & 1952 & 4,296 & $\mathbf{M}$ & Q354 & 1952 & 686,714 & \\
\hline Q353 & 1906 & 6,209 & $\mathrm{M}, \mathrm{E}$ & Q353 & 1956 & 70,308 & M & Q354 & 1953 & 685,105 & \\
\hline Q353 & 1907 & 8,502 & $\mathrm{M}, \mathrm{E}$ & Q353 & 1957 & 75,470 & $\mathbf{M}$ & Q354 & 1954 & 671,235 & \\
\hline Q353 & 1908 & 7,048 & $\mathrm{M}, \mathrm{E}$ & Q353 & 1958 & 74,424 & $M$ & Q354 & 1955 & 583,530 & \\
\hline Q353 & 1909 & 7,505 & $\mathrm{M}, \mathrm{E}$ & Q353 & 1959 & 54,150 & $\mathbf{M}$ & Q354 & 1956 & 670,870 & \\
\hline Q353 & 1910 & 8,484 & $\mathrm{M}, \mathrm{E}$ & Q353 & 1960 & 107,712 & $\mathbf{M}$ & Q354 & 1957 & 669,615 & \\
\hline Q353 & 1911 & 8,799 & $\mathrm{M}, \mathrm{E}$ & Q353 & 1961 & 162,410 & $\mathbf{M}$ & Q354 & 1958 & 667,950 & \\
\hline Q353 & 1912 & 9,742 & $\mathrm{M}, \mathrm{E}$ & Q353 & 1962 & 24,472 & M & Q354 & 1959 & 659,190 & \\
\hline Q353 & 1913 & 9,870 & $\mathrm{M}, \mathrm{E}$ & Q353 & 1963 & 32,604 & M & Q354 & 1960 & 597,456 & \\
\hline Q353 & 1914 & 10,611 & $\mathrm{M}, \mathrm{E}$ & Q353 & 1964 & 41,710 & $\mathbf{M}$ & Q354 & 1961 & 738,947 & \\
\hline Q353 & 1915 & 10,986 & $\mathrm{M}, \mathrm{E}$ & Q353 & 1965 & 68,968 & $\mathbf{M}$ & Q354 & 1962 & 785,115 & \\
\hline Q353 & 1916 & 12,654 & $\mathrm{M}, \mathrm{E}$ & Q353 & 1966 & 95,944 & $\mathbf{M}$ & Q354 & 1963 & 776,355 & \\
\hline Q353 & 1917 & 13,757 & $\mathrm{M}, \mathrm{E}$ & Q353 & 1967 & 69,973 & $\mathbf{M}$ & Q354 & 1964 & 773,800 & \\
\hline Q353 & 1918 & 15,929 & $\mathrm{M}, \mathrm{E}$ & Q353 & 1968 & 124,292 & $\mathbf{M}$ & Q354 & 1965 & 761,390 & \\
\hline Q353 & 1919 & 15,219 & $\mathrm{M}, \mathrm{E}$ & Q353 & 1969 & 116,234 & $\mathbf{M}$ & Q354 & 1966 & 751,170 & \\
\hline Q353 & 1920 & 16,183 & M,E & Q353 & 1970 & 116,234 & $\mathbf{M}$ & Q354 & 1967 & 747,155 & \\
\hline Q353. & 1921 & 17,890 & M,E & Q353 & 1971 & 101,088 & $\mathbf{M}$ & Q354 & 1968 & 750,075 & \\
\hline Q353 & 1922 & 20,657 & $\mathrm{M}, \mathrm{E}$ & Q353 & 1972 & 4,631 & $\mathbf{M}$ & Q354 & 1969 & 760,624 & \\
\hline Q353 & 1923 & 26,543 & M,E & Q354 & 1925 & 62,868 & $\mathbf{E}$ & Q354 & 1970 & 760,624 & \\
\hline Q353 & 1924 & 30,129 & $\mathrm{M}, \mathrm{E}$ & Q354 & 1926 & 66,165 & & Q354 & 1971 & 807,015 & \\
\hline Q353 & 1925 & 29,436 & $\mathrm{M}, \mathrm{E}$ & Q354 & 1927 & 59,800 & & Q354 & 1972 & 697,452 & \\
\hline Q353 & 1926 & 30,977 & $\mathbf{M}$ & Q354 & 1928 & 112,775 & & Q354 & 1973 & 455,535 & \\
\hline Q353 & 1927 & 29,795 & M & Q354 & 1929 & 218,960 & & Q354 & 1974 & 55,443 & $\mathrm{E}$ \\
\hline Q353 & 1928 & 33,116 & $\mathbf{M}$ & Q354 & 1930 & 295,286 & & Q355 & 1926 & 40,264 & \\
\hline Q353 & 1929 & 31,559 & $\mathbf{M}$ & Q354 & 1931 & 238,901 & & Q355 & 1927 & 65,255 & \\
\hline
\end{tabular}


Table 2B. Public-supply pumpage in Kings, Queens, and Nassau Counties, N.Y., 1898-1984--continued

\begin{tabular}{|c|c|c|c|c|c|c|c|c|c|c|c|}
\hline $\begin{array}{c}\text { Well } \\
\text { number }\end{array}$ & Year & Pumpage & Remarks & $\begin{array}{c}\text { Well } \\
\text { number }\end{array}$ & Year & Pumpage & Remarks & $\begin{array}{c}\text { Well } \\
\text { number }\end{array}$ & Year & Pumpage & Remarks \\
\hline Q355 & 1928 & 95,841 & & Q557 & 1926 & 6,393 & & Q559 & 1928 & 256,180 & \\
\hline Q355 & 1929 & 154,853 & & Q557 & 1929 & 70,750 & & Q559 & 1929 & 282,015 & \\
\hline Q355 & 1930 & 324,534 & & Q557 & 1930 & 34,685 & & Q559 & 1930 & 224,459 & \\
\hline Q355 & 1931 & 132,587 & & Q557 & 1931 & 44,209 & & Q559 & 1931 & 228,017 & \\
\hline Q355 & 1932 & 136,097 & & Q557 & 1932 & 43,980 & & Q559 & 1932 & 227,690 & \\
\hline Q355 & 1933 & 228,068 & & Q557 & 1933 & 73,155 & & Q559 & 1933 & 197,160 & \\
\hline Q355 & 1934 & 407,821 & & Q557 & 1934 & 79,556 & & Q559 & 1934 & 179,916 & \\
\hline Q355 & 1935 & 617,136 & & Q557 & 1935 & 53,831 & & Q559 & 1935 & 225,336 & \\
\hline Q355 & 1936 & 702,761 & & Q557 & 1936 & 30,071 & & Q559 & 1936 & 270,940 & \\
\hline Q355 & 1937 & 628,793 & & Q557 & 1937 & 4,600 & & Q559 & 1937 & 231,692 & \\
\hline Q355 & 1938 & 781,606 & & Q557 & 1938 & 15 & & Q559 & 1938 & 118,788 & \\
\hline Q355 & 1939 & 849,970 & & Q557 & 1939 & 44,784 & & Q559 & 1939 & 137,524 & \\
\hline Q355 & 1940 & 833,831 & & Q557 & 1940 & 8,345 & & Q559 & 1940 & 213,529 & \\
\hline Q355 & 1941 & 803,078 & & Q557 & 1941 & 29,095 & & Q559 & 1941 & 181,840 & \\
\hline Q355 & 1942 & 785,100 & & Q557 & 1942 & 1,647 & & Q559 & 1942 & 330,295 & \\
\hline Q355 & 1943 & 793,000 & & Q557 & 1943 & 33,028 & & Q559 & 1943 & 376,375 & \\
\hline Q355 & 1944 & 761,951 & & Q557 & 1944 & 58,188 & & Q559 & 1944 & 252,580 & \\
\hline Q355 & 1945 & 760,471 & & Q558 & 1930 & 189,397 & & Q560 & 1935 & 94,659 & $\mathrm{E}$ \\
\hline Q355 & 1946 & 753,632 & & Q558 & 1931 & 359,123 & & Q560 & 1936 & 272,220 & $\mathbf{E}$ \\
\hline Q355 & 1947 & 753,642 & & Q558 & 1932 & 479,790 & & Q560 & 1937 & 262,617 & $\mathbf{E}$ \\
\hline Q355 & 1948 & 756,955 & & Q558 & 1933 & 430,998 & & Q560 & 1938 & 261,835 & $\mathbf{E}$ \\
\hline Q355 & 1949 & 759,975 & & Q558 & 1934 & 414,825 & & Q560 & 1939 & 281,257 & $\mathrm{E}$ \\
\hline Q355 & 1950 & 771,610 & & Q558 & 1935 & 408,908 & & Q560 & 1940 & 283,912 & E \\
\hline Q355 & 1951 & 762,120 & & Q558 & 1936 & 285,539 & & Q560 & 1941 & 285,985 & E \\
\hline Q355 & 1952 & 767,595 & & Q558 & 1937 & 351,848 & & Q560 & 1942 & 272,025 & $\mathrm{E}$ \\
\hline Q355 & 1953 & 761,025 & & Q558 & 1938 & 299,884 & & Q560 & 1943 & 293,975 & $\mathbf{E}$ \\
\hline Q355 & 1954 & 751,170 & & Q558 & 1939 & 411,476 & & Q560 & 1944 & 289,530 & E \\
\hline Q355 & 1955 & 742,775 & & Q558 & 1940 & 356,267 & & Q560 & 1974 & 107,833 & $\mathrm{E}$ \\
\hline Q355 & 1956 & 737,300 & & Q558 & 1941 & 335,063 & & Q560 & 1975 & 159,933 & $\mathrm{E}$ \\
\hline Q355 & 1957 & 723,389 & & Q558 & 1942 & 314,115 & & Q560 & 1976 & 89,900 & $\mathrm{E}$ \\
\hline Q355 & 1958 & 714,670 & & Q558 & 1943 & 434,805 & & Q560 & 1977 & 38,000 & E \\
\hline Q355 & 1959 & 716,860 & & Q558 & 1944 & 349,390 & & Q560 & 1978 & 121,500 & E \\
\hline Q355 & 1960 & 725,255 & & Q558 & 1974 & 176,500 & & Q560 & 1979 & 160,333 & E \\
\hline Q355 & 1961 & 641,337 & & Q558 & 1975 & 220,900 & & Q560 & 1980 & 97,267 & $\mathrm{E}$ \\
\hline Q355 & 1962 & 748,250 & & Q558 & 1976 & 219,300 & & Q560 & 1981 & 49,167 & $\mathbf{E}$ \\
\hline Q355 & 1963 & 710,655 & & Q558 & 1977 & 186,400 & & Q560 & 1982 & 107,733 & $\mathrm{E}$ \\
\hline Q355 & 1964 & 661,015 & & Q558 & 1978 & 219,600 & & Q561 & 1935 & 94,659 & $\mathrm{E}$ \\
\hline Q355 & 1965 & 495,830 & & Q558 & 1979 & 191,500 & & Q561 & 1936 & 272,220 & $\mathrm{E}$ \\
\hline Q355 & 1966 & 620,046 & & Q558 & 1980 & 167,800 & & Q561 & 1937 & 262,617 & $\mathrm{E}$ \\
\hline Q355 & 1967 & 560,280 & & Q558 & 1981 & 218,900 & & Q561 & 1938 & 261,835 & $\mathrm{E}$ \\
\hline Q355 & 1968 & 471,215 & & Q558 & 1982 & 213,400 & & Q561 & 1939 & 281,257 & $\mathbf{E}$ \\
\hline Q355 & 1969 & 470,120 & & Q558 & 1983 & 104,500 & & Q561 & 1940 & 283,912 & $\mathbf{E}$ \\
\hline Q355 & 1970 & 470,120 & & Q558 & 1984 & 109,500 & & Q561 & 1941 & 285,985 & E \\
\hline Q355 & 1971 & 445,300 & & Q559 & 1926 & 112,197 & & Q561 & 1942 & 272,025 & $\mathbf{E}$ \\
\hline Q355 & 1972 & 184,191 & & Q559 & 1927 & 122,657 & & Q561 & 1943 & 293,975 & $\mathrm{E}$ \\
\hline
\end{tabular}


Table 2B. Public-supply pumpage in Kings, Queens, and Nassau Counties, N.Y., 1898-1984--continued

\begin{tabular}{|c|c|c|c|c|c|c|c|c|c|c|c|}
\hline $\begin{array}{c}\text { Well } \\
\text { number }\end{array}$ & Year & Pumpage & Remarks & $\begin{array}{c}\text { Well } \\
\text { number }\end{array}$ & Year & Pumpage & Remarks & $\begin{array}{c}\text { Well } \\
\text { number }\end{array}$ & Year & Pumpage & Remarks \\
\hline Q561 & 1944 & 289,530 & $\bar{E}$ & Q563 & 1974 & 27,410 & & Q565 & 1940 & 556,450 & \\
\hline Q561 & 1974 & 107,833 & $\mathrm{E}$ & Q563 & 1975 & 13,450 & & Q565 & 1941 & 548,890 & \\
\hline Q561 & 1975 & 159,933 & $\mathrm{E}$ & Q563 & 1976 & 18,040 & & Q565 & 1942 & 560,940 & \\
\hline Q561 & 1976 & 89,900 & $\mathrm{E}$ & Q563 & 1977 & 25,640 & & Q565 & 1943 & 494,690 & \\
\hline Q561 & 1977 & 38,000 & $\mathrm{E}$ & Q563 & 1978 & 41,520 & & Q565 & 1944 & 442,220 & \\
\hline Q561 & 1978 & 121,500 & $\mathbf{E}$ & Q563 & 1979 & 25,350 & & Q565 & 1974 & 137,500 & \\
\hline Q561 & 1979 & 160,333 & E & Q563 & 1980 & 25,900 & & Q566 & 1929 & 43,578 & \\
\hline Q561 & 1980 & 97,267 & E & Q563 & 1981 & 19,900 & & Q566 & 1930 & 40,121 & \\
\hline Q561 & 1981 & 49,167 & $\mathrm{E}$ & Q563 & 1982 & 25,050 & & Q566 & 1931 & 76,081 & \\
\hline Q561 & 1982 & 107,733 & $\mathrm{E}$ & Q564 & 1931 & 210,110 & & Q566 & 1932 & 145,338 & \\
\hline Q562 & 1935 & 283,978 & & Q564 & 1932 & 506,651 & & Q566 & 1933 & 150,778 & \\
\hline Q562 & 1936 & 816,660 & & Q564 & 1933 & 463,262 & & Q566 & 1934 & 52,301 & \\
\hline Q562 & 1937 & 787,850 & & Q564 & 1934 & 459,899 & & Q566 & 1935 & 23,443 & \\
\hline Q562 & 1938 & 785,499 & & Q564 & 1935 & 464,158 & & Q566 & 1936 & 25,592 & \\
\hline Q562 & 1939 & 843,771 & & Q564 & 1936 & 325,099 & & Q566 & 1937 & 33,725 & \\
\hline Q562 & 1940 & 851,735 & & Q564 & 1937 & 254,062 & & Q566 & 1938 & 21,095 & \\
\hline Q562 & 1941 & 857,955 & & Q564 & 1938 & 343,362 & & Q566 & 1939 & 64,849 & \\
\hline Q562 & 1942 & 816,075 & & Q564 & 1939 & 356,761 & & Q566 & 1940 & 3,760 & \\
\hline Q562 & 1943 & 881,925 & & Q564 & 1940 & 152,593 & & Q566 & 1941 & 24,857 & \\
\hline Q562 & 1944 & 868,590 & & Q564 & 1941 & 214,038 & & Q566 & 1942 & 2,591 & \\
\hline Q562 & 1974 & 974,300 & & Q564 & 1942 & 113,999 & & Q566 & 1943 & 44,754 & \\
\hline Q562 & 1975 & 985,000 & & Q564 & 1943 & 234,918 & & Q566 & 1944 & 152,297 & \\
\hline Q562 & 1976 & 987,300 & & Q564 & 1944 & 338,990 & & Q566 & 1974 & 364,700 & \\
\hline Q562 & 1977 & 962,900 & & Q564 & 1974 & 437,000 & & Q566 & 1975 & 391,100 & \\
\hline Q562 & 1978 & 831,000 & & Q564 & 1975 & 429,900 & & Q566 & 1976 & 427,900 & \\
\hline Q562 & 1979 & 905,300 & & Q564 & 1976 & 433,600 & & Q566 & 1977 & 399,500 & \\
\hline Q562 & 1980 & $1,011,100$ & & Q564 & 1977 & 436,500 & & Q566 & 1978 & 347,400 & \\
\hline Q562 & 1981 & $1,021,000$ & & Q564 & 1978 & 424,500 & & Q566 & 1979 & 343,000 & \\
\hline Q562 & 1982 & $1,071,400$ & & Q564 & 1979 & 376,500 & & Q566 & 1980 & 365,700 & \\
\hline Q562 & 1983 & 911,900 & & Q564 & 1980 & 304,200 & & Q566 & 1981 & 353,200 & \\
\hline Q562 & 1984 & 894,300 & & Q564 & 1981 & 435,500 & & Q566 & 1982 & 148,000 & \\
\hline Q563 & 1931 & 54,858 & & Q564 & 1982 & 326,000 & & Q566 & 1983 & 218,700 & \\
\hline Q563 & 1932 & 95,528 & & Q564 & 1983 & 393,800 & & Q566 & 1984 & 216,900 & \\
\hline Q563 & 1933 & 28,232 & . & Q565 & 1928 & 132,512 & & Q567 & 1930 & 47,038 & \\
\hline Q563 & 1934 & 65,228 & & Q565 & 1929 & 230,709 & & Q567 & 1933 & 6,964 & \\
\hline Q563 & 1935 & 9,008 & & Q565 & 1930 & 302,308 & & Q567 & 1937 & 268,539 & \\
\hline Q563 & 1936 & 10,885 & & Q565 & 1931 & 354,304 & & Q567 & 1938 & 483,365 & \\
\hline Q563 & 1937 & 10,078 & & Q565 & 1932 & 205,590 & & Q567 & 1939 & 488,910 & \\
\hline Q563 & 1938 & 7,961 & & Q565 & 1933 & 124,310 & & Q567 & 1940 & 420,770 & \\
\hline Q563 & 1939 & 36,371 & & Q565 & 1934 & 219,620 & & Q567 & 1941 & 436,040 & \\
\hline Q563 & 1940 & 15,077 & & Q565 & 1935 & 125,778 & & Q567 & 1942 & 404,665 & \\
\hline Q563 & 1941 & 75,495 & & Q565 & 1936 & 84,067 & & Q567 & 1943 & 433,575 & \\
\hline Q563 & 1942 & 89,461 & & Q565 & 1937 & 156,809 & & Q567 & 1944 & 439,685 & \\
\hline Q563 & 1943 & 16,431 & & Q565 & 1938 & 382,582 & & Q567 & 1974 & 361,200 & \\
\hline Q563 & 1944 & 26,596 & & Q565 & 1939 & 540,392 & & Q567 & 1975 & 194,800 & \\
\hline
\end{tabular}


Table 2B. Public-supply pumpage in Kings, Queens, and Nassau Counties, N.Y., 1898-1984--continued

\begin{tabular}{|c|c|c|c|c|c|c|c|c|c|c|c|}
\hline $\begin{array}{c}\text { Well } \\
\text { number }\end{array}$ & Year & Pumpage & Remarks & $\begin{array}{c}\text { Well } \\
\text { number }\end{array}$ & Year & Pumpage & Remarks & $\begin{array}{c}\text { Well } \\
\text { number }\end{array}$ & Year & Pumpage & Remarks \\
\hline Q567 & 1976 & 372,900 & & Q570 & 1940 & 296,195 & $\mathrm{E}$ & Q1378 & 1962 & 151,510 & \\
\hline Q567 & 1977 & 218,600 & & Q570 & 1941 & 357,145 & $\mathrm{E}$ & Q1378 & 1963 & 154,530 & \\
\hline Q567 & 1978 & 167,900 & & Q570 & 1942 & 335,365 & $\mathrm{E}$ & Q1378 & 1964 & 168,399 & \\
\hline Q567 & 1979 & 263,100 & & Q570 & 1943 & 312,448 & $\mathrm{E}$ & Q1378 & 1965 & 60,894 & \\
\hline Q567 & 1980 & 475,100 & & Q570 & 1944 & 333,813 & $\mathrm{E}$ & Q1378 & 1966 & 110,152 & \\
\hline Q567 & 1981 & 277,100 & & Q570 & 1974 & 36,560 & $\mathrm{E}$ & Q1378 & 1967 & 135,534 & \\
\hline Q567 & 1982 & 466,500 & & Q570 & 1975 & 38,000 & $\mathrm{E}$ & Q1378 & 1968 & 88,693 & \\
\hline Q567 & 1983 & 273,100 & & Q570 & 1976 & 72,633 & $\mathrm{E}$ & Q1378 & 1969 & 119,603 & \\
\hline Q567 & 1984 & 242,200 & & Q570 & 1977 & 41,527 & $\mathrm{E}$ & Q1378 & 1970 & 119,603 & \\
\hline Q568 & 1933 & 162,674 & & Q570 & 1978 & 42,100 & $\mathrm{E}$ & Q1378 & 1971 & 294,965 & \\
\hline Q568 & 1934 & 386,391 & & Q570 & 1979 & 26,990 & $\mathrm{E}$ & Q1378 & 1972 & 240,778 & \\
\hline Q568 & 1935 & 339,230 & & Q570 & 1980 & 46,137 & $\mathrm{E}$ & Q1378 & 1973 & 99,953 & \\
\hline Q568 & 1936 & 330,336 & & Q570 & 1981 & 38,713 & $\mathrm{E}$ & Q1378 & 1974 & 55,442 & $\mathrm{E}$ \\
\hline Q568 & 1937 & 411,159 & & Q570 & 1982 & 99,740 & $\mathrm{E}$ & Q1379 & 1946 & 364,983 & \\
\hline Q568 & 1938 & 548,049 & & Q1058 & 1940 & 296,195 & $\mathrm{E}$ & Q1379 & 1947 & 643,611 & \\
\hline Q568 & 1939 & 436,370 & & Q1058 & 1941 & 357,145 & $\mathrm{E}$ & Q1379 & 1948 & 649,805 & \\
\hline Q568 & 1940 & 577,303 & & Q1058 & 1942 & 335,365 & $\mathrm{E}$ & Q1379 & 1949 & 651,367 & \\
\hline Q568 & 1941 & 628,620 & & Q1058 & 1943 & 312,448 & $\mathrm{E}$ & Q1379 & 1950 & 639,480 & \\
\hline Q568 & 1942 & 610,350 & & Q1058 & 1944 & 333,813 & $\mathrm{E}$ & Q1379 & 1951 & 430,440 & \\
\hline Q568 & 1943 & 611,465 & & Q1058 & 1974 & 36,560 & $\mathrm{E}$ & Q1379 & 1952 & 636,925 & \\
\hline Q568 & 1944 & 621,135 & & Q1058 & 1975 & 38,000 & $\mathrm{E}$ & Q1379 & 1953 & 484,554 & \\
\hline Q568 & 1974 & 453,200 & & Q1058 & 1976 & 72,633 & $\mathrm{E}$ & Q1379 & 1954 & 441,657 & \\
\hline Q568 & 1975 & 386,900 & & Q1058 & 1977 & 41,527 & $\mathrm{E}$ & Q1379 & 1955 & 542,775 & \\
\hline Q568 & 1976 & 436,400 & & Q1058 & 1978 & 42,100 & $\mathrm{E}$ & Q1379 & 1956 & 532,170 & \\
\hline Q568 & 1977 & 541,000 & & Q1058 & 1979 & 26,990 & $\mathrm{E}$ & Q1379 & 1957 & 543,136 & \\
\hline Q568 & 1978 & 569,500 & & Q1058 & 1980 & 46,137 & $\mathrm{E}$ & Q1379 & 1958 & 545,310 & \\
\hline Q568 & 1979 & 501,000 & & Q1058 & 1981 & 38,713 & $\mathrm{E}$ & Q1379 & 1959 & 605,535 & \\
\hline Q568 & 1980 & 409,100 & & Q1058 & 1982 & 99,740 & $\mathrm{E}$ & Q1379 & 1960 & 605,170 & \\
\hline Q568 & 1981 & 300,700 & & Q1378 & 1945 & 263,926 & & Q1379 & 1961 & 653,350 & \\
\hline Q568 & 1982 & 707,300 & & Q1378 & 1946 & 684,751 & & Q1379 & 1962 & 727,080 & \\
\hline Q568 & 1983 & 666,600 & & Q1378 & 1947 & 674,056 & & Q1379 & 1963 & 707,735 & \\
\hline Q568 & 1984 & 467,800 & & Q1378 & 1948 & 542,658 & & Q1379 & 1964 & 702,625 & \\
\hline Q569 & 1976 & 72,633 & $\mathrm{E}$ & Q1378 & 1949 & 422,954 & & Q1379 & 1965 & 720,510 & \\
\hline Q569 & 1977 & 41,527 & $\mathrm{E}$ & Q1378 & 1950 & 329,456 & & Q1379 & 1966 & 723,430 & \\
\hline Q569 & 1978 & 42,100 & $\mathrm{E}$ & Q1378 & 1951 & 481,628 & & Q1379 & 1967 & 671,040 & \\
\hline Q569 & 1979 & 26,990 & $\mathrm{E}$ & Q1378 & 1952 & 514,080 & & Q1379 & 1968 & 720,145 & \\
\hline Q569 & 1980 & 46,137 & $\mathrm{E}$ & Q1378 & 1953 & 531,805 & & Q1379 & 1969 & 721,240 & \\
\hline Q569 & 1981 & 38,713 & $\mathrm{E}$ & Q1378 & 1954 & 529,615 & & Q1379 & 1970 & 721,240 & \\
\hline Q569 & 1982 & 99,740 & $\mathrm{E}$ & Q1378 & 1955 & 537,280 & & Q1379 & 1971 & 707,370 & \\
\hline Q570 & 1934 & 171,332 & $\mathrm{E}$ & Q1378 & 1956 & 520,125 & & Q1379 & 1972 & 526,198 & \\
\hline Q570 & 1935 & 326,098 & $\mathrm{E}$ & Q1378 & 1957 & 473,714 & & Q1379 & 1973 & 498,602 & \\
\hline Q570 & 1936 & 285,677 & $\mathrm{E}$ & Q1378 & 1958 & 380,330 & & Q1379 & 1974 & 55,443 & $\mathrm{E}$ \\
\hline Q570 & 1937 & 280,077 & $\mathrm{E}$ & Q1378 & 1959 & 227,997 & & Q1450 & 1974 & 28,340 & \\
\hline Q570 & 1938 & 343,135 & $\mathrm{E}$ & Q1378 & 1960 & 94,464 & & Q1450 & 1975 & 24,800 & \\
\hline Q570 & 1939 & 329,292 & $\mathrm{E}$ & Q1378 & 1961 & 124,292 & & Q1450 & 1976 & 46,500 & \\
\hline
\end{tabular}


Table 2B. Public-supply pumpage in Kings, Queens, and Nassau Counties, N.Y., 1898-1984--continued

\begin{tabular}{|c|c|c|c|c|c|c|c|c|c|c|c|}
\hline $\begin{array}{c}\text { Well } \\
\text { number }\end{array}$ & Year & Pumpage & Remarks & $\begin{array}{c}\text { Well } \\
\text { number }\end{array}$ & Year & Pumpage & Remarks & $\begin{array}{c}\text { Well } \\
\text { number }\end{array}$ & Year & Pumpage & Remarks \\
\hline Q1450 & 1977 & 31,500 & & Q1484 & 1968 & 170,820 & & Q1511 & 1962 & 271,195 & \\
\hline Q1450 & 1978 & 49,600 & & Q1484 & 1969 & 170,090 & & Q1511 & 1963 & 270,465 & \\
\hline Q1450 & 1979 & 24,700 & & Q1484 & 1970 & 170,090 & & Q1511 & 1964 & 467,910 & \\
\hline Q1450 & 1980 & 44,000 & & Q1484 & 1971 & 147,095 & & Q1511 & 1965 & 254,770 & \\
\hline Q1450 & 1981 & 140,500 & & Q1484 & 1972 & 81,184 & & Q1511 & 1966 & 229,950 & \\
\hline Q1450 & 1982 & 138,000 & & Q1484 & 1973 & 11,050 & & Q1511 & 1967 & 305,325 & \\
\hline Q1450 & 1983 & 173,800 & & Q1484 & 1974 & 55,442 & $\mathrm{E}$ & Q1511 & 1968 & 342,735 & \\
\hline Q1450 & 1984 & 182,900 & & Q1493 & 1949 & 59,678 & & Q1511 & 1969 & 334,705 & \\
\hline Q1476 & 1948 & 78,198 & & Q1493 & 1950 & 286 & & Q1511 & 1970 & 334,705 & \\
\hline Q1476 & 1949 & 148,724 & & Q1493 & 1952 & 53,775 & & Q1511 & 1971 & 335,070 & \\
\hline Q1476 & 1950 & 16,008 & & Q1493 & 1953 & 34,830 & & Q1511 & 1972 & 230,378 & \\
\hline Q1476 & 1951 & 31,434 & & Q1493 & 1954 & 65,604 & & Q1511 & 1973 & 275,082 & \\
\hline Q1476 & 1952 & 114,884 & & Q1493 & 1955 & 144,996 & & Q1511 & 1974 & 55,442 & $\mathrm{E}$ \\
\hline Q1476 & 1953 & 59,697 & & Q1493 & 1956 & 262,392 & & Q1528 & 1950 & 19,656 & \\
\hline Q1476 & 1954 & 83,844 & & Q1493 & 1957 & 230,540 & & Q1528 & 1951 & 140,712 & \\
\hline Q1476 & 1955 & 145,672 & & Q1493 & 1958 & 249,106 & & Q1528 & 1952 & 147,492 & \\
\hline Q1476 & 1956 & 107,640 & & Q1493 & 1959 & 203,200 & & Q1528 & 1953 & 363,175 & \\
\hline Q1476 & 1957 & 125,095 & & Q1493 & 1960 & 276,670 & & Q1528 & 1954 & 300,167 & \\
\hline Q1476 & 1958 & 107,559 & & Q1493 & 1961 & 163,836 & & Q1528 & 1955 & 66,599 & \\
\hline Q1476 & 1959 & 2,219 & & Q1493 & 1962 & 162,000 & & Q1528 & 1956 & 47,502 & \\
\hline Q1476 & 1960 & 189,070 & & Q1493 & 1963 & 219,730 & & Q1528 & 1957 & 64,907 & \\
\hline Q1476 & 1961 & 232,596 & & Q1493 & 1964 & 206,955 & & Q1528 & 1958 & 95,232 & \\
\hline Q1476 & 1962 & 246,960 & & Q1493 & 1965 & 228,490 & & Q1528 & 1959 & 332,515 & \\
\hline Q1476 & 1963 & 200,020 & & Q1493 & 1966 & 174,240 & & Q1528 & 1960 & 346,020 & \\
\hline Q1476 & 1964 & 210,970 & & Q1493 & 1967 & 232,166 & & Q1528 & 1961 & 343,830 & \\
\hline Q1476 & 1965 & 188,552 & & Q1493 & 1968 & 302,220 & & Q1528 & 1962 & 342,005 & \\
\hline Q1476 & 1966 & 168,995 & & Q1493 & 1969 & 335,070 & & Q1528 & 1963 & 318,708 & \\
\hline Q1476 & 1967 & 160,114 & & Q1493 & 1970 & 335,070 & & Q1528 & 1964 & 327,066 & \\
\hline Q1476 & 1968 & 193,085 & & Q1493 & 1971 & 262,800 & & Q1528 & 1965 & 293,095 & \\
\hline Q1476 & 1969 & 223,380 & & Q1493 & 1972 & 179,950 & & Q1528 & 1966 & 327,040 & \\
\hline Q1476 & 1970 & 223,380 & & Q1493 & 1973 & 39,618 & & Q1528 & 1967 & 385,123 & \\
\hline Q1476 & 1971 & 249,096 & & Q1493 & 1974 & 55,442 & $\mathrm{E}$ & Q1528 & 1968 & 458,805 & \\
\hline Q1476 & 1972 & 145,580 & & Q1511 & 1949 & 32,133 & & Q1528 & 1969 & 455,885 & \\
\hline Q1476 & 1973 & 20,460 & & Q1511 & 1950 & 290,400 & & Q1528 & 1970 & 455,885 & \\
\hline Q1476 & 1974 & 55,442 & $\mathrm{E}$ & Q1511 & 1951 & 217,617 & & Q1528 & 1971 & 475,230 & \\
\hline Q1477 & 1949 & 97,711 & & Q1511 & 1952 & 111,048 & & Q1528 & 1972 & 421,095 & \\
\hline Q1477 & 1966 & 3,408 & & Q1511 & 1953 & 217,392 & & Q1528 & 1973 & 467,704 & \\
\hline Q1477 & 1967 & 1,032 & & Q1511 & 1954 & 274,456 & & Q1528 & 1974 & 55,443 & $\mathrm{E}$ \\
\hline Q1477 & 1968 & 1,800 & & Q1511 & 1955 & 274,456 & & Q1534 & 1974 & 29,600 & \\
\hline Q1477 & 1969 & 1,800 & & Q1511 & 1956 & 151,174 & & Q1534 & 1975 & 23,700 & \\
\hline Q1477 & 1970 & 1,800 & & Q1511 & 1957 & 172,145 & & Q1534 & 1979 & 2,900 & \\
\hline Q1477 & 1971 & 11,886 & & Q1511 & 1958 & 200,480 & & Q1600 & 1974 & 322,200 & \\
\hline Q1477 & 1972 & 3,723 & & Q1511 & 1959 & 197,327 & & Q1600 & 1975 & 499,500 & \\
\hline Q1484 & 1949 & 221,226 & & Q1511 & 1960 & 262,800 & & Q1600 & 1976 & 603,300 & \\
\hline Q1484 & 1950 & 76,433 & & Q1511 & 1961 & 299,665 & & Q1600 & 1977 & 601,600 & \\
\hline
\end{tabular}


Table 2B. Public-supply pumpage in Kings, Queens, and Nassau Counties, N.Y., 1898-1984--continued

\begin{tabular}{|c|c|c|c|c|c|c|c|c|c|c|c|}
\hline $\begin{array}{c}\text { Well } \\
\text { number }\end{array}$ & Year & Pumpage & Remarks & $\begin{array}{c}\text { Well } \\
\text { number }\end{array}$ & Year & Pumpage & Remarks & $\begin{array}{c}\text { Well } \\
\text { number }\end{array}$ & Year & Pumpage & Remarks \\
\hline Q1600 & 1978 & 601,400 & & Q1747 & 1978 & 492,200 & & Q1840 & 1981 & 396,500 & \\
\hline Q1600 & 1979 & 601,900 & & Q1747 & 1979 & 483,200 & & Q1840 & 1982 & 473,100 & \\
\hline Q1600 & 1980 & 603,600 & & Q1747 & 1980 & 428,000 & & Q1840 & 1983 & 443,200 & \\
\hline Q1600 & 1981 & 601,700 & & Q1747 & 1981 & 442,100 & & Q1840 & 1984 & 474,900 & \\
\hline Q1600 & 1982 & 600,600 & & Q1747 & 1982 & 502,500 & & Q1843 & 1974 & 103,500 & \\
\hline Q1600 & 1983 & 472,000 & & Q1747 & 1983 & 510,000 & & Q1843 & 1975 & 51,800 & \\
\hline Q1600 & 1984 & 578,400 & & Q1747 & 1984 & 512,200 & & Q1843 & 1976 & 93,000 & \\
\hline Q1604. & 1950 & 17,787 & & Q1811 & 1974 & 437,400 & & Q1843 & 1977 & 100,900 & \\
\hline Q1604 & 1951 & 140,336 & & Q1811 & 1975 & 435,200 & & Q1843 & 1978 & 99,800 & \\
\hline Q1604 & 1952 & 82,705 & & Q1811 & 1976 & 475,400 & & Q1843 & 1979 & 35,000 & \\
\hline Q1604 & 1953 & 111,843 & & Q1811 & 1977 & 501,800 & & Q1843 & 1980 & 37,200 & \\
\hline Q1604 & 1954 & 124,503 & & Q1811 & 1978 & 583,400 & & Q1843 & 1981 & 42,700 & \\
\hline Q1604 & 1955 & 191,475 & & Q1811 & 1979 & 577,300 & & Q1843 & 1983 & 500 & \\
\hline Q1604 & 1956 & 89,832 & & Q1811 & 1980 & 406,500 & & Q1957 & 1974 & 816,000 & \\
\hline Q1604 & 1957 & 254,932 & & Q1811 & 1981 & 506,100 & & Q1957 & 1975 & 429,900 & \\
\hline Q1604 & 1958 & 295,285 & & Q1811 & 1982 & 329,400 & & Q1957 & 1976 & 807,600 & \\
\hline Q1604 & 1959 & 255,608 & & Q1811 & 1983 & 124,000 & & Q1957 & 1977 & 781,900 & \\
\hline Q1604 & 1960 & 271,560 & & Q1811 & 1984 & 37,500 & & Q1957 & 1978 & 786,700 & \\
\hline Q1604 & 1961 & 250,390 & & Q1815 & 1974 & 620,200 & & Q1957 & 1979 & 702,300 & \\
\hline Q1604 & 1962 & 240,516 & & Q1815 & 1975 & 609,900 & & Q1957 & 1980 & 786,100 & \\
\hline Q1604 & 1963 & 346,020 & & Q1815 & 1976 & 621,800 & & Q1957 & 1981 & 785,000 & \\
\hline Q1604 & 1964 & 329,960 & & Q1815 & 1977 & 613,000 & & Q1957 & 1982 & 776,500 & \\
\hline Q1604 & 1965 & 311,710 & & Q1815 & 1978 & 521,400 & & Q1957 & 1983 & 693,300 & \\
\hline Q1604 & 1966 & 288,350 & & Q1815 & 1979 & 620,200 & & Q1957 & 1984 & 640,100 & \\
\hline Q1604 & 1967 & 276,900 & & Q1815 & 1980 & 620,600 & & Q1958 & 1974 & 154,300 & \\
\hline Q1604 & 1968 & 311,345 & & Q1815 & 1981 & 495,300 & & Q1958 & 1975 & 348,900 & \\
\hline Q1604 & 1969 & 300,760 & & Q1815 & 1982 & 616,800 & & Q1958 & 1976 & 460,600 & \\
\hline Q1604 & 1970 & 300,760 & & Q1815 & 1983 & 619,100 & & Q1958 & 1977 & 229,800 & \\
\hline Q1604 & 1971 & 278,130 & & Q1815 & 1984 & 618,400 & & Q1958 & 1978 & 241,500 & \\
\hline Q1604 & 1972 & 227,745 & & Q1839 & 1974 & 107,833 & $\mathrm{E}$ & Q1958 & 1979 & 567,900 & \\
\hline Q1604 & 1973 & 217,116 & & Q1839 & 1975 & 159,933 & $\mathrm{E}$ & Q1958 & 1980 & 471,400 & \\
\hline Q1604 & 1974 & 55,443 & $\mathrm{E}$ & Q1839 & 1976 & 89,900 & $\mathrm{E}$ & Q1958 & 1981 & 800,300 & \\
\hline Q1629 & 1974 & 830,400 & & Q1839 & 1977 & 38,000 & $\mathrm{E}$ & Q1958 & 1982 & 793,700 & \\
\hline Q1629 & 1975 & 674,000 & & Q1839 & 1978 & 121,500 & $\mathrm{E}$ & Q1958 & 1983 & 728,600 & \\
\hline Q1629 & 1976 & 743,700 & & Q1839 & 1979 & 160,333 & $\mathrm{E}$ & Q1958 & 1984 & 677,000 & \\
\hline Q1629 & 1977 & 774,600 & & Q1839 & 1980 & 97,267 & $\mathrm{E}$ & Q1997 & 1974 & 408,300 & \\
\hline Q1629 & 1978 & 680,600 & & Q1839 & 1981 & 49,167 & $\mathrm{E}$ & Q1997 & 1975 & 511,500 & \\
\hline Q1629 & 1979 & 591,900 & & Q1839 & 1982 & 107,733 & $\mathrm{E}$ & Q1997 & 1976 & 404,700 & \\
\hline Q1629 & 1980 & 399,300 & & Q1840 & 1974 & 461,100 & & Q1997 & 1977 & 392,200 & \\
\hline Q1629 & 1981 & 98,100 & & Q1840 & 1975 & 497,700 & & Q1997 & 1978 & 474,300 & \\
\hline Q1629 & 1982 & 140,100 & & Q1840 & 1976 & 511,800 & & Q1997 & 1979 & 473,800 & \\
\hline Q1747 & 1974 & 511,000 & & Q1840 & 1977 & 424,300 & & Q1997 & 1980 & 398,400 & \\
\hline Q1747 & 1975 & 502,100 & & Q1840 & 1978 & 473,900 & & Q1997 & 1981 & 474,400 & \\
\hline Q1747 & 1976 & 507,600 & & Q1840 & 1979 & 423,300 & & Q1997 & 1982 & 473,500 & \\
\hline Q1747 & 1977 & 496,400 & & Q1840 & 1980 & 378,800 & & Q1997 & 1983 & 474,000 & \\
\hline
\end{tabular}


Table 2B. Public-supply pumpage in Kings, Queens, and Nassau Counties, N.Y., 1898-1984--continued

\begin{tabular}{|c|c|c|c|c|c|c|c|c|c|c|c|}
\hline $\begin{array}{c}\text { Well } \\
\text { number }\end{array}$ & Year & Pumpage & Remarks & $\begin{array}{c}\text { Well } \\
\text { number }\end{array}$ & Year & Pumpage & Remarks & $\begin{array}{c}\text { Well } \\
\text { number }\end{array}$ & Year & Pumpage & Remarks \\
\hline Q1997 & 1984 & 475,500 & & Q2027 & 1976 & 44,100 & & Q2188 & 1978 & 802,500 & \\
\hline Q2000 & 1974 & 84,200 & & Q2027 & 1977 & 12,240 & & Q2188 & 1979 & 798,600 & \\
\hline Q2000 & 1975 & 88,100 & & Q2027 & 1978 & 10,760 & & Q2188 & 1980 & 804,800 & \\
\hline Q2000 & 1976 & 106,300 & & Q2027 & 1979 & 5,900 & & Q2188 & 1981 & 802,700 & \\
\hline Q2000 & 1977 & 163,700 & & Q2027 & 1980 & 17,800 & & Q2188 & 1982 & 801,900 & \\
\hline Q2000 & 1978 & 221,400 & & Q2027 & 1981 & 39,600 & & Q2188 & 1983 & 620,100 & \\
\hline Q2000 & 1979 & 103,000 & & Q2027 & 1982 & 44,600 & & Q2188 & 1984 & 804,900 & \\
\hline Q2000 & 1980 & 139,500 & & Q2027 & 1983 & 145,200 & & Q2189 & 1974 & 380,200 & \\
\hline Q2000 & 1981 & 255,500 & & Q2027 & 1984 & 146,400 & & Q2189 & 1975 & 334,500 & \\
\hline Q2000 & 1982 & 255,300 & & Q2028 & 1974 & 725,100 & & Q2189 & 1976 & 285,600 & \\
\hline Q2000 & 1983 & 216,700 & & Q2028 & 1975 & 721,500 & & Q2189 & 1977 & 247,900 & \\
\hline Q2000 & 1984 & 231,400 & & Q2028 & 1976 & 802,400 & & Q2189 & 1978 & 256,000 & \\
\hline Q2001 & 1974 & 253,300 & & Q2028 & 1977 & 706,400 & & Q2189 & 1979 & 309,700 & \\
\hline Q2001 & 1975 & 135,800 & & Q2028 & 1978 & 613,300 & & Q2189 & 1980 & 419,400 & \\
\hline Q2001 & 1976 & 117,000 & & Q2028 & 1979 & 744,900 & & Q2189 & 1981 & 510,800 & \\
\hline Q200I & 1977 & 165,000 & & Q2028 & 1980 & 795,600 & & Q2189 & 1982 & 510,800 & \\
\hline Q2001 & 1978 & 211,300 & & Q2028 & 1981 & 714,800 & & Q2189 & 1983 & 510,600 & \\
\hline Q2001 & 1979 & 154,900 & & Q2028 & 1982 & 818,900 & & Q2189 & 1984 & 512,400 & \\
\hline Q2001 & 1980 & 253,000 & & Q2028 & 1983 & 716,800 & & Q2243 & 1974 & 80,500 & \\
\hline Q2001 & 1981 & 181,000 & & Q2028 & 1984 & 650,400 & & Q2243 & 1975 & 59,400 & \\
\hline Q2001 & 1982 & 190,200 & & Q2137 & 1974 & 78,200 & & Q2243 & 1976 & 25,500 & \\
\hline Q2001 & 1983 & 321,100 & & Q2137 & 1975 & 67,400 & & Q2243 & 1977 & 43,870 & \\
\hline Q2001 & 1984 & 238,100 & & Q2137 & 1976 & 50,400 & & Q2243 & 1978 & 35,500 & \\
\hline Q2006 & 1974 & 383,000 & & Q2137 & 1978 & 5,100 & & Q2243 & 1979 & 20,900 & \\
\hline Q2006 & 1975 & 350,800 & & Q2137 & 1979 & 66,800 & & Q2243 & 1980 & 54,840 & \\
\hline Q2006 & 1976 & 329,300 & & Q2137 & 1980 & 136,300 & & Q2243 & 1981 & 42,800 & \\
\hline Q2006 & 1977 & 201,100 & & Q2137 & 1981 & 88,900 & & Q2243 & 1982 & 133,800 & \\
\hline Q2006 & 1978 & 202,000 & & Q2137 & 1982 & 174,900 & & Q2243 & 1983 & 65,550 & \\
\hline Q2006 & 1979 & 193,100 & & Q2137 & 1983 & 105,400 & & Q2243 & 1984 & 11,480 & \\
\hline Q2006 & 1980 & 51,500 & & Q2137 & 1984 & 184,000 & & Q2275 & 1974 & 39,100 & \\
\hline Q2006 & 1981 & 66,000 & & Q2138 & 1974 & 475,800 & & Q2275 & 1975 & 24,100 & \\
\hline Q2006 & 1982 & 85,600 & & Q2138 & 1975 & 482,500 & & Q2275 & 1976 & 62,500 & \\
\hline Q2026 & 1974 & 571,600 & & Q2138 & 1976 & 529,300 & & Q2275 & 1977 & 88,900 & \\
\hline Q2026 & 1975 & 379,100 & & Q2138 & 1977 & 527,700 & & Q2275 & 1978 & 62,000 & \\
\hline Q2026 & 1976 & 287,700 & & Q2138 & 1978 & 424,300 & & Q2275 & 1979 & 24,200 & \\
\hline Q2026 & 1977 & 481,400 & & Q2138 & 1979 & 473,000 & & Q2275 & 1980 & 96,500 & \\
\hline Q2026 & 1978 & 520,400 & & Q2138 & 1980 & 475,200 & & Q2275 & 1981 & 115,200 & \\
\hline Q2026 & 1979 & 437,000 & & Q2138 & 1981 & 473,000 & & Q2275 & 1982 & 148,000 & \\
\hline Q2026 & 1980 & 470,200 & & Q2138 & 1982 & 473,700 & & Q2275 & 1983 & 56,400 & \\
\hline Q2026 & 1981 & 632,700 & & Q2138 & 1983 & 472,700 & & Q2276 & 1974 & 190,500 & \\
\hline Q2026 & 1982 & 671,600 & & Q2138 & 1984 & 416,400 & & Q2276 & 1975 & 162,000 & \\
\hline Q2026 & 1983 & 429,900 & & Q2188 & 1974 & 731,900 & & Q2276 & 1976 & 182,200 & \\
\hline Q2026 & 1984 & 658,400 & & Q2188 & 1975 & 724,800 & & Q2276 & 1977 & 155,300 & \\
\hline Q2027 & 1974 & 45,800 & & Q2188 & 1976 & 645,500 & & Q2276 & 1978 & 157,100 & \\
\hline Q2027 & 1975 & 23,600 & & Q2188 & 1977 & 546,600 & & Q2276 & 1979 & 85,000 & \\
\hline
\end{tabular}


Table 2B. Public-supply pumpage in Kings, Queens, and Nassau Counties, N.Y., 1898-1984--continued

\begin{tabular}{|c|c|c|c|c|c|c|c|c|c|c|c|}
\hline $\begin{array}{c}\text { Well } \\
\text { number }\end{array}$ & Year & Pumpage & Remarks & $\begin{array}{c}\text { Well } \\
\text { number }\end{array}$ & Year & Pumpage & Remarks & $\begin{array}{c}\text { Well } \\
\text { number }\end{array}$ & Year & Pumpage & Remarks \\
\hline Q2276 & 1980 & 184,300 & & Q2343 & 1975 & 738,800 & & Q2374 & 1977 & 498,900 & \\
\hline Q2276 & 1981 & 431,700 & & Q2343 & 1976 & 758,000 & & Q2374 & 1978 & 491,000 & \\
\hline Q2276 & 1982 & 755,200 & & Q2343 & 1977 & 767,000 & & Q2374 & 1979 & 491,300 & \\
\hline Q2276 & 1983 & 631,100 & & Q2343 & 1978 & 681,500 & & Q2374 & 1980 & 494,100 & \\
\hline Q2276 & 1984 & 731,700 & & Q2343 & 1979 & 519,900 & & Q2374 & 1981 & 492,700 & \\
\hline Q2299 & 1974 & 66,400 & & Q2343 & 1980 & 501,000 & & Q2374 & 1982 & 479,800 & \\
\hline Q2299 & 1975 & 54,700 & & Q2343 & 1981 & 235,100 & & Q2374 & 1983 & 421,500 & \\
\hline Q2299 & 1976 & 134,600 & & Q2343 & 1982 & 310,200 & & Q2374 & 1984 & 474,800 & \\
\hline Q2299 & 1977 & 266,900 & & Q2343 & 1983 & 385,600 & & Q2408 & 1974 & 293,700 & \\
\hline Q2299 & 1978 & 405,600 & & Q2362 & 1974 & 518,600 & & Q2408 & 1975 & 193,600 & \\
\hline Q2299 & 1979 & 616,700 & & Q2362 & 1975 & 501,700 & & Q2408 & 1976 & 368,500 & \\
\hline Q2299 & 1980 & 621,900 & & Q2362 & 1976 & 507,500 & & Q2408 & 1977 & 240,900 & \\
\hline Q2299 & 1981 & 353,400 & & Q2362 & 1977 & 451,100 & & Q2408 & 1978 & 333,200 & \\
\hline Q2299 & 1982 & 254,900 & & Q2362 & 1978 & 410,400 & & Q2408 & 1979 & 345,700 & \\
\hline Q2300 & 1974 & 753,200 & & Q2362 & 1979 & 510,700 & & Q2408 & 1980 & 347,100 & \\
\hline Q2300 & 1975 & 600,000 & & Q2362 & 1980 & 511,500 & & Q2408 & 1981 & 346,600 & \\
\hline Q2300 & 1976 & 657,700 & & Q2362 & 1981 & 510,700 & & Q2408 & 1982 & 343,600 & \\
\hline Q2300 & 1977 & 743,200 & & Q2362 & 1982 & 510,500 & & Q2408 & 1983 & 324,400 & \\
\hline Q2300 & 1978 & 778,700 & & Q2362 & 1983 & 511,000 & & Q2408 & 1984 & 59,200 & \\
\hline $\mathrm{Q} 2300$ & 1979 & 801,300 & & Q2362 & 1984 & 443,900 & & Q2409 & 1974 & 360,200 & \\
\hline Q2300 & 1980 & 801,200 & & Q2363 & 1974 & 97,500 & & Q2409 & 1975 & 363,900 & \\
\hline Q2300 & 1981 & 466,800 & & Q2363 & 1975 & 46,600 & & Q2409 & 1976 & 363,800 & \\
\hline Q2300 & 1982 & 198,100 & & Q2363 & 1976 & 140,700 & & Q2409 & 1977 & 306,400 & \\
\hline Q2300 & 1983 & 3,500 & & Q2363 & 1977 & 202,100 & & Q2409 & 1978 & 290,900 & \\
\hline Q2321 & 1974 & 82,700 & & Q2363 & 1978 & 232,800 & & Q2409 & 1979 & 290,800 & \\
\hline Q2321 & 1975 & 48,300 & & Q2363 & 1979 & 151,100 & & Q2409 & 1980 & 292,000 & \\
\hline Q2321 & 1976 & 125,800 & & Q2363 & 1980 & 136,800 & & Q2409 & 1981 & 291,800 & \\
\hline Q2321 & 1977 & 210,400 & & Q2363 & 1981 & 227,800 & & Q2409 & 1982 & 390,200 & \\
\hline Q2321 & 1978 & 266,100 & & Q2363 & 1982 & 255,300 & & Q2409 & 1983 & 497,300 & \\
\hline Q2321 & 1979 & 107,900 & & Q2363 & 1983 & 255,100 & & Q2409 & 1984 & 511,900 & \\
\hline Q2321 & 1980 & 245,000 & & Q2363 & 1984 & 158,300 & & Q2432 & 1974 & 818,100 & \\
\hline Q2321 & 1981 & 502,400 & & Q2373 & 1974 & 288,200 & & Q2432 & 1975 & 852,200 & \\
\hline Q2321 & 1982 & 618,700 & & Q2373 & 1975 & 366,600 & & Q2432 & 1976 & 832,700 & \\
\hline Q2332 & 1974 & 471,200 & & Q2373 & 1976 & 369,100 & & Q2432 & 1977 & 802,900 & \\
\hline Q2332 & 1975 & 585,600 & & Q2373 & 1977 & 302,000 & & Q2432 & 1978 & 800,800 & \\
\hline Q2332 & 1976 & 607,500 & & Q2373 & 1978 & 336,700 & & Q2432 & 1979 & 801,900 & \\
\hline Q2332 & 1977 & 541,700 & & Q2373 & 1979 & 345,700 & & Q2432 & 1980 & 804,000 & \\
\hline Q2332 & 1978 & 474,500 & & Q2373 & 1980 & 346,700 & & Q2432 & 1981 & 801,500 & \\
\hline Q2332 & 1979 & 474,000 & & Q2373 & 1981 & 346,700 & & Q2432 & 1982 & 800,300 & \\
\hline Q2332 & 1980 & 475,600 & & Q2373 & 1982 & 344,800 & & Q2432 & 1983 & 799,100 & \\
\hline Q2332 & 1981 & 473,600 & & Q2373 & 1983 & 304,600 & & Q2432 & 1984 & 800,800 & \\
\hline Q2332 & 1982 & 401,600 & & Q2373 & 1984 & 301,000 & & Q2435 & 1974 & 491,500 & \\
\hline Q2332 & 1983 & 474,200 & & Q2374 & 1974 & 511,800 & & Q2435 & 1975 & 555,200 & \\
\hline Q2332 & 1984 & 474,700 & & Q2374 & 1975 & 494,600 & & Q2435 & 1976 & 467,000 & \\
\hline Q2343 & 1974 & 722,100 & & Q2374 & 1976 & 477,100 & & Q2435 & 1977 & 503,500 & \\
\hline
\end{tabular}


Table 2B. Public-supply pumpage in Kings, Queens, and Nassau Counties, N.Y., 1898-1984--continued

\begin{tabular}{|c|c|c|c|c|c|c|c|c|c|c|c|}
\hline $\begin{array}{c}\text { Well } \\
\text { number }\end{array}$ & Year & Pumpage & Remarks & $\begin{array}{c}\text { Well } \\
\text { number }\end{array}$ & Year & Pumpage & Remarks & $\begin{array}{c}\text { Well } \\
\text { number }\end{array}$ & Year & Pumpage & Remarks \\
\hline Q2435 & 1978 & 443,600 & & Q3014 & 1980 & 437,900 & & N12 & 1980 & 156,700 & \\
\hline Q2435 & 1979 & 484,900 & & Q3014 & 1981 & 437,900 & & $\mathrm{~N} 12$ & 1981 & 224,100 & \\
\hline Q2435 & 1980 & 486,500 & & Q3014 & 1982 & 388,700 & & N12 & 1982 & 196,000 & \\
\hline Q2435 & 1981 & 486,700 & & Q3014 & 1983 & 473,400 & & N12 & 1983 & 288,000 & \\
\hline Q2435 & 1982 & 224,700 & & Q3014 & 1984 & 475,300 & & $\mathrm{~N} 12$ & 1984 & 142,100 & \\
\hline Q2435 & 1983 & 410,700 & 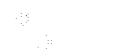 & Q3034 & 1974 & 543,000 & & $\mathrm{~N} 13$ & 1974. & 403,550 & E \\
\hline Q2435 & 1984 & 464,400 & & Q3034 & 1975 & 635,700 & & $\mathrm{~N} 13$ & 1975 & 320,850 & E \\
\hline Q2442 & 1974 & 52,300 & & Q3034 & 1976 & 698,300 & & N13 & 1976 & 257,600 & $\mathrm{E}$ \\
\hline Q2442 & 1975 & 183,700 & & Q3034 & 1977 & 698,300 & & N13 & 1977 & 224,700 & E \\
\hline Q2442 & 1976 & 73,800 & & Q3034 & 1978 & 693,100 & & N13 & 1978 & 373,400 & $\mathrm{E}$ \\
\hline Q2442 & 1977 & 100,000 & & Q3034 & 1979 & 691,300 & & N13 & 1979 & 338,300 & $\mathrm{E}$ \\
\hline Q2442 & 1978 & 84,500 & & Q3034 & 1980 & 687,500 & & N13 & 1980 & 330,000 & $\mathrm{E}$ \\
\hline Q2442 & 1979 & 48,200 & & Q3034 & 1981 & 664,100 & & N13 & 1981 & 245,300 & $\mathrm{E}$ \\
\hline Q2442 & 1980 & 115,300 & & Q3034 & 1982 & 692,600 & & N13 & 1982 & 288,300 & $\mathrm{E}$ \\
\hline Q2442 & 1981 & 68,800 & & Q3034 & 1983 & 693,200 & & N13 & 1983 & 285,850 & $\mathrm{E}$ \\
\hline Q2442 & 1982 & 93,100 & & Q3034 & 1984 & 694,900 & & N13 & 1984 & 240,450 & $\mathrm{E}$ \\
\hline Q2442 & 1983 & 4,100 & & Q3062 & 1975 & 88,380 & & N14 & 1943 & 141,541 & \\
\hline Q2443 & 1974 & 291,500 & & Q3062 & 1976 & 641,100 & & N14 & 1944 & 229,002 & \\
\hline Q2443 & 1975 & 272,700 & & Q3062 & 1977 & 674,600 & & N14 & 1974 & 27,600 & \\
\hline Q2443 & 1976 & 204,700 & & Q3062 & 1978 & 693,200 & & $\mathrm{~N} 14$ & 1975 & 3,100 & \\
\hline Q2443 & 1977 & 303,500 & & Q3062 & 1979 & 692,500 & & N14 & 1976 & 19,300 & \\
\hline Q2443 & 1978 & 260,600 & & Q3062 & 1980 & 661,200 & & $\mathrm{~N} 14$ & 1977 & 18,900 & \\
\hline Q2443 & 1979 & 168,300 & & Q3062 & 1981 & 626,900 & & $\mathrm{~N} 14$ & 1978 & 26,700 & \\
\hline Q2443 & 1980 & 436,000 & & Q3062 & 1982 & 636,100 & & $\mathrm{~N} 14$ & 1979 & 26,300 & \\
\hline Q2443 & 1981 & 279,600 & & Q3062 & 1983 & 690,800 & & $\mathrm{~N} 14$ & 1980 & 39,500 & \\
\hline Q2443 & 1982 & 483,300 & & Q3062 & 1984 & 625,400 & & $\mathrm{~N} 14$ & 1981 & 8,000 & \\
\hline Q2443 & 1983 & 478,800 & & Q3069 & 1977 & 334,600 & & $\mathrm{~N} 14$ & 1982 & 30,100 & \\
\hline Q2443 & 1984 & 284,200 & & Q3069 & 1978 & 510,400 & & N14 & 1983 & 44,500 & \\
\hline Q2955 & 1974 & 298,100 & & Q3069 & 1979 & 491,700 & & $\mathrm{~N} 14$ & 1984 & 3,900 & \\
\hline Q2955 & 1975 & 208,100 & & Q3069 & 1980 & 507,500 & & N15 & 1974 & 13,600 & \\
\hline Q2955 & 1976 & 236,500 & & Q3069 & 1981 & 475,400 & & N15 & 1975 & 900 & \\
\hline Q2955 & 1977 & 249,700 & & Q3069 & 1982 & 509,500 & & N15 & 1976 & 7,200 & \\
\hline Q2955 & 1978 & 250,400 & & Q3069 & 1983 & 509,000 & & N15 & 1977 & 8,000 & \\
\hline Q2955 & 1979 & 248,500 & & Q3069 & 1984 & 392,300 & & N15 & 1978 & 8,000 & \\
\hline Q2955 & 1980 & 352,700 & & Q3083 & 1980 & 303,500 & & N17 & 1974 & 449,600 & \\
\hline Q2955 & 1981 & 361,500 & & Q3083 & 1981 & 273,400 & & N17 & 1975 & 470,200 & \\
\hline Q2955 & 1982 & 550,300 & & Q3083 & 1982 & 94,200 & & $\mathrm{~N} 17$ & 1976 & 473,200 & \\
\hline Q2955 & 1983 & 361,800 & & Q3083 & 1983 & 15,800 & & N17 & 1977 & 472,400 & \\
\hline Q2955 & 1984 & 264,200 & & Q3083 & 1984 & 3,200 & & N17 & 1978 & 262,500 & \\
\hline Q3014 & 1974 & 443,700 & & N12 & 1974 & 167,100 & & N17 & 1979 & 193,800 & \\
\hline Q3014 & 1975 & 433,900 & & $\mathrm{~N} 12$ & 1975 & 235,500 & & N17 & 1980 & 123,700 & \\
\hline Q3014 & 1976 & 441,100 & & N12 & 1976 & 262,100 & & N17 & 1981 & 224,300 & \\
\hline Q3014 & 1977 & 439,000 & & $\mathrm{~N} 12$ & 1977 & 207,600 & & $\mathrm{~N} 17$ & 1982 & 251,000 & \\
\hline Q3014 & 1978 & 437,900 & & $\mathrm{~N} 12$ & 1978 & 258,400 & & N17 & 1983 & 245,500 & \\
\hline Q3014 & 1979 & 417,400 & & $\mathrm{~N} 12$ & 1979 & 264,200 & & N17 & 1984 & 171,100 & \\
\hline
\end{tabular}


Table 2B. Public-supply pumpage in Kings, Queens, and Nassau Counties, N.Y., 1898-1984--continued

\begin{tabular}{|c|c|c|c|c|c|c|c|c|c|c|c|}
\hline $\begin{array}{c}\text { Well } \\
\text { number }\end{array}$ & Year & Pumpage & Remarks & $\begin{array}{c}\text { Well } \\
\text { number }\end{array}$ & Year & Pumpage & Remarks & $\begin{array}{c}\text { Well } \\
\text { number }\end{array}$ & Year & Pumpage & Remarks \\
\hline $\mathrm{N} 22$ & 1980 & 138,660 & & N72 & 1984 & 101,275 & & N97 & 1983 & 92,360 & \\
\hline $\mathrm{N} 22$ & 1981 & 88,252 & & N75 & 1980 & 157,128 & & N97 & 1984 & 144,073 & \\
\hline $\mathrm{N} 22$ & 1982 & 108,195 & & N75 & 1981 & 151,310 & & N101 & 1980 & 127,244 & \\
\hline $\mathrm{N} 22$ & 1983 & 193,296 & & N75 & 1982 & 214,260 & & N101 & 1981 & 121,966 & \\
\hline $\mathrm{N} 22$ & 1984 & 80,867 & & N75 & 1983 & 190,357 & & N101 & 1982 & 108,503 & \\
\hline $\mathrm{N} 24$ & 1980 & 309,790 & & N75 & 1984 & 217,675 & & N101 & 1983 & 60,719 & \\
\hline $\mathrm{N} 24$ & 1981 & 73,630 & & N76 & 1980 & 30,116 & & $\mathrm{~N} 101$ & 1984 & 171,353 & \\
\hline N28 & 1980 & 37,477 & & N76 & 1981 & 13,496 & & N103 & 1980 & 191,320 & \\
\hline N28 & 1981 & 34,272 & & N76 & 1982 & 14,132 & & N103 & 1981 & 184,350 & \\
\hline $\mathrm{N} 28$ & 1982 & 26,357 & & N76 & 1983 & 10,584 & & N103 & 1982 & 225,700 & \\
\hline N28 & 1983 & 29,739 & & N79 & 1980 & 424,193 & & N103 & 1983 & 276,340 & \\
\hline N28 & 1984 & 33,772 & & N79 & 1981 & 163,125 & & N103 & 1984 & 298,740 & \\
\hline $\mathrm{N} 29$ & 1980 & 6,656 & & N79 & 1982 & 239,485 & & N104 & 1980 & 5,770 & \\
\hline $\mathrm{N} 29$ & 1981 & 16,523 & & N79 & 1983 & 533,069 & & N104 & 1981 & 2,490 & \\
\hline N29 & 1982 & 17,418 & & N79 & 1984 & 617,548 & & N104 & 1982 & 19,340 & \\
\hline $\mathrm{N} 29$ & 1983 & 15,390 & & N80 & 1980 & 54,956 & & N104 & 1983 & 5,360 & \\
\hline $\mathrm{N} 29$ & 1984 & 12,288 & & N80 & 1981 & 41,902 & & N104 & 1984 & 2,130 & \\
\hline N31 & 1980 & 162,434 & & N80 & 1982 & 9,299 & & N118 & 1980 & 3,726 & \\
\hline N31 & 1981 & 204,651 & & N80 & 1983 & 16,736 & & N118 & 1981 & 2,232 & \\
\hline N31 & 1982 & 14,787 & & N80 & 1984 & 47,148 & & N118 & 1982 & 6,354 & \\
\hline N31 & 1983 & 93,344 & & N81 & 1980 & 95,206 & & N118 & 1983 & 39,231 & \\
\hline N31 & 1984 & 8,945 & & N81 & 1981 & 87,506 & & N118 & 1984 & 5,804 & \\
\hline N37 & 1980 & 111,790 & & N81 & 1982 & 88,760 & & N119 & 1983 & 9,123 & \\
\hline N37 & 1981 & 74,540 & & N81 & 1983 & 145,791 & & N119 & 1984 & 500 & \\
\hline N37 & 1982 & 63,270 & & N81 & 1984 & 93,773 & & N132 & 1980 & 34,245 & \\
\hline N37 & 1983 & 56,830 & & N82 & 1980 & 111,987 & & N133 & 1980 & 137,196 & \\
\hline N37 & 1984 & 77,030 & & N82 & 1981 & 77,528 & & N133 & 1981 & 164,418 & \\
\hline N46 & 1980 & 27,459 & & N82 & 1982 & 86,499 & & N133 & 1982 & 127,860 & \\
\hline N46 & 1981 & 23,197 & & N82 & 1983 & 155,227 & & N133 & 1983 & 146,313 & \\
\hline N46 & 1982 & 27,174 & & N82 & 1984 & 102,183 & & N133 & 1984 & 151,649 & \\
\hline N46 & 1983 & 45,061 & & N83 & 1980 & 7,936 & & N134 & 1980 & 198,531 & \\
\hline N46 & 1984 & 65,064 & & N83 & 1981 & 129,479 & & N134 & 1981 & 224,447 & \\
\hline N68 & 1980 & 235,041 & & N83 & 1982 & 189,688 & & N134 & 1982 & 187,004 & \\
\hline N68 & 1981 & 218,780 & & N83 & 1983 & 189,836 & & N134 & 1983 & 240,846 & \\
\hline N68 & 1982 & 285,088 & & N83 & 1984 & 40,956 & & N134 & 1984 & 119,896 & \\
\hline N68 & 1983 & 241,412 & & N94 & 1980 & 24,024 & & N152 & 1980 & 44,541 & \\
\hline N68 & 1984 & 285,429 & & N94 & 1981 & 8,240 & & N152 & 1981 & 42,361 & \\
\hline N69 & 1980 & 263,832 & & N95 & 1980 & 120,496 & & N152 & 1982 & 44,913 & \\
\hline N69 & 1981 & 175,522 & & N95 & 1981 & 184,323 & & N152 & 1983 & 40,863 & \\
\hline N69 & 1982 & 280,297 & & N95 & 1982 & 185,041 & & N152 & 1984 & 62,471 & \\
\hline N69 & 1983 & 63,981 & & N95 & 1983 & 132,606 & & N198 & 1980 & 355,780 & \\
\hline N72 & 1980 & 53,122 & & N95 & 1984 & 114,901 & & N198 & 1981 & 226,480 & \\
\hline N72 & 1981 & 130,728 & & N97 & 1980 & 20,518 & & N198 & 1982 & 199,894 & \\
\hline N72 & 1982 & 174,957 & & N97 & 1981 & 36,327 & & N198 & 1983 & 335,700 & \\
\hline N72 & 1983 & 67,928 & & N97 & 1982 & 30,050 & & N198 & 1984 & 362,905 & \\
\hline
\end{tabular}


Table 2B. Public-supply pumpage in Kings, Queens, and Nassau Counties, N.Y., 1898-1984--continued

\begin{tabular}{|c|c|c|c|c|c|c|c|c|c|c|c|}
\hline $\begin{array}{c}\text { Well } \\
\text { number }\end{array}$ & Year & Pumpage & Remarks & $\begin{array}{c}\text { Well } \\
\text { number }\end{array}$ & Year & Pumpage & Remarks & $\begin{array}{c}\text { Well } \\
\text { number }\end{array}$ & Year & Pumpage & Remarks \\
\hline N199 & 1980 & 281,637 & & N1328 & 1982 & 292,760 & & $\mathrm{~N} 1802$ & 1980 & 34,059 & \\
\hline N199 & 1981 & 284,341 & & N1328 & 1983 & 248,201 & & N1802 & 1981 & 14,871 & \\
\hline N199 & 1982 & 180,867 & & N1328 & 1984 & 290,127 & & $\mathrm{~N} 1802$ & 1982 & 9,737 & \\
\hline N199 & 1983 & 394,023 & & N1402 & 1980 & 17 & & N1802 & 1983 & 74,283 & \\
\hline N199 & 1984 & 394,496 & & N1595 & 1981 & 1,125 & $\mathbf{M}$ & N1802 & 1984 & 48,187 & \\
\hline N570 & 1980 & 128,934 & & N1601 & 1980 & 354,286 & & $\mathrm{~N} 1870$ & 1980 & 995 & E \\
\hline N570 & 1981 & 252,061 & & N1601 & 1981 & 390,152 & & N1870 & 1981 & 183 & E \\
\hline N570 & 1982 & 218,062 & & N1601 & 1982 & 436,023 & & N1870 & 1982 & 1,065 & $E$ \\
\hline N570 & 1983 & 60,989 & & N1601 & 1983 & 398,470 & & N1870 & 1983 & 123 & $\mathbf{E}$ \\
\hline N570 & 1984 & 284,247 & & N1601 & 1984 & 336,186 & & N1870 & 1984 & 20,709 & $\infty$ \\
\hline N650 & 1981 & 4,802 & & $\mathrm{~N} 1602$ & 1980 & 252,922 & & N1871 & 1980 & 995 & $\mathrm{E}$ \\
\hline N650 & 1982 & 4,949 & & $\mathrm{~N} 1602$ & 1981 & 289,918 & & N1871 & 1981 & 183 & $\mathrm{E}$ \\
\hline N650 & 1984 & 721 & & N1602 & 1982 & 270,662 & & N1871 & 1982 & 1,065 & $\mathrm{E}$ \\
\hline N651 & 1981 & 3,191 & & N1602 & 1983 & 556,381 & & N1871 & 1983 & 123 & $\mathbf{E}$ \\
\hline N651 & 1982 & 3,443 & & $\mathrm{~N} 1602$ & 1984 & 430,402 & & N1871 & 1984 & 20,709 & $\mathrm{E}$ \\
\hline N651 & 1984 & 719 & & N1603 & 1980 & 493,124 & & N1872 & 1980 & 995 & $\mathbf{E}$ \\
\hline N687 & 1980 & 272,731 & & N1603 & 1981 & 596,620 & & N1872 & 1981 & 183 & $\mathrm{E}$ \\
\hline N687 & 1981 & 233,038 & & N1603 & 1982 & 483,741 & & N1872 & 1982 & 1,065 & $\mathrm{E}$ \\
\hline N687 & 1982 & 175,485 & & N1603 & 1983 & 368,551 & & N1872 & 1983 & 123 & $\mathrm{E}$ \\
\hline N687 & 1983 & 243,566 & & N1603 & 1984 & 629,295 & & N1872 & 1984 & 20,709 & $\mathrm{E}$ \\
\hline N687 & 1984 & 177,567 & & N1618 & 1980 & 220,896 & & N1873 & 1980 & 995 & $\mathbf{E}$ \\
\hline N693 & 1974 & 200 & & N1618 & 1981 & 251,265 & & N1873 & 1981 & 183 & $\mathrm{E}$ \\
\hline N693 & 1975 & 52,300 & & N1618 & 1982 & 217,712 & & N1873 & 1982 & 1,065 & E \\
\hline N693 & 1976 & 162,700 & & N1618 & 1983 & 401,071 & & N1873 & 1983 & 123 & $\mathrm{E}$ \\
\hline N693 & 1977 & 103,600 & & N1618 & 1984 & 371,701 & & N1873 & 1984 & 20,709 & E \\
\hline N693 & 1978 & 103,100 & & N1651 & 1980 & 58,944 & & N1874 & 1980 & 995 & $\mathrm{E}$ \\
\hline N693 & 1979 & 65,100 & & N1651 & 1981 & 40,819 & & N1874 & 1981 & 183 & $\mathrm{E}$ \\
\hline N693 & 1980 & 123,800 & & N1651 & 1982 & 12,456 & & N1874 & 1982 & 1,065 & $\mathrm{E}$ \\
\hline N693 & 1981 & 77,900 & & N1651 & 1983 & 107,308 & & N1874 & 1983 & 123 & $\mathrm{E}$ \\
\hline N693 & 1982 & 170,700 & & N1651 & 1984 & 22,151 & & N1874 & 1984 & 20,709 & $\mathrm{E}$ \\
\hline N693 & 1983 & 389,800 & & N1697 & 1980 & 174,947 & & $\mathrm{~N} 1875$ & 1980 & 995 & $\mathrm{E}$ \\
\hline N693 & 1984 & 363,300 & & N1697 & 1981 & 84,797 & & N1875 & 1981 & 183 & $\mathrm{E}$ \\
\hline N700 & 1980 & 20 & & N1697 & 1982 & 178,110 & & N1875 & 1982 & 1,065 & $\mathrm{E}$ \\
\hline N700 & 1982 & 1,155 & & N1697 & 1983 & 135,594 & & N1875 & 1983 & 123 & $\mathrm{E}$ \\
\hline N700 & 1983 & 849 & & N1697 & 1984 & 116,497 & & N1875 & 1984 & 20,709 & $\mathrm{E}$ \\
\hline N700 & 1984 & 41,979 & & $\mathrm{~N} 1715$ & 1980 & 94,350 & & N1876 & 1980 & 995 & $\mathrm{E}$ \\
\hline N835 & 1980 & 110,930 & & N1715 & 1981 & 98,350 & & N1876 & 1981 & 183 & $\mathrm{E}$ \\
\hline N835 & 1981 & 55,674 & & $\mathrm{~N} 1715$ & 1982 & 85,590 & & N1876 & 1982 & 1,065 & $\mathrm{E}$ \\
\hline N1298 & 1980 & 64,704 & & $\mathrm{~N} 1715$ & 1983 & 111,900 & & N1876 & 1983 & 123 & $\mathrm{E}$ \\
\hline N1298 & 1981 & 29,509 & & N1715 & 1984 & 116,620 & & N1876 & 1984 & 20,709 & $\mathrm{E}$ \\
\hline N1298 & 1982 & 90,180 & & N1716 & 1980 & 49,870 & & N1877 & 1980 & 949 & $\mathrm{E}$ \\
\hline N1298 & 1983 & 157,448 & & N1716 & 1981 & 60,470 & & N1877 & 1981 & 175 & $\mathrm{E}$ \\
\hline N1298 & 1984 & 269,800 & & N1716 & 1982 & 38,000 & & N1877 & 1982 & 1,017 & $\mathrm{E}$ \\
\hline N1328 & 1980 & 369,100 & & N1716 & 1983 & 63,940 & & N1877 & 1983 & 117 & $\mathrm{E}$ \\
\hline $\mathrm{N} 1328$ & 1981 & 286,875 & & N1716 & 1984 & 15,980 & & N1877 & 1984 & 19,768 & $\mathrm{E}$ \\
\hline
\end{tabular}


Table 2B. Public-supply pumpage in Kings, Queens, and Nassau Counties, N.Y., 1898-1984--continued

\begin{tabular}{|c|c|c|c|c|c|c|c|c|c|c|c|}
\hline $\begin{array}{c}\text { Well } \\
\text { number }\end{array}$ & Year & Pumpage & Remarks & $\begin{array}{c}\text { Well } \\
\text { number }\end{array}$ & Year & Pumpage & Remarks & $\begin{array}{c}\text { Well } \\
\text { number }\end{array}$ & Year & Pumpage & Remarks \\
\hline N1937 & 1980 & 10,640 & & N2214 & 1983 & 159,775 & & N2597 & 1980 & 320,807 & \\
\hline N1937 & 1981 & 5,052 & & $\mathrm{~N} 2214$ & 1984 & 129,538 & & N2597 & 1981 & 1,890 & \\
\hline N1937 & 1982 & 7,515 & & N2239 & 1980 & 335,698 & & N2597 & 1983 & 127,752 & \\
\hline N1937 & 1983 & 13,729 & & N2239 & 1981 & 319,186 & & N2597 & 1984 & 17,938 & \\
\hline N1937 & 1984 & 16,392 & & N2239 & 1982 & 185,259 & & $\mathrm{~N} 2602$ & 1980 & 97,638 & \\
\hline N1958 & 1974 & 436,400 & & N2239 & 1983 & 178,370 & & N2602 & 1981 & 102,623 & \\
\hline N1958 & 1975 & 495,000 & & $\mathrm{~N} 2239$ & 1984 & 245,136 & & $\mathrm{~N} 2602$ & 1982 & 149,607 & \\
\hline N1958 & 1976 & 476,200 & & N2400 & 1980 & 359,244 & & $\mathrm{~N} 2602$ & 1983 & 163,186 & \\
\hline N1958 & 1977 & 511,500 & & N2400 & 1981 & 196,589 & & $\mathrm{~N} 2602$ & 1984 & 154,594 & \\
\hline N1958 & 1978 & 420,200 & & $\mathrm{~N} 2400$ & 1982 & 120,422 & & $\mathrm{~N} 2613$ & 1980 & 17,168 & \\
\hline N1958 & 1979 & 613,700 & & $\mathrm{~N} 2400$ & 1983 & 125,199 & & $\mathrm{~N} 2613$ & 1981 & 17,982 & \\
\hline N1958 & 1980 & 386,700 & & $\mathrm{~N} 2400$ & 1984 & 367,339 & & N2613 & 1982 & 14,920 & \\
\hline N1958 & 1981 & 374,000 & & $\mathrm{~N} 2413$ & 1974 & 268,500 & & N2613 & 1983 & 24,521 & \\
\hline N1958 & 1982 & 617,500 & & $\mathrm{~N} 2413$ & 1975 & 398,800 & & N2613 & 1984 & 8,292 & \\
\hline N1958 & 1983 & 556,200 & & $\mathrm{~N} 2413$ & 1976 & 401,400 & & N2748 & 1980 & 134,691 & \\
\hline N1958 & 1984 & 569,700 & & $\mathrm{~N} 2413$ & 1977 & 367,500 & & N2748 & 1981 & 124,912 & \\
\hline N2028 & 1980 & 236,325 & & $\mathrm{~N} 2413$ & 1978 & 220,500 & & $\mathrm{~N} 2748$ & 1982 & 135,860 & \\
\hline N2028 & 1981 & 127,874 & & $\mathrm{~N} 2413$ & 1979 & 266,200 & & N2748 & 1983 & 156,184 & \\
\hline $\mathrm{N} 2028$ & 1982 & 103,368 & & $\mathrm{~N} 2413$ & 1980 & 131,400 & & N2748 & 1984 & 145,219 & \\
\hline N2028 & 1983 & 169,420 & & $\mathrm{~N} 2413$ & 1981 & 122,900 & & N2920 & 1981 & 600 & \\
\hline $\mathrm{N} 2028$ & 1984 & 214,426 & & $\mathrm{~N} 2413$ & 1982 & 116,800 & & N2920 & 1982 & 17,112 & \\
\hline N2030 & 1980 & 42,430 & & $\mathrm{~N} 2413$ & 1983 & 73,000 & & N2920 & 1983 & 18,000 & \\
\hline $\mathrm{N} 2030$ & 1981 & 47,770 & & $\mathrm{~N} 2413$ & 1984 & 14,400 & & N3185 & 1980 & 395,153 & \\
\hline N2030 & 1982 & 32,740 & & $\mathrm{~N} 2414$ & 1974 & 76,700 & & N3185 & 1981 & 232,276 & \\
\hline $\mathrm{N} 2030$ & 1983 & 49,970 & & N2414 & 1975 & 67,700 & & N3185 & 1982 & 306,274 & \\
\hline N2030 & 1984 & 10,220 & & $\mathrm{~N} 2414$ & 1976 & 74,400 & & N3185 & 1983 & 344,989 & \\
\hline N2052 & 1980 & 302,070 & & $\mathrm{~N} 2414$ & 1977 & 128,300 & & N3185 & 1984 & 116,016 & \\
\hline $\mathrm{N} 2052$ & 1981 & 284,850 & & $\mathrm{~N} 2414$ & 1978 & 196,800 & & N3427 & 1980 & 20,880 & \\
\hline $\mathrm{N} 2052$ & 1982 & 294,500 & & N2414 & 1979 & 30,900 & & N3427 & 1981 & 7,670 & \\
\hline N2052 & 1983 & 79,510 & & N2414 & 1980 & 78,300 & & N3443 & 1980 & 221,493 & \\
\hline $\mathrm{N} 2052$ & 1984 & 5,680 & & $\mathrm{~N} 2414$ & 1981 & 47,600 & & N3443 & 1981 & 248,041 & \\
\hline $\mathrm{N} 2115$ & 1974 & 192,700 & & N2414 & 1982 & 96,500 & & N3443 & 1982 & 269,446 & \\
\hline $\mathrm{N} 2115$ & 1975 & 24,300 & & $\mathrm{~N} 2414$ & 1983 & 92,200 & & N3443 & 1983 & 190,381 & \\
\hline $\mathrm{N} 2115$ & 1976 & 144,750 & & $\mathrm{~N} 2414$ & 1984 & 5,700 & & N3443 & 1984 & 291,387 & \\
\hline N2115 & 1977 & 111,300 & & N2565 & 1981 & 700 & & N3456 & 1980 & 29,926 & \\
\hline $\mathrm{N} 2115$ & 1978 & 95,200 & & $\mathrm{~N} 2578$ & 1980 & 687 & & N3456 & 1981 & 87,509 & \\
\hline $\mathrm{N} 2115$ & 1979 & 85,000 & & $\mathrm{~N} 2578$ & 1981 & 307 & & N3456 & 1982 & 239,661 & \\
\hline $\mathrm{N} 2115$ & 1980 & 86,100 & & $\mathrm{~N} 2578$ & 1982 & 273 & & N3456 & 1983 & 138,544 & \\
\hline $\mathrm{N} 2115$ & 1981 & 26,900 & & $\mathrm{~N} 2578$ & 1983 & 67 & & N3456 & 1984 & 207,849 & \\
\hline N2115 & 1982 & 39,800 & & $\mathrm{~N} 2578$ & 1984 & 94 & & N3457 & 1980 & 31,805 & \\
\hline $\mathrm{N} 2115$ & 1983 & 90,700 & & $\mathrm{~N} 2580$ & 1980 & 35,450 & & N3457 & 1981 & 8,013 & \\
\hline $\mathrm{N} 2115$ & 1984 & 45,300 & & $\mathrm{~N} 2580$ & 1981 & 8,382 & & N3457 & 1982 & 3,803 & \\
\hline N2214 & 1980 & 84,529 & & N2580 & 1982 & 2,976 & & N3457 & 1983 & 3,587 & \\
\hline $\mathrm{N} 2214$ & 1981 & 73,783 & & $\mathrm{~N} 2580$ & 1983 & 16,464 & & N3457 & 1984 & 18,517 & \\
\hline $\mathrm{N} 2214$ & 1982 & 249,547 & & $\mathrm{~N} 2580$ & 1984 & 4,504 & & N3465 & 1980 & 327,286 & \\
\hline
\end{tabular}


Table 2B. Public-supply pumpage in Kings, Queens, and Nassau Counties, N.Y., 1898-1984--continued

\begin{tabular}{|c|c|c|c|c|c|c|c|c|c|c|c|}
\hline $\begin{array}{c}\text { Well } \\
\text { number }\end{array}$ & Year & Pumpage & Remarks & $\begin{array}{c}\text { Well } \\
\text { number }\end{array}$ & Year & Pumpage & Remarks & $\begin{array}{c}\text { Well } \\
\text { number }\end{array}$ & Year & Pumpage & Remarks \\
\hline N3465 & 1981 & 220,727 & & N3618 & 1983 & 176,010 & & N3733 & 1981 & 107,762 & \\
\hline N3465 & 1982 & 282,855 & & N3618 & 1984 & 172,528 & & N3733 & 1982 & 40,285 & \\
\hline N3465 & 1983 & 365,520 & & N3668 & 1980 & 95,559 & & N3733 & 1983 & 41,761 & \\
\hline N3465 & 1984 & 363,678 & & N3668 & 1981 & 379,200 & & N3733 & 1984 & 38,426 & \\
\hline N3474 & 1980 & 150,165 & & N3668 & 1982 & 370,165 & & N3745 & 1980 & 197,149 & \\
\hline N3474 & 1981 & 136,625 & & N3668 & 1983 & 365,624 & & N3745 & 1981 & 157,541 & \\
\hline N3474 & 1982 & 132,695 & & N3668 & 1984 & 430,983 & & N3745 & 1982 & 114,239 & \\
\hline N3474 & 1983 & 146,493 & & N3672 & 1980 & 181,337 & & N3745 & 1983 & 129,716 & \\
\hline N3474 & 1984 & 150,793 & & N3672 & 1981 & 45,181 & & N3745 & 1984 & 64,797 & \\
\hline N3475 & 1980 & 162,684 & & N3672 & 1982 & 350 & & N3781 & 1980 & 728,499 & \\
\hline N3475 & 1981 & 170,117 & & N3680 & 1980 & 160,150 & & N3781 & 1981 & 602,983 & \\
\hline N3475 & 1982 & 199,521 & & N3680 & 1981 & 20,740 & & N3781 & 1982 & 689,009 & \\
\hline N3475 & 1983 & 217,460 & & N3680 & 1982 & 4,130 & & N3781 & 1983 & 626,875 & \\
\hline N3475 & 1984 & 138,172 & & N3687 & 1980 & 95,772 & & N3781 & 1984 & 668,102 & \\
\hline N3520 & 1980 & 22,033 & & N3687 & 1981 & 310,992 & & N3832 & 1980 & 412 & \\
\hline N3520 & 1981 & 25,389 & & N3687 & 1982 & 226,520 & & N3832 & 1981 & 2,170 & \\
\hline N3520 & 1982 & 18,708 & & N3687 & 1983 & 139,153 & & N3832 & 1982 & 500 & \\
\hline N3520 & 1983 & 21,773 & & N3687 & 1984 & 253,627 & & N3832 & 1983 & 4,706 & \\
\hline N3520 & 1984 & 7,888 & & N3704 & 1980 & 305,319 & & N3832 & 1984 & 608 & \\
\hline N3523 & 1980 & 40,519 & & N3704 & 1981 & 264,856 & & N3878 & 1980 & 164,794 & \\
\hline N3523 & 1983 & 240,105 & & N3704 & 1982 & 128,902 & & N3878 & 1981 & 13,083 & \\
\hline N3523 & 1984 & 359,011 & & N3704 & 1983 & 291,957 & & N3878 & 1982 & 15,432 & \\
\hline N3540 & 1980 & 64,634 & & N3704 & 1984 & 229,189 & & N3878 & 1983 & 57,325 & \\
\hline N3540 & 1981 & 53,844 & & N3720 & 1974 & 184,700 & & N3878 & 1984 & 46,015 & \\
\hline N3540 & 1982 & 39,873 & & N3720 & 1975 & 366,800 & & N3881 & 1980 & 207,242 & \\
\hline N3540 & 1983 & 55,202 & & N3720 & 1976 & 217,300 & & N3881 & 1981 & 189,784 & \\
\hline N3540 & 1984 & 50,336 & & N3720 & 1977 & 127,100 & & N3881 & 1982 & 189,430 & \\
\hline N3603 & 1980 & 90,949 & & N3720 & 1978 & 94,300 & & N3881 & 1983 & 204,035 & \\
\hline N3603 & 1981 & 67,789 & & N3720 & 1979 & 73,100 & & N3881 & 1984 & 212,869 & \\
\hline N3603 & 1982 & 64,198 & & N3720 & 1980 & 156,000 & & N3893 & 1980 & 36,160 & \\
\hline N3603 & 1983 & 83,044 & & N3720 & 1981 & 159,000 & & N3893 & 1981 & 7,420 & \\
\hline N3603 & 1984 & 43,054 & & N3720 & 1982 & 159,700 & & N3893 & 1982 & 7,040 & \\
\hline N3604 & 1980 & 48,793 & & N3720 & 1983 & 357,100 & & N3893 & 1983 & 12,640 & \\
\hline N3604 & 1981 & 53,809 & & N3720 & 1984 & 329,500 & & N3894 & 1980 & 32,090 & \\
\hline N3604 & 1982 & 62,301 & & N3722 & 1980 & 51 & & N3894 & 1981 & 5,190 & \\
\hline N3604 & 1983 & 39,948 & & N3722 & 1981 & 166 & & N3894 & 1982 & 1,270 & \\
\hline N3604 & 1984 & 38,569 & & N3722 & 1982 & 60 & & N3895 & 1980 & 745,010 & \\
\hline N3605 & 1980 & 13,358 & & N3722 & 1983 & 183 & & N3895 & 1981 & 478,900 & \\
\hline N3605 & 1981 & 3,275 & & N3722 & 1984 & 235 & & N3895 & 1982 & 248,150 & \\
\hline N3605 & 1982 & 6,738 & & N3732 & 1980 & 69,591 & & N3895 & 1983 & 91,190 & \\
\hline N3605 & 1983 & 21,804 & & N3732 & 1981 & 89,624 & & N3895 & 1984 & 27,080 & \\
\hline N3605 & 1984 & 240 & & N3732 & 1982 & 105,374 & & N3905 & 1980 & 14,692 & \\
\hline N3618 & 1980 & 60,000 & & N3732 & 1983 & 113,510 & & N3905 & 1983 & 33,480 & \\
\hline N3618 & 1981 & 69,120 & & N3732 & 1984 & 135,939 & & N3934 & 1980 & 250,071 & \\
\hline N3618 & 1982 & 57,004 & & N3733 & 1980 & 38,729 & & N3934 & 1981 & 233,097 & \\
\hline
\end{tabular}


Table 2B. Public-supply pumpage in Kings, Queens, and Nassau Counties, N.Y., 1898-1984--continued

\begin{tabular}{|c|c|c|c|c|c|c|c|c|c|c|c|}
\hline $\begin{array}{c}\text { Well } \\
\text { number }\end{array}$ & Year & Pumpage & Remarks & $\begin{array}{c}\text { Well } \\
\text { number }\end{array}$ & Year & Pumpage & Remarks & $\begin{array}{c}\text { Well } \\
\text { number }\end{array}$ & Year & Pumpage & Remarks \\
\hline N3934 & 1982 & 208,201 & & N4096 & 1981 & 209,427 & & N4298 & 1981 & 483,000 & \\
\hline N3934 & 1983 & 256,043 & & N4096 & 1982 & 145,434 & & N4298 & 1982 & 622,400 & \\
\hline N3934 & 1984 & 260,488 & & N4096 & 1983 & 162,317 & & N4298 & 1983 & 724,800 & \\
\hline N3935 & 1980 & 295,800 & & N4096 & 1984 & 39,835 & & N4298 & 1984 & 318,200 & \\
\hline N3935 & 1981 & 244,763 & & N4097 & 1980 & 134,957 & & $\mathrm{~N} 4327$ & 1980 & 98,140 & \\
\hline N3935 & 1982 & 241,910 & & N4097 & 1981 & 131,167 & & N4327 & 1981 & 97,392 & \\
\hline N3935 & 1983 & 271,427 & & N4097 & 1982 & 119,497 & & $\mathrm{~N} 4327$ & 1982 & 66,911 & \\
\hline N3935 & 1984 & 243,954 & & N4097 & 1983 & 170,795 & & N4327 & 1983 & 98,429 & \\
\hline N3937 & 1980 & 374,897 & & N4097 & 1984 & 119,693 & & N4327 & 1984 & 60,315 & \\
\hline N3937 & 1981 & 293,468 & & N4118 & 1980 & 303,593 & & N4388 & 1980 & 503,671 & \\
\hline N3937 & 1982 & 347,758 & & N4118 & 1981 & 288,406 & & N4388 & 1981 & 497,149 & \\
\hline N3937 & 1983 & 255,308 & & N4118 & 1982 & 258,007 & & N4388 & 1982 & 468,862 & \\
\hline N3937 & 1984 & 229,071 & & N4118 & 1983 & 316,731 & & N4388 & 1983 & 223,401 & \\
\hline N3953 & 1980 & 26,049 & & N4118 & 1984 & 262,394 & & N4388 & 1984 & 76,148 & \\
\hline N3953 & 1981 & 5,023 & & $\mathrm{~N} 4132$ & 1980 & 293,574 & & N4389 & 1980 & 58,740 & \\
\hline N3953 & 1982 & 8,321 & & N4132 & 1981 & 281,594 & & N4389 & 1981 & 48,740 & \\
\hline N3953 & 1983 & 37,600 & & N4132 & 1982 & 211,248 & & N4389 & 1982 & 49,990 & \\
\hline N3953 & 1984 & 5,115 & & N4132 & 1983 & 298,278 & & N4389 & 1983 & 64,090 & \\
\hline N4043 & 1980 & 85,642 & & N4132 & 1984 & 208,773 & & N4389 & 1984 & 41,692 & \\
\hline N4043 & 1981 & 78,185 & & N4206 & 1980 & 103,347 & & N4390 & 1974 & 572,700 & \\
\hline N4043 & 1982 & 13,379 & & N4206 & 1981 & 113,044 & & N4390 & 1975 & 280,100 & \\
\hline N4043 & 1983 & 284,557 & & N4206 & 1982 & 122,166 & & N4390 & 1976 & 423,900 & \\
\hline N4043 & 1984 & 290,022 & & N4206 & 1983 & 126,644 & & N4390 & 1977 & 644,000 & \\
\hline N4077 & 1974 & 33,700 & & N4206 & 1984 & 106,039 & & N4390 & 1978 & 652,100 & \\
\hline N4077 & 1975 & 15,500 & & $\mathrm{~N} 4223$ & 1980 & 206,560 & & $\mathrm{~N} 4390$ & 1979 & 647,800 & \\
\hline N4077 & 1976 & 25,300 & & $\mathrm{~N} 4223$ & 1981 & 84,650 & & N4390 & 1980 & 656,800 & \\
\hline N4077 & 1977 & 30,000 & & N4243 & 1980 & 50,767 & & N4390 & 1981 & 582,700 & \\
\hline N4077 & 1978 & 30,400 & & N4243 & 1983 & 22,509 & & N4390 & 1982 & 648,700 & \\
\hline N4077 & 1979 & 19,600 & & N4245 & 1980 & 466,717 & & N4390 & 1983 & 622,500 & \\
\hline N4077 & 1980 & 37,600 & & N4245 & 1981 & 247,151 & & N4390 & 1984 & 555,500 & \\
\hline N4077 & 1981 & 45,300 & & N4245 & 1982 & 272,201 & & N4393 & 1980 & 237,893 & \\
\hline N4077 & 1982 & 25,900 & & N4245 & 1983 & 335,501 & & N4393 & 1981 & 303,548 & \\
\hline N4077 & 1983 & 88,900 & & N4245 & 1984 & 245,521 & & $\mathrm{~N} 4393$ & 1982 & 239,112 & \\
\hline N4077 & 1984 & 160,700 & & N4265 & 1980 & 19,663 & & N4393 & 1983 & 297,110 & \\
\hline N4082 & 1980 & 69,006 & & N4265 & 1981 & 18,752 & & N4393 & 1984 & 203,642 & \\
\hline N4082 & 1981 & 115,431 & & N4265 & 1982 & 28,921 & & N4400 & 1980 & 242,807 & \\
\hline $\mathrm{N} 4082$ & 1982 & 95,573 & & N4265 & 1983 & 39,184 & & N4400 & 1981 & 235,020 & \\
\hline $\mathrm{N} 4082$ & 1983 & 121,641 & & $\mathrm{~N} 4265$ & 1984 & 18,509 & & N4400 & 1982 & 208,854 & \\
\hline $\mathrm{N} 4082$ & 1984 & 194,290 & & N4298 & 1974 & 840,800 & & $\mathrm{~N} 4400$ & 1983 & 211,239 & \\
\hline N4095 & 1980 & 149,895 & & N4298 & 1975 & 603,100 & & N4400 & 1984 & 229,503 & \\
\hline N4095 & 1981 & 154,737 & & N4298 & 1976 & 553,000 & & N4405 & 1980 & 84,248 & \\
\hline N4095 & 1982 & 176,025 & & N4298 & 1977 & 716,100 & & N4405 & 1981 & 53,674 & \\
\hline N4095 & 1983 & 146,670 & & N4298 & 1978 & 721,300 & & N4405 & 1982 & 57,945 & \\
\hline N4095 & 1984 & 240,971 & & N4298 & 1979 & 653,100 & & N4405 & 1983 & 97,094 & \\
\hline N4096 & 1980 & 259,271 & & N4298 & 1980 & 604,000 & & N4405 & 1984 & 57,502 & \\
\hline
\end{tabular}


Table 2B. Public-supply pumpage in Kings, Queens, and Nassau Counties, N.Y., 1898-1984--continued

\begin{tabular}{|c|c|c|c|c|c|c|c|c|c|c|c|}
\hline $\begin{array}{c}\text { Well } \\
\text { number }\end{array}$ & Year & Pumpage & Remarks & $\begin{array}{c}\text { Well } \\
\text { number }\end{array}$ & Year & Pumpage & Remarks & $\begin{array}{c}\text { Well } \\
\text { number }\end{array}$ & Year & Pumpage & Remarks \\
\hline N4411 & 1980 & 106,919 & & N4602 & 1982 & 68,381 & & N5145 & 1980 & 270,283 & \\
\hline N4411 & 1981 & 112,282 & & N4602 & 1983 & 204,106 & & N5145 & 1981 & 92,174 & \\
\hline N4411 & 1982 & 127,207 & & N4602 & 1984 & 241,483 & & N5145 & 1982 & 114,190 & \\
\hline N4411 & 1983 & 113,276 & & N4623 & 1980 & 136,956 & & N5145 & 1983 & 79,267 & \\
\hline N4411 & 1984 & 118,418 & & N4623 & 1981 & 60,287 & & N5145 & 1984 & 51,123 & \\
\hline N4425 & 1980 & 483,092 & & N4623 & 1982 & 97,563 & & N5147 & 1980 & 69,437 & E \\
\hline N4425 & 1981 & 391,789 & & N4623 & 1983 & 60,150 & & N5147 & 1981 & 72,607 & E \\
\hline N4425 & 1982 & 347,717 & & N4623 & 1984 & 8,366 & & N5147 & 1982 & 43,053 & $\mathrm{E}$ \\
\hline N4425 & 1983 & 248,404 & & N4756 & 1980 & 56,271 & & N5147 & 1983 & 27,507 & $\mathrm{E}$ \\
\hline N4425 & 1984 & 204,902 & & N4756 & 1981 & 42,181 & & N5147 & 1984 & 12,800 & $\mathrm{E}$ \\
\hline N4447 & 1980 & 49,671 & & N4756 & 1982 & 17,058 & & N5148 & 1980 & 14,006 & \\
\hline N4447 & 1981 & 12,055 & & N4756 & 1983 & 2,040 & & N5148 & 1981 & 5,176 & \\
\hline N4447 & 1982 & 8,171 & & N4757 & 1980 & 265,878 & & N5148 & 1982 & 20,738 & \\
\hline N4447 & 1983 & 30,120 & & N4757 & 1981 & 281,438 & & N5148 & 1983 & 26,130 & \\
\hline N4448 & 1980 & 414,371 & & N4757 & 1982 & 364,376 & & N5148 & 1984 & 6,015 & \\
\hline N4448 & 1981 & 254,307 & & N4757 & 1983 & 391,332 & & N5152 & 1980 & 279,436 & \\
\hline N4448 & 1982 & 409,615 & & N4757 & 1984 & 395,573 & & N5152 & 1981 & 81,073 & \\
\hline N4448 & 1983 & 387,171 & & N4758 & 1980 & 59,837 & & N5152 & 1982 & 150,628 & \\
\hline N4448 & 1984 & 433,105 & & N4758 & 1981 & 343,377 & & N5152 & 1983 & 213,211 & \\
\hline N4450 & 1980 & 147,878 & & N4758 & 1982 & 384,298 & & N5152 & 1984 & 67,236 & \\
\hline N4450 & 1981 & 139,325 & & N4758 & 1983 & 350,896 & & N5153 & 1980 & 41,156 & \\
\hline N4450 & 1982 & 132,273 & & N4758 & 1984 & 252,850 & & N5153 & 1981 & 19,933 & \\
\hline N4450 & 1983 & 300,663 & & N4759 & 1980 & 285,366 & & N5153 & 1982 & 43,105 & \\
\hline N4450 & 1984 & 181,742 & & N4759 & 1981 & 19,518 & & N5153 & 1983 & 42,660 & \\
\hline N4451 & 1980 & 121,895 & & N4859 & 1982 & 130 & & N5153 & 1984 & 12,395 & \\
\hline N4451 & 1981 & 72,894 & & N4859 & 1983 & 6,380 & & N5155 & 1974 & 20,200 & \\
\hline N4451 & 1982 & 129,958 & & N4859 & 1984 & 3,810 & & N5155 & 1975 & 20,700 & \\
\hline $\mathrm{N} 4451$ & 1983 & 241,314 & & N4860 & 1980 & 21,680 & & N5155 & 1976 & 33,400 & \\
\hline N4451 & 1984 & 197,979 & & N4860 & 1981 & 19,950 & & N5155 & 1977 & 39,500 & \\
\hline N4461 & 1980 & 77,830 & & N4860 & 1982 & 17,670 & & N5155 & 1978 & 48,100 & \\
\hline N4461 & 1981 & 16,840 & & N4860 & 1983 & 21,980 & & N5155 & 1979 & 30,900 & \\
\hline N4461 & 1982 & 17,850 & & N4860 & 1984 & 20,320 & & N5155 & 1980 & 52,800 & \\
\hline $\mathrm{N} 4512$ & 1974 & 837,500 & & N5007 & 1982 & 78,712 & & N5155 & 1981 & 21,300 & \\
\hline $\mathrm{N} 4512$ & 1975 & 568,100 & & N5007 & 1983 & 190,639 & & N5155 & 1982 & 12,400 & \\
\hline N4512 & 1976 & 608,500 & & N5007 & 1984 & 161,627 & & N5155 & 1983 & 25,100 & \\
\hline $\mathrm{N} 4512$ & 1977 & 720,300 & & N5099 & 1980 & 416,038 & & N5155 & 1984 & 5,630 & \\
\hline N4512 & 1978 & 654,200 & & N5099 & 1981 & 433,808 & & N5156 & 1974 & 763,500 & \\
\hline $\mathrm{N} 4512$ & 1979 & 452,800 & & N5099 & 1982 & 327,775 & & N5156 & 1975 & 735,000 & \\
\hline $\mathrm{N} 4512$ & 1980 & 555,700 & & N5099 & 1983 & 372,558 & & N5156 & 1976 & 788,300 & \\
\hline $\mathrm{N} 4512$ & 1981 & 656,800 & & N5099 & 1984 & 310,852 & & N5156 & 1977 & 739,500 & \\
\hline $\mathrm{N} 4512$ & 1982 & 654,600 & & N5121 & 1980 & 432,672 & & N5156 & 1978 & 733,200 & \\
\hline $\mathrm{N} 4512$ & 1983 & 629,100 & & N5121 & 1981 & 708,823 & & N5156 & 1979 & 797,200 & \\
\hline $\mathrm{N} 4512$ & 1984 & 213,000 & & N5121 & 1982 & 562,411 & & N5156 & 1980 & 680,800 & \\
\hline $\mathrm{N} 4602$ & 1980 & 57,728 & & N5121 & 1983 & 488,943 & & N5156 & 1981 & 438,400 & \\
\hline $\mathrm{N} 4602$ & 1981 & 52,922 & & N5121 & 1984 & 324,865 & & N5156 & 1982 & 530,600 & \\
\hline
\end{tabular}


Table 2B. Public-supply pumpage in Kings, Queens, and Nassau Counties, N.Y., 1898-1984--continued

\begin{tabular}{|c|c|c|c|c|c|c|c|c|c|c|c|}
\hline $\begin{array}{c}\text { Well } \\
\text { number }\end{array}$ & Year & Pumpage & Remarks & $\begin{array}{c}\text { Well } \\
\text { number }\end{array}$ & Year & Pumpage & Remarks & $\begin{array}{c}\text { Well } \\
\text { number }\end{array}$ & Year & Pumpage & Remarks \\
\hline N5156 & 1983 & 402,300 & & N5260 & 1983 & 121,248 & & N5528 & 1981 & 131,267 & \\
\hline N5156 & 1984 & 689,400 & & N5260 & 1984 & 60,800 & & N5528 & 1982 & 156,506 & \\
\hline N5163 & 1980 & 224,197 & & N5302 & 1980 & 58,424 & & N5528 & 1983 & 130,574 & \\
\hline N5163 & 1981 & 229,942 & & N5302 & 1981 & 59,481 & & N5528 & 1984 & 127,977 & \\
\hline N5163 & 1982 & 208,662 & & N5302 & 1982 & 220,069 & & N5596 & 1980 & 178,269 & \\
\hline N5163 & 1983 & 249,026 & & N5302 & 1983 & 171,441 & & N5596 & 1981 & 212,238 & \\
\hline N5163 & 1984 & 212,390 & & N5302 & 1984 & 253,317 & & N5596 & 1982 & 224,696 & \\
\hline N5187 & 1980 & 256,307 & & N5303 & 1980 & 199,726 & & N5596 & 1983 & 219,869 & \\
\hline N5187 & 1981 & 246,689 & & N5303 & 1981 & 477,247 & & N5596 & 1984 & 273,846 & \\
\hline N5187 & 1982 & 200,822 & & N5308 & 1981 & 34,660 & & N5603 & 1980 & 332,866 & \\
\hline N5187 & 1983 & 320,990 & & N5308 & 1982 & 24,378 & & N5603 & 1981 & 203,060 & \\
\hline N5187 & 1984 & 474,914 & & N5308 & 1983 & 395,228 & & N5653 & 1980 & 322,328 & \\
\hline N5193 & 1980 & 130,067 & & N5308 & 1984 & 215,612 & & N5653 & 1981 & 377,307 & \\
\hline N5193 & 1981 & 18,163 & & N5318 & 1980 & 139,182 & & N5653 & 1982 & 479,968 & \\
\hline N5193 & 1982 & 68,801 & & N5318 & 1981 & 90,202 & & N5653 & 1983 & 245,588 & \\
\hline N5193 & 1983 & 140,695 & & N5318 & 1982 & 68,463 & & N5653 & 1984 & 328,240 & \\
\hline N5193 & 1984 & 165,449 & & N5318 & 1983 & 337,797 & & N5655 & 1980 & 97,793 & $\mathrm{E}$ \\
\hline N5194 & 1980 & 332,394 & & N5318 & 1984 & 139,971 & & N5655 & 1981 & 82,124 & $\mathrm{E}$ \\
\hline N5194 & 1981 & 416,424 & & N5319 & 1980 & 1,134 & & N5655 & 1982 & 35,956 & $\mathrm{E}$ \\
\hline N5194 & 1982 & 190,724 & & N5320 & 1980 & 104,308 & & N5655 & 1983 & 30,283 & $E$ \\
\hline N5194 & 1983 & 166,409 & & N5320 & 1981 & 74,582 & & N5655 & 1984 & 23,431 & $E$ \\
\hline N5194 & 1984 & 216,183 & & N5320 & 1982 & 39,876 & & N5656 & 1980 & 293,342 & \\
\hline N5195 & 1980 & 111,295 & & N5320 & 1983 & 291,156 & & N5656 & 1981 & 167,299 & \\
\hline N5195 & 1981 & 154,800 & & N5320 & 1984 & 388,986 & & N5656 & 1982 & 233,922 & \\
\hline N5195 & 1982 & 210,834 & & N5321 & 1980 & 114,634 & & N5656 & 1983 & 371,171 & \\
\hline N5195 & 1983 & 200,470 & & N5321 & 1981 & 153,778 & & N5656 & 1984 & 296,865 & \\
\hline N5195 & 1984 & 110,722 & & N5321 & 1982 & 79,279 & & N5695 & 1980 & 294,356 & \\
\hline N5201 & 1980 & 12,889 & & N5321 & 1983 & 154,002 & & N5695 & 1981 & 249,298 & \\
\hline N5201 & 1983 & 17,226 & & N5321 & 1984 & 124,490 & & N5695 & 1982 & 194,085 & \\
\hline N5209 & 1980 & 49,180 & & N5322 & 1980 & 106,280 & & N5695 & 1983 & 223,915 & \\
\hline N5209 & 1981 & 65,070 & & N5322 & 1981 & 122,526 & & N5695 & 1984 & 363,375 & \\
\hline N5209 & 1982 & 42,990 & & N5322 & 1982 & 34,421 & & N5696 & 1980 & 127,318 & \\
\hline N5209 & 1983 & 82,550 & & N5322 & 1983 & 61,812 & & N5696 & 1981 & 174,210 & \\
\hline N5209 & 1984 & 129,780 & & N5322 & 1984 & 36,549 & & N5696 & 1982 & 268,807 & \\
\hline N5227 & 1980 & 200,415 & & N5484 & 1980 & 245,910 & & N5696 & 1983 & 362,900 & \\
\hline N5227 & 1981 & 144,410 & & N5484 & 1981 & 283,493 & & N5696 & 1984 & 285,945 & \\
\hline N5227 & 1982 & 173,447 & & N5484 & 1982 & 185,870 & & N5703 & 1980 & 143,918 & \\
\hline N5227 & 1983 & 115,751 & & N5484 & 1983 & 64,068 & & N5703 & 1981 & 279,862 & \\
\hline N5227 & 1984 & 86,774 & & N5484 & 1984 & 131,961 & & N5703 & 1982 & 297,361 & \\
\hline N5259 & 1980 & 143,450 & & N5485 & 1980 & 66,387 & & N5703 & 1983 & 209,981 & \\
\hline N5259 & 1981 & 97,700 & & N5485 & 1981 & 65,323 & & N5703 & 1984 & 485,498 & \\
\hline N5259 & 1982 & 5,780 & & N5485 & 1982 & 158,786 & & N5710 & 1980 & 305,267 & \\
\hline N5260 & 1980 & 69,377 & & N5485 & 1983 & 52,739 & & N5710 & 1981 & 344,461 & \\
\hline N5260 & 1981 & 59,066 & & N5485 & 1984 & 76,562 & & N5710 & 1982 & 383,846 & \\
\hline N5260 & 1982 & 181,300 & & N5528 & 1980 & 197,817 & & N5710 & 1983 & 137,686 & \\
\hline
\end{tabular}


Table 2B. Public-supply pumpage in Kings, Queens, and Nassau Counties, N.Y., 1898-1984--continued

\begin{tabular}{|c|c|c|c|c|c|c|c|c|c|c|c|}
\hline $\begin{array}{c}\text { Well } \\
\text { number }\end{array}$ & Year & Pumpage & Remarks & $\begin{array}{c}\text { Well } \\
\text { number }\end{array}$ & Year & Pumpage & Remarks & $\begin{array}{c}\text { Well } \\
\text { number }\end{array}$ & Year & Pumpage & Remarks \\
\hline N5710 & 1984 & 6,062 & & N6076 & 1984 & 206,794 & & N6150 & 1984 & 222,062 & \\
\hline N5762 & 1980 & 529,170 & & N6077 & 1980 & 120,497 & & N6190 & 1980 & 17,170 & \\
\hline N5762 & 1981 & 277,544 & & N6077 & 1981 & 71,303 & & N6190 & 1981 & 2,336 & \\
\hline N5762 & 1982 & 210,059 & & N6077 & 1982 & 169,450 & & N6190 & 1982 & 931 & \\
\hline N5762 & 1983 & 293,511 & & N6077 & 1983 & 141,121 & & N6190 & 1983 & 766 & \\
\hline N5762 & 1984 & 210,389 & & N6077 & 1984 & 197,722 & & N6191 & 1980 & 4,778 & \\
\hline N5767 & 1980 & 424,060 & & N6078 & 1980 & 31,465 & & N6192 & 1980 & 128,720 & \\
\hline N5767 & 1981 & 534,750 & & N6078 & 1981 & 21,311 & & N6192 & 1981 & 24,197 & \\
\hline N5767 & 1982 & 546,500 & & N6078 & 1982 & 14,501 & & N6192 & 1982 & 50,543 & \\
\hline N5767 & 1983 & 332,090 & & N6078 & 1983 & 29,810 & & N6192 & 1983 & 44,013 & \\
\hline N5767 & 1984 & 292,030 & & N6078 & 1984 & 3,221 & & N6192 & 1984 & 28,502 & \\
\hline N5792 & 1980 & 382,925 & & N6087 & 1980 & 21,370 & & N6315 & 1980 & 106,617 & \\
\hline N5792 & 1981 & 377,088 & & N6087 & 1981 & 22,660 & & N6315 & 1981 & 114,905 & \\
\hline N5792 & 1982 & 397,839 & & N6087 & 1982 & 1,050 & & N6315 & 1982 & 133,449 & \\
\hline N5792 & 1983 & 399,477 & & N6087 & 1983 & 22,410 & & N6315 & 1983 & 135,892 & \\
\hline N5792 & 1984 & 297,396 & & N6087 & 1984 & 21,610 & & N6315 & 1984 & 125,447 & \\
\hline N5852 & 1980 & 298,241 & & N6092 & 1980 & 231,149 & & N6442 & 1980 & 81,143 & \\
\hline N5852 & 1981 & 355,240 & & N6092 & 1981 & 233,237 & & N6442 & 1981 & 249,761 & \\
\hline N5852 & 1982 & 536,217 & & N6092 & 1982 & 280,453 & & N6442 & 1982 & 90,401 & \\
\hline N5852 & 1983 & 474,631 & & N6092 & 1983 & 316,710 & & N6442 & 1983 & 82,464 & \\
\hline N5852 & 1984 & 421,898 & & N6092 & 1984 & 315,141 & & N6442 & 1984 & 49,414 & \\
\hline N5876 & 1980 & 50,570 & & N6093 & 1980 & 224,329 & & N6443 & 1980 & 38,717 & \\
\hline N5876 & 1981 & 31,720 & & N6093 & 1981 & 143,681 & & N6443 & 1981 & 39,509 & \\
\hline N5876 & 1982 & 56,280 & & N6093 & 1982 & 168,116 & & N6443 & 1982 & 23,613 & \\
\hline N5876 & 1983 & 103,930 & & N6093 & 1983 & 151,280 & & N6443 & 1983 & 38,345 & \\
\hline N5876 & 1984 & 93,800 & & N6093 & 1984 & 105,800 & & N6443 & 1984 & 9,629 & \\
\hline N5884 & 1980 & 293,761 & & N6146 & 1980 & 71,223 & & N6450 & 1980 & 353,994 & \\
\hline N5884 & 1981 & 241,117 & & N6146 & 1981 & 54,245 & & N6450 & 1981 & 401,446 & \\
\hline N5884 & 1982 & 235,054 & & N6146 & 1982 & 49,099 & & N6450 & 1982 & 90,440 & \\
\hline N5884 & 1983 & 308,329 & & N6146 & 1983 & 54,295 & & N6450 & 1984 & 41,287 & \\
\hline N5884 & 1984 & 229,912 & & N6146 & 1984 & 109,506 & & N6580 & 1980 & 154,181 & \\
\hline N5947 & 1980 & 302,731 & & N6148 & 1980 & 214,940 & & N6580 & 1981 & 125,574 & \\
\hline N5947 & 1981 & 217,096 & & N6148 & 1981 & 135,040 & & N6580 & 1982 & 153,743 & \\
\hline N5947 & 1982 & 243,100 & & N6148 & 1982 & 233,613 & & N6580 & 1983 & 148,009 & \\
\hline N5947 & 1983 & 187,525 & & N6148 & 1983 & 232,953 & & N6580 & 1984 & 91,651 & \\
\hline N5947 & 1984 & 223,478 & & N6148 & 1984 & 204,015 & & N6644 & 1980 & 172,877 & \\
\hline N6045 & 1980 & 82,680 & & N6149 & 1980 & 69,437 & $\mathrm{E}$ & N6644 & 1981 & 171,249 & \\
\hline N6045 & 1981 & 69,473 & & N6149 & 1981 & 72,607 & $\mathrm{E}$ & N6644 & 1982 & 170,681 & \\
\hline N6045 & 1982 & 36,028 & & N6149 & 1982 & 43,053 & $\mathrm{E}$ & N6644 & 1983 & 175,192 & \\
\hline N6045 & 1983 & 65,964 & & N6149 & 1983 & 27,507 & $\mathbf{E}$ & N6644 & 1984 & 153,272 & \\
\hline N6045 & 1984 & 66,128 & & N6149 & 1984 & 12,801 & $\mathrm{E}$ & N6651 & 1980 & 214,734 & \\
\hline N6076 & 1980 & 107,230 & & N6150 & 1980 & 238,537 & & N6651 & 1981 & 382,260 & \\
\hline N6076 & 1981 & 98,803 & & N6150 & 1981 & 211,137 & & N6651 & 1982 & 491,299 & \\
\hline N6076 & 1982 & 121,112 & & N6150 & 1982 & 154,645 & & N6651 & 1983 & 372,184 & \\
\hline N6076 & 1983 & 160,772 & & N6150 & 1983 & 196,884 & & N6651 & 1984 & 214,832 & \\
\hline
\end{tabular}


Table 2B. Public-supply pumpage in Kings, Queens, and Nassau Counties, N.Y., 1898-1984--continued

\begin{tabular}{|c|c|c|c|c|c|c|c|c|c|c|c|}
\hline $\begin{array}{c}\text { Well } \\
\text { number }\end{array}$ & Year & Pumpage & Remarks & $\begin{array}{c}\text { Well } \\
\text { number }\end{array}$ & Year & Pumpage & Remarks & $\begin{array}{c}\text { Well } \\
\text { number }\end{array}$ & Year & Pumpage & Remarks \\
\hline N6744 & 1974 & 31,800 & & N6893 & 1983 & 418,875 & & N7117 & 1983 & 346,497 & \\
\hline N6744 & 1975 & 23,000 & & N6893 & 1984 & 463,594 & & N7117 & 1984 & 398,179 & \\
\hline N6744 & 1976 & 47,000 & & N6915 & 1980 & 239,982 & & N7126 & 1980 & 125,122 & \\
\hline N6744 & 1977 & 59,100 & & N6915 & 1981 & 275,818 & & N7126 & 1981 & 191,120 & \\
\hline N6744 & 1978 & 40,500 & & N6915 & 1982 & 197,011 & & N7126 & 1982 & 223,109 & \\
\hline N6744 & 1979 & 30,300 & & N6915 & 1983 & 193,816 & & N7126 & 1983 & 264,128 & \\
\hline N6744 & 1980 & 45,100 & & N6915 & 1984 & 133,097 & & N7126 & 1984 & 256,507 & \\
\hline N6744 & 1981 & 68,000 & & N6916 & 1980 & 241,955 & & N7157 & 1980 & 82,950 & \\
\hline N6744 & 1982 & 18,800 & & N6916 & 1981 & 172,194 & & N7157 & 1981 & 73,920 & \\
\hline N6744 & 1983 & 57,300 & & N6916 & 1982 & 105,335 & & N7157 & 1982 & 69,780 & \\
\hline N6744 & 1984 & 42,300 & & N6916 & 1983 & 124,912 & & N7157 & 1983 & 36,620 & \\
\hline N6745 & 1974 & 513,600 & & N6916 & 1984 & 121,424 & & N7157 & 1984 & 79,746 & \\
\hline N6745 & 1975 & 552,000 & & N6945 & 1980 & 362,300 & & N7298 & 1980 & 375,321 & \\
\hline N6745 & 1976 & 602,200 & & N6945 & 1981 & 283,289 & & N7298 & 1981 & 458,602 & \\
\hline N6745 & 1977 & 598,100 & & N6945 & 1982 & 270,268 & & N7298 & 1982 & 469,634 & \\
\hline N6745 & 1978 & 495,800 & & N6945 & 1983 & 31,812 & & N7298 & 1983 & 408,330 & \\
\hline N6745 & 1979 & 660,800 & & N6945 & 1984 & 366,924 & & N7298 & 1984 & 507,433 & \\
\hline N6745 & 1980 & 654,700 & & N6956 & 1980 & 237,661 & & N7353 & 1980 & 210,836 & \\
\hline N6745 & 1981 & 472,400 & & N6956 & 1981 & 252,478 & & N7353 & 1981 & 187,137 & \\
\hline N6745 & 1982 & 184,600 & & N6956 & 1982 & 156,049 & & N7353 & 1982 & 169,027 & \\
\hline N6745 & 1983 & 200,000 & & N6956 & 1983 & 267,437 & & N7353 & 1983 & 180,975 & \\
\hline N6745 & 1984 & 321,600 & & N6956 & 1984 & 238,771 & & N7353 & 1984 & 124,035 & \\
\hline N6817 & 1980 & 92,831 & & N7030 & 1980 & 196,558 & & N7377 & 1980 & 209,215 & \\
\hline N6817 & 1981 & 24,408 & & N7030 & 1981 & 248,027 & & N7377 & 1981 & 202,388 & \\
\hline N6817 & 1982 & 126,667 & & N7030 & 1982 & 260,303 & & N7377 & 1982 & 344,532 & \\
\hline N6817 & 1983 & 161,763 & & N7030 & 1983 & 369,401 & & N7377 & 1983 & 180,850 & \\
\hline N6817 & 1984 & 140,384 & & N7030 & 1984 & 466,549 & & N7377 & 1984 & 117,630 & \\
\hline N6819 & 1980 & 97,793 & E & N7058 & 1980 & 221,893 & & N7407 & 1980 & 179,930 & \\
\hline N6819 & 1981 & 82,124 & E & N7058 & 1981 & 237,143 & & N7407 & 1981 & 90,540 & \\
\hline N6819 & 1982 & 35,956 & E & N7058 & 1982 & 226,217 & & N7407 & 1982 & 61,110 & \\
\hline N6819 & 1983 & 30,283 & $\mathrm{E}$ & N7058 & 1983 & 287,917 & & N7407 & 1983 & 78,100 & \\
\hline N6819 & 1984 & 23,431 & $\mathrm{E}$ & N7058 & 1984 & 328,544 & & N7407 & 1984 & 88,890 & \\
\hline N6866 & 1980 & 263,898 & & N7076 & 1980 & 295,700 & & N7414 & 1980 & 213,970 & \\
\hline N6866 & 1981 & 441,486 & & N7076 & 1981 & 134,064 & & N7414 & 1981 & 188,440 & \\
\hline N6866 & 1982 & 321,465 & & N7076 & 1982 & 289,610 & & N7414 & 1982 & 207,320 & \\
\hline N6866 & 1983 & 560,664 & & N7076 & 1983 & 193,034 & & N74 14 & 1983 & 292,520 & \\
\hline N6866 & 1984 & 192,071 & & N7076 & 1984 & 263,990 & & $\mathrm{~N} 7414$ & 1984 & 458,870 & \\
\hline N6867 & 1980 & 584,788 & & N7104 & 1980 & 407,365 & & N7421 & 1980 & 287,509 & \\
\hline N6867 & 1981 & 313,974 & & N7104 & 1981 & 522,425 & & N7421 & 1981 & $319 ; 101$ & \\
\hline N6867 & 1982 & 337,037 & & N7104 & 1982 & 327,546 & & N7421 & 1982 & 277,113 & \\
\hline N6867 & 1983 & 746,085 & & N7104 & 1983 & 379,834 & & N7421 & 1983 & 226,647 & \\
\hline N6867 & 1984 & 536,470 & & N7104 & 1984 & 174,157 & & N7421 & 1984 & 199,757 & \\
\hline N6893 & 1980 & 490,606 & & N7117 & 1980 & 389,005 & & N7445 & 1974 & 2,300 & \\
\hline N6893 & 1981 & 439,019 & & N7117 & 1981 & 293,174 & & N7445 & 1975 & 390,600 & \\
\hline N6893 & 1982 & 341,088 & & N7117 & 1982 & 299,341 & & N7445 & 1976 & 314,800 & \\
\hline
\end{tabular}


Table 2B. Public-supply pumpage in Kings, Queens, and Nassau Counties, N.Y., 1898-1984--continued

\begin{tabular}{|c|c|c|c|c|c|c|c|c|c|c|c|}
\hline $\begin{array}{c}\text { Well } \\
\text { number }\end{array}$ & Year & Pumpage & Remarks & $\begin{array}{c}\text { Well } \\
\text { number }\end{array}$ & Year & Pumpage & Remarks & $\begin{array}{c}\text { Well } \\
\text { number }\end{array}$ & Year & Pumpage & Remarks \\
\hline N7445 & 1977 & 111,200 & & N7521 & 1981 & 164,512 & & N7562 & 1981 & 346,935 & \\
\hline N7445 & 1978 & 9,100 & & N7521 & 1982 & 119,202 & & N7562 & 1982 & 198,798 & \\
\hline N7445 & 1979 & 7,800 & & N7521 & 1983 & 95,934 & & N7562 & 1983 & 152,587 & \\
\hline N7445 & 1980 & 97,700 & & N7521 & 1984 & 38,220 & & N7562 & 1984 & 39,352 & \\
\hline N7445 & 1981 & 102,200 & & N7522 & 1980 & 460,152 & & N7593 & 1980 & 496,476 & \\
\hline N7445 & 1982 & 27,400 & & N7522 & 1981 & 445,803 & & N7593 & 1981 & 442,613 & \\
\hline N7445 & 1983 & 121,500 & & N7522 & 1982 & 479,917 & & N7593 & 1982 & 417,336 & \\
\hline N7445 & 1984 & 96,100 & & N7522 & 1983 & 486,760 & & N7593 & 1983 & 221,798 & \\
\hline N7446 & 1980 & 131,892 & & N7522 & 1984 & 494,041 & & N7593 & 1984 & 378,102 & \\
\hline N7446 & 1981 & 135,888 & & N7523 & 1980 & 232,498 & & N7620 & 1980 & 21,430 & \\
\hline N7446 & 1982 & 155,759 & & N7523 & 1981 & 159,565 & & N7620 & 1981 & 22,133 & \\
\hline N7446 & 1983 & 134,624 & & N7523 & 1982 & 211,117 & & N7620 & 1982 & 29,569 & \\
\hline N7446 & 1984 & 163,937 & & N7523 & 1983 & 220,973 & & N7620 & 1983 & 32,731 & \\
\hline N7482 & 1974 & 469,400 & & N7523 & 1984 & 166,881 & & N7620 & 1984 & 35,644 & \\
\hline N7482 & 1975 & 608,600 & & N7526 & 1980 & 100,393 & & N7643 & 1980 & 435 & \\
\hline N7482 & 1976 & 591,900 & & N7526 & 1981 & 66,314 & & N7643 & 1981 & 340 & \\
\hline N7482 & 1977 & 533,400 & & N7526 & 1982 & 74,268 & & N7643 & 1982 & 1,656 & \\
\hline N7482 & 1978 & 408,800 & & N7526 & 1983 & 87,169 & & N7643 & 1983 & 4,013 & \\
\hline N7482 & 1979 & 535,700 & & N7526 & 1984 & 72,491 & & N7649 & 1974 & 109,200 & \\
\hline N7482 & 1980 & 617,500 & & N7548 & 1980 & 582,061 & & N7649 & 1975 & 41,100 & \\
\hline N7482 & 1981 & 252,400 & & N7548 & 1981 & 576,956 & & N7649 & 1976 & 170,400 & \\
\hline N7482 & 1982 & 262,700 & & N7548 & 1982 & 573,829 & & N7649 & 1977 & 170,500 & \\
\hline N7482 & 1983 & 559,400 & & N7548 & 1983 & 504,554 & & N7649 & 1978 & 301,300 & \\
\hline N7482 & 1984 & 726,700 & & N7548 & 1984 & 539,204 & & N7649 & 1979 & 14,100 & \\
\hline N7512 & 1980 & 242,659 & & N7549 & 1980 & 169,887 & & N7649 & 1980 & 228,300 & \\
\hline N7512 & 1981 & 299,227 & & N7549 & 1981 & 148,857 & & N7649 & 1981 & 274,500 & \\
\hline N7512 & 1982 & 435,814 & & N7549 & 1982 & 156,193 & & N7649 & 1982 & 196,400 & \\
\hline N7512 & 1983 & 324,259 & & N7549 & 1983 & 184,982 & & N7649 & 1983 & 34,600 & \\
\hline N7512 & 1984 & 432,828 & & N7549 & 1984 & 134,406 & & N7649 & 1984 & 100 & \\
\hline N7513 & 1980 & 139,410 & & N7551 & 1980 & 204,560 & & N7650 & 1974 & 419,300 & \\
\hline N7513 & 1981 & 153,413 & & N7551 & 1981 & 221,040 & & N7650 & 1975 & 407,300 & \\
\hline N7513 & 1982 & 128,133 & & N7551 & 1982 & 414,410 & & N7650 & 1976 & 505,800 & \\
\hline N7513 & 1983 & 157,106 & & N755l & 1983 & 473,050 & & N7650 & 1977 & 449,600 & \\
\hline N7513 & 1984 & 173,888 & & N7551 & 1984 & 437,860 & & N7650 & 1978 & 541,000 & \\
\hline N7515 & 1980 & 200,347 & & N7552 & 1980 & 421,280 & & N7650 & 1979 & 416,000 & \\
\hline N7515 & 1981 & 121,884 & & N7552 & 1981 & 443,710 & & N7650 & 1980 & 527,700 & \\
\hline N7515 & 1982 & 272,816 & & N7552 & 1982 & 425,900 & & N7650 & 1981 & 552,700 & \\
\hline N7515 & 1983 & 302,979 & & N7552 & 1983 & 427,380 & & N7650 & 1982 & 427,000 & \\
\hline N7515 & 1984 & 251,646 & & N7552 & 1984 & 366,910 & & N7650 & 1983 & 508,800 & \\
\hline N7516 & 1980 & 71,650 & & N7561 & 1980 & 336,758 & & N7650 & 1984 & 316,100 & \\
\hline N7516 & 1981 & 127,813 & & N7561 & 1981 & 126,432 & & N7651 & 1980 & 413,509 & \\
\hline N7516 & 1982 & 71,866 & & N7561 & 1982 & 138,298 & & N7651 & 1981 & 483,808 & \\
\hline N7516 & 1983 & 65,054 & & N7561 & 1983 & 114,008 & & N7651 & 1982 & 510,588 & \\
\hline N7516 & 1984 & 179,819 & & N7561 & 1984 & 65,860 & & N7651 & 1983 & 453,749 & \\
\hline N7521 & 1980 & 91,705 & & N7562 & 1980 & 514,164 & & N7561 & 1984 & 360,189 & \\
\hline
\end{tabular}


Table 2B. Public-supply pumpage in Kings, Queens, and Nassau Counties, N.Y., 1898-1984--continued

\begin{tabular}{|c|c|c|c|c|c|c|c|c|c|c|c|}
\hline $\begin{array}{c}\text { Well } \\
\text { number }\end{array}$ & Year & Pumpage & Remarks & $\begin{array}{c}\text { Well } \\
\text { number }\end{array}$ & Year & Pumpage & Remarks & $\begin{array}{c}\text { Well } \\
\text { number }\end{array}$ & Year & Pumpage & Remarks \\
\hline N7665 & 1980 & 198,635 & & N7755 & 1984 & 3,686 & $\mathbf{E}$ & N7797 & 1982 & 267,779 & \\
\hline N7665 & 1981 & 341,494 & & N7756 & 1981 & 372 & $\mathbf{E}$ & N7797 & 1983 & 225,832 & \\
\hline N7665 & 1982 & 239,111 & & N7756 & 1982 & 251 & $\mathrm{E}$ & N7797 & 1984 & 106,217 & \\
\hline N7665 & 1983 & 70,788 & & N7756 & 1983 & 483 & $\mathbf{E}$ & N7831 & 1980 & 718,212 & \\
\hline N7665 & 1984 & 341,586 & & N7756 & 1984 & 3,686 & E & N7831 & 1981 & 614,407 & \\
\hline N7720 & 1980 & 107,891 & & N7757 & 1981 & 372 & $\mathrm{E}$ & N7831 & 1982 & 737,080 & \\
\hline N7720 & 1981 & 48,883 & & N7757 & 1982 & 251 & E & N7831 & 1983 & 702,894 & \\
\hline N7720 & 1982 & 66,306 & & N7757 & 1983 & 483 & E & N7831 & 1984 & 591,771 & \\
\hline N7720 & 1983 & 89,200 & & N7757 & 1984 & 3,686 & $\mathbf{E}$ & N7852 & 1980 & 179,063 & \\
\hline N7720 & 1984 & 98,921 & & N7758 & 1981 & 372 & E & N7852 & 1981 & 178,057 & \\
\hline N7747 & 1981 & 372 & $\mathrm{E}$ & N7758 & 1982 & 251 & $\mathbf{E}$ & N7852 & 1982 & 201,538 & \\
\hline N7747 & 1982 & 251 & $\mathrm{E}$ & N7758 & 1983 & 483 & $\mathbf{E}$ & N7852 & 1983 & 213,747 & \\
\hline N7747 & 1983 & 483 & $\mathrm{E}$ & N7758 & 1984 & 3,686 & $\mathrm{E}$ & N7852 & 1984 & 203,428 & \\
\hline N7747 & 1984 & 3,686 & $\mathrm{E}$ & N7772 & 1980 & 202,367 & & N7855 & 1980 & 485,316 & \\
\hline N7748 & 1981 & 372 & $\mathrm{E}$ & N7772 & 1981 & 132,544 & & N7855 & 1981 & 378,475 & \\
\hline N7748 & 1982 & 251 & $\mathrm{E}$ & N7772 & 1982 & 96,895 & & N7855 & 1982 & 360,031 & \\
\hline N7748 & 1983 & 483 & $\mathbf{E}$ & N7772 & 1983 & 125,777 & & N7855 & 1983 & 471,381 & \\
\hline N7748 & 1984 & 3,686 & $\mathrm{E}$ & N7772 & 1984 & 136,261 & & N7855 & 1984 & 370,634 & \\
\hline N7749 & 1981 & 372 & $\mathrm{E}$ & N7773 & 1980 & 84,457 & & N7857 & 1980 & 79,297 & \\
\hline N7749 & 1982 & 251 & $\mathbf{E}$ & $N 7773$ & 1981 & 62,521 & & N7857 & 1981 & 53,766 & \\
\hline N7749 & 1983 & 483 & $\mathrm{E}$ & N7773 & 1982 & 47,016 & & N7857 & 1982 & 38,821 & \\
\hline N7749 & 1984 & 3,686 & $\mathrm{E}$ & N7773 & 1983 & 101,512 & & N7857 & 1983 & 62,670 & \\
\hline N7750 & 1981 & 372 & $\mathbf{E}$ & N7773 & 1984 & 68,859 & & N7857 & 1984 & 125,116 & \\
\hline N7750 & 1982 & 251 & $\mathrm{E}$ & N7776 & 1980 & 274,159 & & N7873 & 1980 & 177,507 & \\
\hline N7750 & 1983 & 483 & $\mathrm{E}$ & N7776 & 1981 & 234,529 & & N7873 & 1981 & 92,304 & \\
\hline N7750 & 1984 & 3,686 & $\mathrm{E}$ & N7776 & 1982 & 217,900 & & N7873 & 1982 & 84,961 & \\
\hline N7751 & 1981 & 372 & $\mathrm{E}$ & N7776 & 1983 & 113,407 & & N7873 & 1983 & 132,537 & \\
\hline N7751 & 1982 & 251 & $\mathrm{E}$ & N7776 & 1984 & 64,013 & & N7873 & 1984 & 77,249 & \\
\hline N7751 & 1983 & 483 & $\mathrm{E}$ & N7781 & 1980 & 243,316 & & N7892 & 1980 & 116,681 & \\
\hline N7751 & 1984 & 3,686 & $\mathrm{E}$ & N7781 & 1981 & 225,897 & & N7892 & 1981 & 122,097 & \\
\hline N7752 & 1981 & 372 & $\mathrm{E}$ & N7781 & 1982 & 258,617 & & N7892 & 1982 & 168,422 & \\
\hline N7752 & 1982 & 251 & E & N7781 & 1983 & 229,641 & & N7892 & 1983 & 59,889 & \\
\hline N7752 & 1983 & 483 & $\mathrm{E}$ & N7781 & 1984 & 157,414 & & N7892 & 1984 & 19,051 & \\
\hline N7752 & 1984 & 3,686 & $\mathrm{E}$ & N7785 & 1980 & 154,637 & & N7957 & 1980 & 403,129 & \\
\hline N7753 & 1981 & 372 & $\mathrm{E}$ & N7785 & 1981 & 144,701 & & N7957 & 1981 & 482,459 & \\
\hline N7753 & 1982 & 251 & $\mathrm{E}$ & N7785 & 1982 & 132,190 & & N7957 & 1982 & 199,025 & \\
\hline N7753 & 1983 & 483 & $\mathrm{E}$ & N7785 & 1983 & 117,060 & & N7957 & 1983 & 262,335 & \\
\hline N7753 & 1984 & 3,686 & $\mathrm{E}$ & N7785 & 1984 & 124,048 & & N7957 & 1984 & 227,974 & \\
\hline N7754 & 1981 & 372 & $\mathrm{E}$ & N7796 & 1980 & 218,166 & & N7961 & 1981 & 372 & $\mathrm{E}$ \\
\hline N7754 & 1982 & 251 & $\mathbf{E}$ & N7796 & 1981 & 242,116 & & N7961 & 1982 & 251 & $\mathrm{E}$ \\
\hline N7754 & 1983 & 483 & $E$ & N7796 & 1982 & 184,527 & & N7961 & 1983 & 483 & $\mathrm{E}$ \\
\hline N7754 & 1984 & 3,686 & $\mathrm{E}$ & N7796 & 1983 & 271,317 & & N7961 & 1984 & 3,686 & $\mathrm{E}$ \\
\hline N7755 & 1981 & 372 & E & N7796 & 1984 & 239,379 & & N7962 & 1981 & 372 & $\mathbf{E}$ \\
\hline N7755 & 1982 & 251 & E & N7797 & 1980 & 237,213 & & N7962 & 1982 & 251 & $\mathrm{E}$ \\
\hline N7755 & 1983 & 483 & $\mathrm{E}$ & N7797 & 1981 & 421,818 & & N7962 & 1983 & 483 & $\mathrm{E}$ \\
\hline
\end{tabular}


Table 2B. Public-supply pumpage in Kings, Queens, and Nassau Counties, N.Y., 1898-1984--continued

\begin{tabular}{|c|c|c|c|c|c|c|c|c|c|c|c|}
\hline $\begin{array}{c}\text { Well } \\
\text { number }\end{array}$ & Year & Pumpage & Remarks & $\begin{array}{c}\text { Well } \\
\text { number }\end{array}$ & Year & Pumpage & Remarks & $\begin{array}{c}\text { Well } \\
\text { number }\end{array}$ & Year & Pumpage & Remarks \\
\hline N7962 & 1984 & 3,686 & $\mathrm{E}$ & N8031 & 1984 & 793,240 & & N8233 & 1981 & 27,360 & \\
\hline N7963 & 1981 & 372 & $\mathrm{E}$ & N8043 & 1980 & 338,461 & & N8233 & 1982 & 83,100 & \\
\hline N7963 & 1982 & 251 & $\mathrm{E}$ & N8043 & 1981 & 225,520 & & N8233 & 1983 & 237,473 & \\
\hline N7963 & 1983 & 483 & $\mathrm{E}$ & N8043 & 1982 & 207,812 & & N8233 & 1984 & 154,797 & \\
\hline N7963 & 1984 & 3,686 & $\mathrm{E}$ & N8043 & 1983 & 480,059 & & N8248 & 1980 & 319,330 & \\
\hline N7964 & 1981 & 372 & E & N8043 & 1984 & 202,452 & & N8248 & 1981 & 313,460 & \\
\hline N7964 & 1982 & 251 & $\mathbf{E}$ & N8054 & 1980 & 256,557 & & N8248 & 1982 & 234,290 & \\
\hline N7964 & 1983 & 483 & E & N8054 & 1981 & 244,633 & & N8248 & 1983 & 204,690 & \\
\hline N7964 & 1984 & 3,686 & E & N8054 & 1982 & 314,675 & & N8248 & 1984 & 167,660 & \\
\hline N7965 & 1981 & 372 & $\mathbf{E}$ & N8054 & 1983 & 251,129 & & N8249 & 1980 & 46,035 & \\
\hline N7965 & 1982 & 251 & $\mathrm{E}$ & N8054 & 1984 & 236,733 & & N8249 & 1981 & 67,294 & \\
\hline N7965 & 1983 & 483 & $\mathrm{E}$ & N8183 & 1980 & 91,763 & & N8249 & 1982 & 4,134 & \\
\hline N7965 & 1984 & 3,686 & $\mathrm{E}$ & N8183 & 1981 & 31,775 & & N8250 & 1980 & 43,878 & \\
\hline N7966 & 1981 & 372 & $\mathrm{E}$ & N8183 & 1982 & 29,171 & & N8250 & 1981 & 20,647 & \\
\hline N7966 & 1982 & 251 & $\mathrm{E}$ & N8183 & 1983 & 25,300 & & N8250 & 1982 & 21,293 & \\
\hline N7966 & 1983 & 483 & E & N8183 & 1984 & 25,488 & & N8250 & 1983 & 44,697 & \\
\hline N7966 & 1984 & 3,686 & E & N8195 & 1980 & 466,675 & & $\mathrm{~N} 8250$ & 1984 & 24,221 & \\
\hline N7968 & 1981 & 372 & E & N8195 & 1981 & 538,423 & & N8251 & 1980 & 497,211 & \\
\hline N7968 & 1982 & 251 & E & N8195 & 1982 & 479,628 & & N8251 & 1981 & 151,106 & \\
\hline N7968 & 1983 & 483 & $\mathrm{E}$ & N8195 & 1983 & 555,282 & & N8251 & 1982 & 186,976 & \\
\hline N7968 & 1984 & 3,686 & $\mathrm{E}$ & N8195 & 1984 & 502,206 & & N8251 & 1983 & 389,004 & \\
\hline N8004 & 1980 & 79,835 & & N8196 & 1980 & 479,090 & & N8251 & 1984 & 401,644 & \\
\hline N8004 & 1981 & 50,960 & & N8196 & 1981 & 439,951 & & N8253 & 1980 & 20,290 & \\
\hline N8004 & 1982 & 45,445 & & N8196 & 1982 & 433,289 & & N8253 & 1981 & 61,080 & \\
\hline N8004 & 1983 & 204,105 & & N8196 & 1983 & 488,109 & & N8253 & 1982 & 84,430 & \\
\hline N8004 & 1984 & 76,955 & & N8196 & 1984 & 531,557 & & N8253 & 1983 & 148,140 & \\
\hline N8007 & 1980 & 98,360 & & N8214 & 1980 & 398,795 & & N8253 & 1984 & 64,860 & \\
\hline N8007 & 1981 & 72,853 & & N8214 & 1981 & 245,583 & & N8264 & 1980 & 443,445 & \\
\hline N8007 & 1982 & 90,718 & & N8214 & 1982 & 308,827 & & N8264 & 1981 & 210,358 & \\
\hline N8007 & 1983 & 120,772 & & N8214 & 1983 & 82,067 & & N8264 & 1982 & 117,583 & \\
\hline N8007 & 1984 & 142,120 & & N8214 & 1984 & 231,682 & & N8264 & 1983 & 48,525 & \\
\hline N8010 & 1980 & 28,755 & & N8216 & 1980 & 253,283 & & N8264 & 1984 & 86,265 & \\
\hline N8010 & 1981 & 72,351 & & N8216 & 1981 & 265,823 & & N8279 & 1980 & 214,719 & \\
\hline N8010 & 1982 & 98,476 & & N8216 & 1982 & 136,634 & & N8279 & 1981 & 80,948 & \\
\hline N8010 & 1983 & 172,654 & & N8216 & 1983 & 289,003 & & N8279 & 1982 & 139,958 & \\
\hline N8010 & 1984 & 42,508 & & N8216 & 1984 & 282,459 & & N8279 & 1983 & 214,130 & \\
\hline N8011 & 1980 & 157,684 & & N8217 & 1980 & 221,015 & & N8279 & 1984 & 188,884 & \\
\hline N8011 & 1981 & 10,740 & & N8217 & 1981 & 194,664 & & N8313 & 1980 & 91,720 & \\
\hline N8011 & 1982 & 175,960 & & N8217 & 1982 & 90,753 & & N8313 & 1981 & 92,690 & \\
\hline N8011 & 1983 & 94,403 & & N8217 & 1983 & 21,641 & & N8313 & 1982 & 97,530 & \\
\hline N8011 & 1984 & 284,527 & & N8218 & 1980 & 183,105 & & N8313 & 1983 & 134,240 & \\
\hline N8031 & 1980 & 533,350 & & N8218 & 1981 & 128,268 & & N8313 & 1984 & 95,790 & \\
\hline N8031 & 1981 & 729,650 & & N8218 & 1982 & 171,615 & & N8321 & 1980 & 197,020 & \\
\hline N8031 & 1982 & 679,380 & & N8218 & 1983 & 158,762 & & N8321 & 1981 & 138,839 & \\
\hline N8031 & 1983 & 721,370 & & N8218 & 1984 & 127,994 & & N8321 & 1982 & 190,264 & \\
\hline
\end{tabular}


Table 2B. Public-supply pumpage in Kings, Queens, and Nassau Counties, N.Y., 1898-1984--continued

\begin{tabular}{|c|c|c|c|c|c|c|c|c|c|c|c|}
\hline $\begin{array}{c}\text { Well } \\
\text { number }\end{array}$ & Year & Pumpage & Remarks & $\begin{array}{c}\text { Well } \\
\text { number }\end{array}$ & Year & Pumpage & Remarks & $\begin{array}{c}\text { Well } \\
\text { number }\end{array}$ & Year & Pumpage & Remarks \\
\hline N8321 & 1983 & 147,780 & & N8475 & 1983 & 159,532 & & N8603 & 1983 & 762,970 & \\
\hline N8321 & 1984 & 138,430 & & N8475 & 1984 & 248,565 & & N8603 & 1984 & 667,600 & \\
\hline N8326 & 1983 & 405 & & N8480 & 1980 & 729,720 & & N8657 & 1980 & 261,051 & \\
\hline N8339 & 1980 & 260,625 & & N8480 & 1981 & 798,010 & & N8657 & 1981 & 229,891 & \\
\hline N8339 & 1981 & 223,603 & & N8480 & 1982 & 627,140 & & N8657 & 1982 & 161,279 & \\
\hline N8339 & 1982 & 198,779 & & N8480 & 1983 & 345,430 & & N8657 & 1983 & 244,447 & \\
\hline N8339 & 1983 & 257,386 & & N8480 & 1984 & 74,230 & & N8657 & 1984 & 344,324 & \\
\hline N8339 & 1984 & 309,050 & & N8497 & 1980 & 219,771 & & N8658 & 1980 & 159,968 & \\
\hline N8342 & 1980 & 10,970 & & N8497 & 1981 & 273,431 & & N8658 & 1981 & 132,492 & \\
\hline N8342 & 1981 & 13,710 & & N8497 & 1982 & 261,642 & & N8658 & 1982 & 149,786 & \\
\hline N8342 & 1982 & 23,763 & & N8497 & 1983 & 291,085 & & N8658 & 1983 & 178,728 & \\
\hline N8342 & 1983 & 136,631 & & N8497 & 1984 & 229,838 & & N8658 & 1984 & 136,312 & \\
\hline N8342 & 1984 & 242,047 & & N8525 & 1980 & 52,969 & & N8664 & 1980 & 324,976 & \\
\hline N8354 & 1980 & 162,844 & & N8525 & 1981 & 21,992 & & N8664 & 1981 & 477,436 & \\
\hline N8354 & 1981 & 153,228 & & N8525 & 1982 & 3,635 & & N8664 & 1982 & 373,130 & \\
\hline N8354 & 1982 & 168,548 & & N8525 & 1983 & 26,522 & & N8664 & 1983 & 143,192 & \\
\hline N8354 & 1983 & 159,494 & & N8525 & 1984 & 30,036 & & N8664 & 1984 & 195,019 & \\
\hline N8354 & 1984 & 156,337 & & N8526 & 1980 & 107,285 & & N8665 & 1980 & 118,595 & \\
\hline N8355 & 1980 & 91,285 & & N8526 & 1981 & 136,047 & & N8665 & 1981 & 8,745 & \\
\hline N8355 & 1981 & 35,874 & & N8526 & 1982 & 47,801 & & N8665 & 1982 & 17,278 & \\
\hline N8355 & 1982 & 55,359 & & N8526 & 1983 & 74,285 & & N8665 & 1983 & 209,070 & \\
\hline N8355 & 1983 & 49,951 & & N8526 & 1984 & 15,699 & & N8665 & 1984 & 4,326 & \\
\hline N8355 & 1984 & 72,553 & & N8557 & 1980 & 133,725 & & N8713 & 1980 & 129,374 & \\
\hline N8409 & 1981 & 17,195 & & N8557 & 1981 & 208,638 & & N8713 & 1981 & 116,936 & \\
\hline N8409 & 1982 & 26,179 & & N8557 & 1982 & 381,304 & & N8713 & 1982 & 114,266 & \\
\hline N8409 & 1983 & 79,709 & & N8557 & 1983 & 72,990 & & N8713 & 1983 & 76,027 & \\
\hline N8409 & 1984 & 20,758 & & N8557 & 1984 & 123,940 & & N8713 & 1984 & 184,603 & \\
\hline N8420 & 1980 & 147,097 & & N8558 & 1980 & 240,052 & & N8767 & 1980 & 394,408 & \\
\hline N8420 & 1981 & 87,325 & & N8558 & 1981 & 295,275 & & N8767 & 1981 & 462,023 & \\
\hline N8420 & 1982 & 83,964 & & N8558 & 1982 & 202,006 & & N8767 & 1982 & 658,436 & \\
\hline N8420 & 1983 & 82,163 & & N8558 & 1983 & 297,566 & & N8767 & 1983 & 284,831 & \\
\hline N8420 & 1984 & 40,068 & & N8558 & 1984 & 227,272 & & N8767 & 1984 & 372,152 & \\
\hline N8457 & 1980 & 172,251 & & N8576 & 1980 & 460,805 & & N8768 & 1980 & 372,438 & \\
\hline N8457 & 1981 & 175,025 & & N8576 & 1981 & 477,674 & & N8768 & 1981 & 275,030 & \\
\hline N8457 & 1982 & 147,296 & & N8576 & 1982 & 395,305 & & N8768 & 1982 & 246,556 & \\
\hline N8457 & 1983 & 162,481 & & N8576 & 1983 & 337,727 & & N8768 & 1983 & 131,124 & \\
\hline N8457 & 1984 & 179,226 & & N8576 & 1984 & 341,677 & & N8768 & 1984 & 127,025 & \\
\hline N8474 & 1980 & 198,610 & & N8595 & 1980 & 100,369 & & N8776 & 1980 & 274,445 & \\
\hline N8474 & 1981 & 207,767 & & N8595 & 1981 & 156,769 & & N8776 & 1981 & 292,586 & \\
\hline N8474 & 1982 & 273,607 & & N8595 & 1982 & 169,395 & & N8776 & 1982 & 296,564 & \\
\hline N8474 & 1983 & 183,416 & & N8595 & 1983 & 218,638 & & N8776 & 1983 & 318,986 & \\
\hline N8474 & 1984 & 218,243 & & N8595 & 1984 & 136,551 & & N8776 & 1984 & 315,615 & \\
\hline N8475 & 1980 & 290,698 & & N8603 & 1980 & 849,920 & & N8778 & 1980 & 441,230 & \\
\hline N8475 & 1981 & 225,774 & & N8603 & 1981 & 778,160 & & N8778 & 1981 & 228,447 & \\
\hline N8475 & 1982 & 299,475 & & N8603 & 1982 & 811,960 & & N8778 & 1982 & 214,866 & \\
\hline
\end{tabular}


Table 2B. Public-supply pumpage in Kings, Queens, and Nassau Counties, N.Y., 1898-1984--continued

\begin{tabular}{|c|c|c|c|c|c|c|c|c|c|c|c|}
\hline $\begin{array}{c}\text { Well } \\
\text { number }\end{array}$ & Year & Pumpage & Remarks & $\begin{array}{c}\text { Well } \\
\text { number }\end{array}$ & Year & Pumpage & Remarks & $\begin{array}{c}\text { Well } \\
\text { number }\end{array}$ & Year & Pumpage & Remarks \\
\hline N8778 & 1983 & 154,499 & & N9151 & 1980 & 330,000 & $E$ & N9452 & 1983 & 27,718 & \\
\hline N8778 & 1984 & 233,541 & & N9151 & 1981 & 245,300 & $\mathrm{E}$ & N9452 & 1984 & 56,804 & \\
\hline N8779 & 1980 & 149,492 & & N915I & 1982 & 288,300 & $\mathrm{E}$ & N9463 & 1981 & 447,744 & \\
\hline N8779 & 1981 & 114,617 & & N9151 & 1983 & 285,850 & $\mathrm{E}$ & N9463 & 1982 & 675,911 & \\
\hline N8779 & 1982 & 169,614 & & N9151 & 1984 & 240,450 & $\mathrm{E}$ & N9463 & 1983 & 610,393 & \\
\hline N8779 & 1983 & 172,555 & & N9173 & 1980 & 542,251 & & N9463 & 1984 & 526,920 & \\
\hline N8779 & 1984 & 156,238 & & N9173 & 1981 & 172,140 & & N9488 & 1981 & 165,112 & \\
\hline N8818 & 1980 & 179,057 & & N9173 & 1982 & 521,867 & & N9488 & 1982 & 274,534 & \\
\hline N8818 & 1981 & 255,376 & & N9173 & 1983 & 186,556 & & N9488 & 1983 & 452,388 & \\
\hline N8818 & 1982 & 245,193 & & N9173 & 1984 & 94,878 & & N9488 & 1984 & 624,775 & \\
\hline N8818 & 1983 & 243,593 & & N9180 & 1980 & 408,397 & & N9514 & 1980 & 275,380 & \\
\hline N8818 & 1984 & 217,349 & & N9180 & 1981 & 398,093 & & N9514 & 1981 & 602,580 & \\
\hline N8837 & 1980 & 391,030 & & N9180 & 1982 & 276,669 & & N9514 & 1982 & 913,570 & \\
\hline N8837 & 1981 & 143,100 & & N9180 & 1983 & 437,751 & & N9514 & 1983 & 859,340 & \\
\hline N8837 & 1982 & 80,390 & & N9180 & 1984 & 573,888 & & N9514 & 1984 & 453,360 & \\
\hline N8837 & 1983 & 44,750 & & N9210 & 1980 & 371,065 & & N9520 & 1981 & 72,517 & \\
\hline N8837 & 1984 & 32,060 & & N9210 & 1981 & 262,314 & & N9520 & 1982 & 99,400 & \\
\hline N8941 & 1980 & 121,747 & & N9210 & 1982 & 286,719 & & N9520 & 1983 & 115,375 & \\
\hline N8941 & 1981 & 95,188 & & N9210 & 1983 & 265,219 & & N9520 & 1984 & 88,462 & \\
\hline N8941 & 1983 & 212,294 & & N9210 & 1984 & 267,907 & & N9521 & 1981 & 191,868 & \\
\hline N8941 & 1984 & 403,151 & & N9211 & 1980 & 373,754 & & N9521 & 1982 & 308,595 & \\
\hline N8956 & 1980 & 162,695 & & N9211 & 1981 & 344,252 & & N9521 & 1983 & 224,388 & \\
\hline N8956 & 1981 & 134,325 & & N9211 & 1982 & 290,073 & & N9521 & 1984 & 191,570 & \\
\hline N8956 & 1982 & 261,947 & & N9211 & 1983 & 269,881 & & N9591 & 1983 & 406,771 & \\
\hline N8956 & 1983 & 379,231 & & N9211 & 1984 & 271,473 & & N9591 & 1984 & 100,898 & \\
\hline N8956 & 1984 & 208,319 & & N9212 & 1980 & 251,105 & & N9613 & 1981 & 95,482 & \\
\hline N8957 & 1980 & 388,433 & & N9212 & 1981 & 365,726 & & N9613 & 1982 & 145,214 & \\
\hline N8957 & 1981 & 126,671 & & N9212 & 1982 & 427,038 & & N9613 & 1983 & 161,494 & \\
\hline N8957 & 1982 & 305,943 & & N921.2 & 1983 & 402,920 & & N9613 & 1984 & 94,002 & \\
\hline N8957 & 1983 & 266,950 & & N9212 & 1984 & 323,296 & & N9768 & 1982 & 281,980 & \\
\hline N8957 & 1984 & 302,077 & & N9308 & 1981 & 68,675 & & N9768 & 1983 & 386,874 & \\
\hline N8976 & 1980 & 439,970 & & N9308 & 1982 & 15,643 & & N9768 & 1984 & 319,055 & \\
\hline N8976 & 1981 & 477,710 & & N9308 & 1983 & 99,359 & & N9792 & 1982 & 134,116 & \\
\hline N8976 & 1982 & 689,110 & & N9308 & 1984 & 45,964 & & N9792 & 1983 & 170,542 & \\
\hline N8976 & 1983 & 362,720 & & N9334 & 1980 & 280,757 & & N9792 & 1984 & 171,026 & \\
\hline N8976 & 1984 & 323,170 & & N9334 & 1981 & 570,594 & & N9809 & 1984 & 154,970 & \\
\hline N8979 & 1980 & 91,703 & & N9334 & 1982 & 511,041 & & N9846 & 1983 & 153,548 & \\
\hline N8979 & 1981 & 27,037 & & N9334 & 1983 & 622,480 & & N9846 & 1984 & 366,438 & \\
\hline N8979 & 1982 & 38,999 & & N9334 & 1984 & 682,467 & & N9878 & 1982 & 192,490 & \\
\hline N8979 & 1983 & 86,018 & & N9338 & 1980 & 259,440 & & N9878 & 1983 & 493,280 & \\
\hline N8979 & 1984 & 41,999 & & N9338 & 1981 & 125,340 & & N9878 & 1984 & 565,000 & \\
\hline N9151 & 1974 & 403,550 & $\mathrm{E}$ & N9338 & 1982 & 325,230 & & N9910 & 1983 & 428,360 & \\
\hline N9151 & 1975 & 320,850 & $\mathrm{E}$ & N9338 & 1983 & 690,920 & & N9910 & 1984 & 553,920 & \\
\hline N9151 & 1976 & 257,600 & E & N9338 & 1984 & $1,000,990$ & & N9976 & 1983 & 129,950 & \\
\hline N9151 & 1977 & 224,700 & $\mathrm{E}$ & N9446 & 1983 & 6,418 & & N9976 & 1984 & 203,870 & \\
\hline N9151 & 1978 & 373,400 & $\mathrm{E}$ & N9452 & 1981 & 25,781 & & N10033 & 1984 & 37,310 & \\
\hline N9151 & 1979 & 338,300 & $\mathrm{E}$ & N9452 & 1982 & 50,684 & & N10144 & 1984 & 2,201 & \\
\hline
\end{tabular}




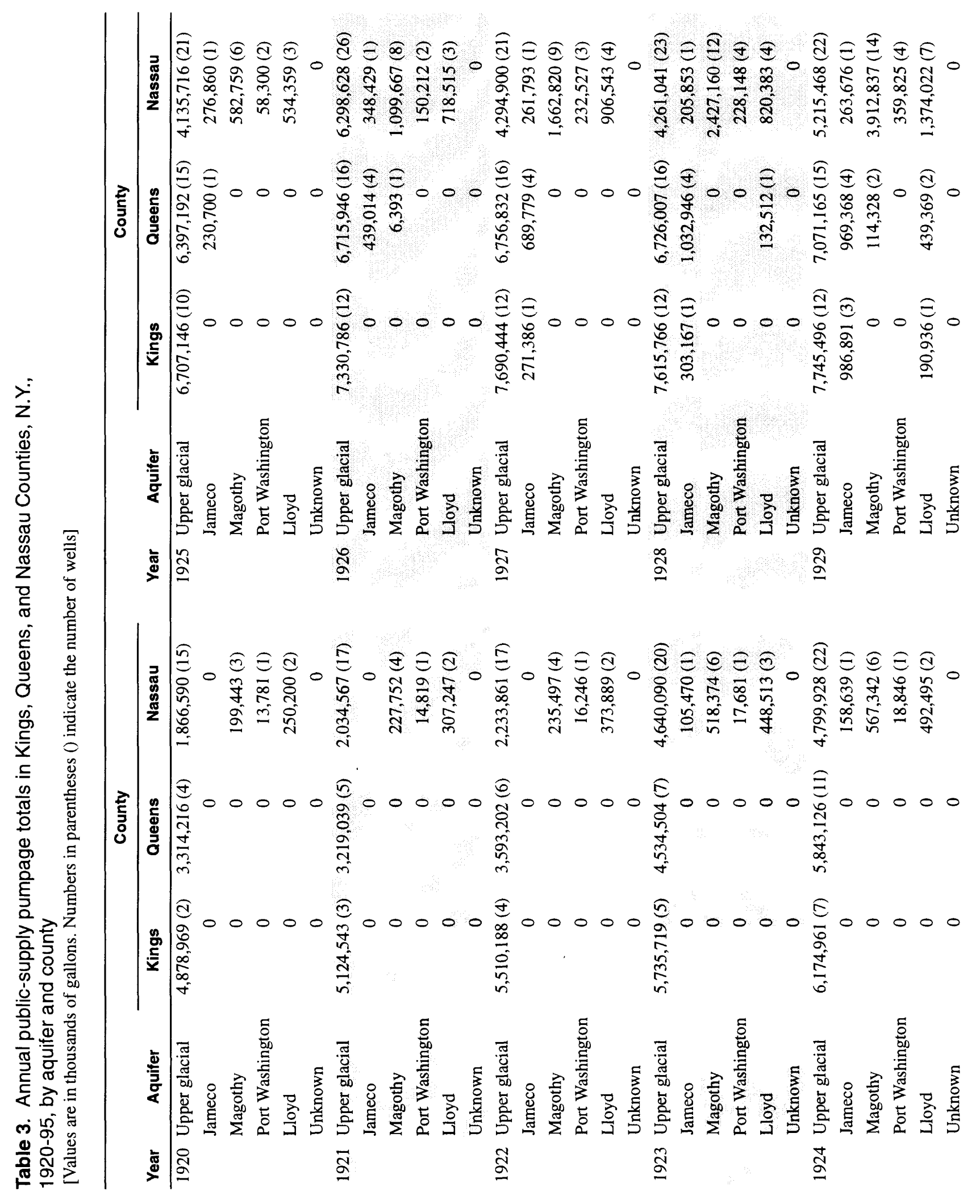




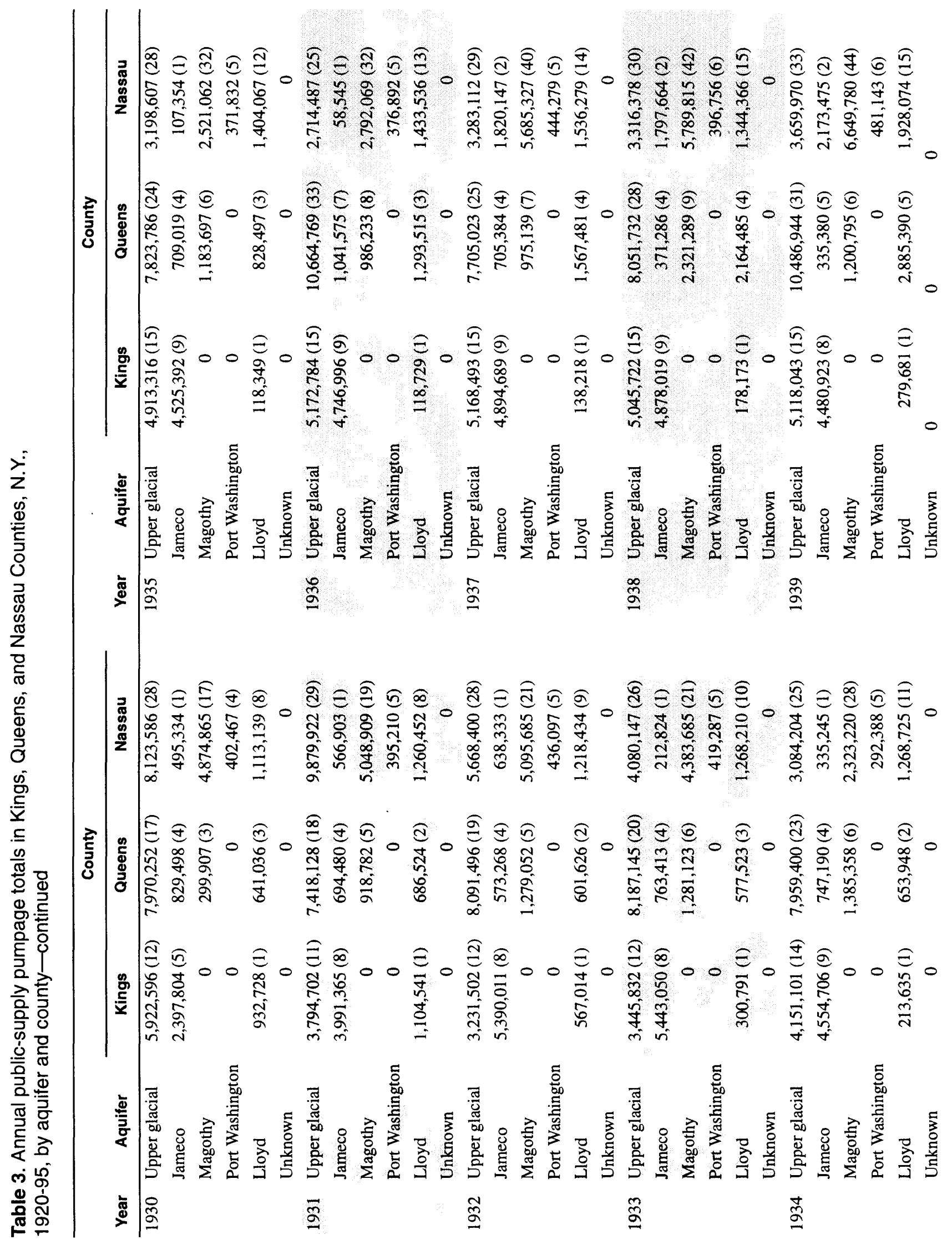




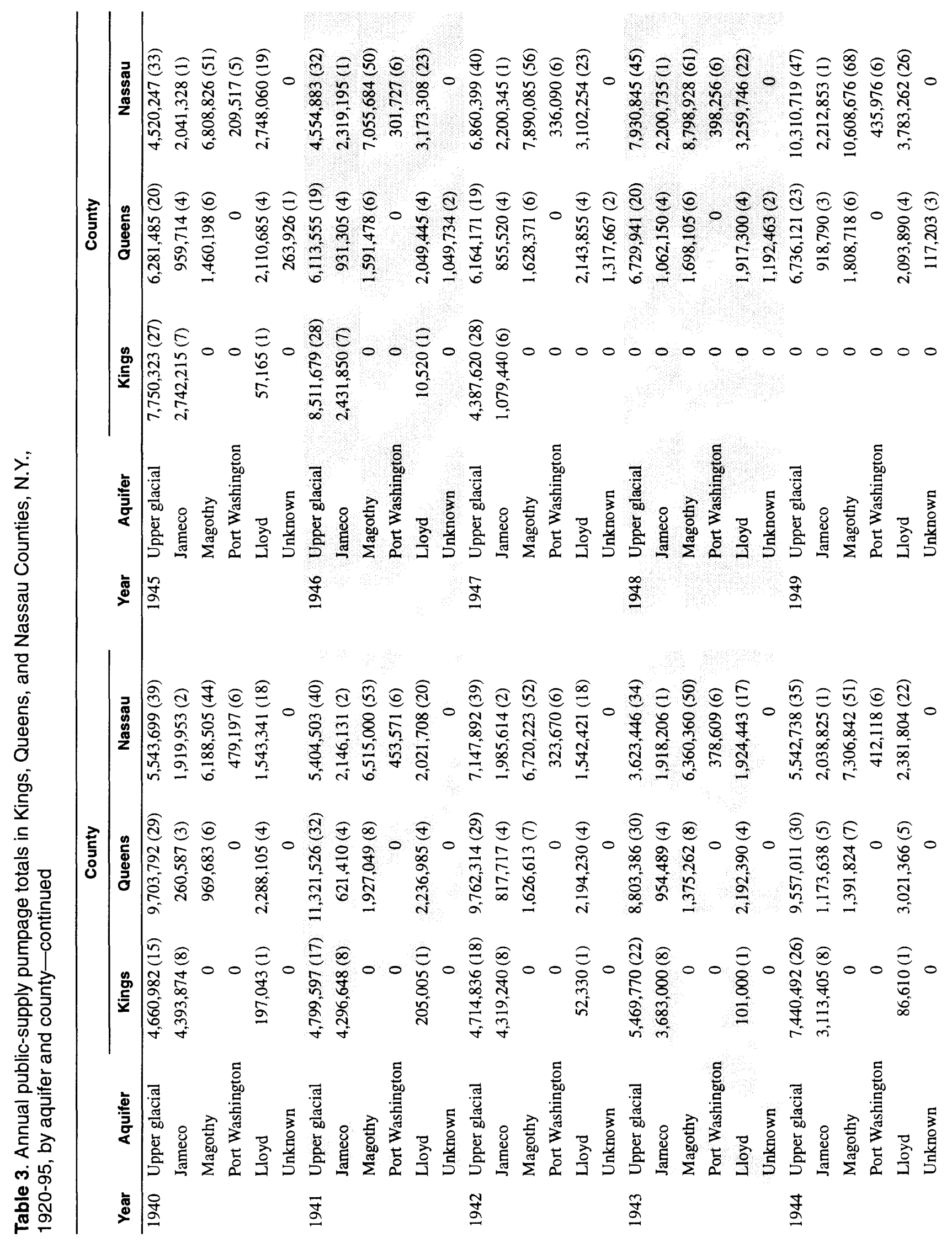




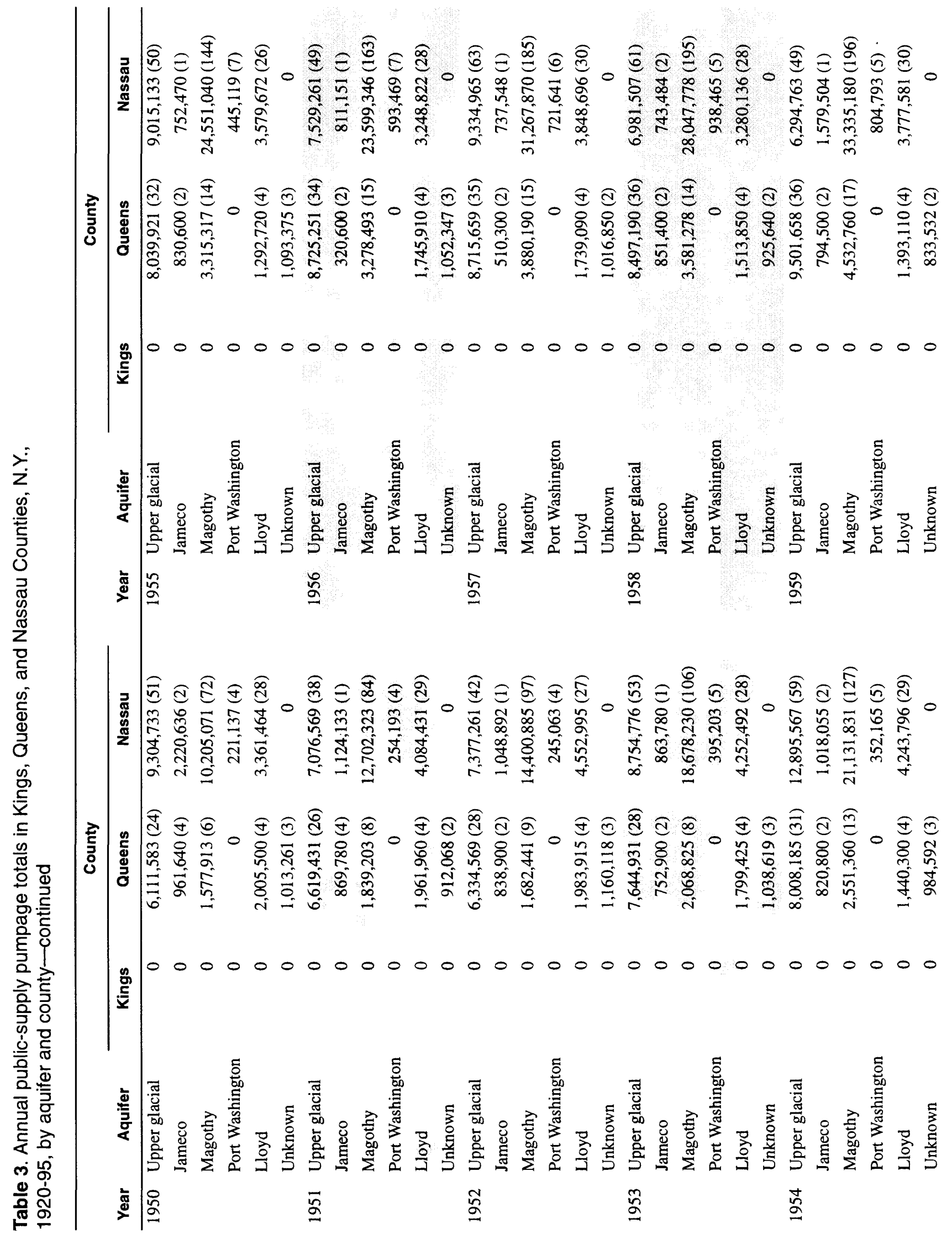




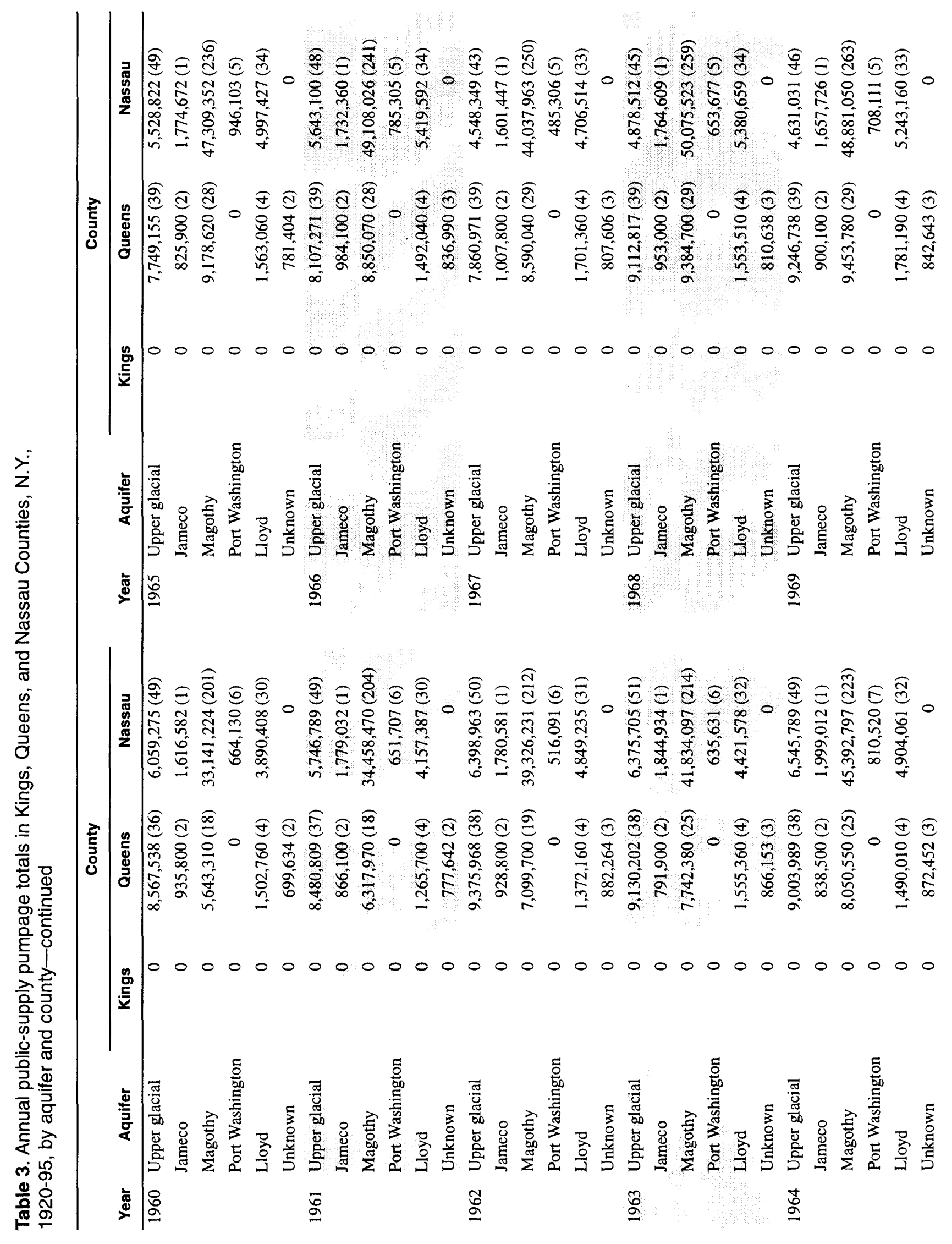




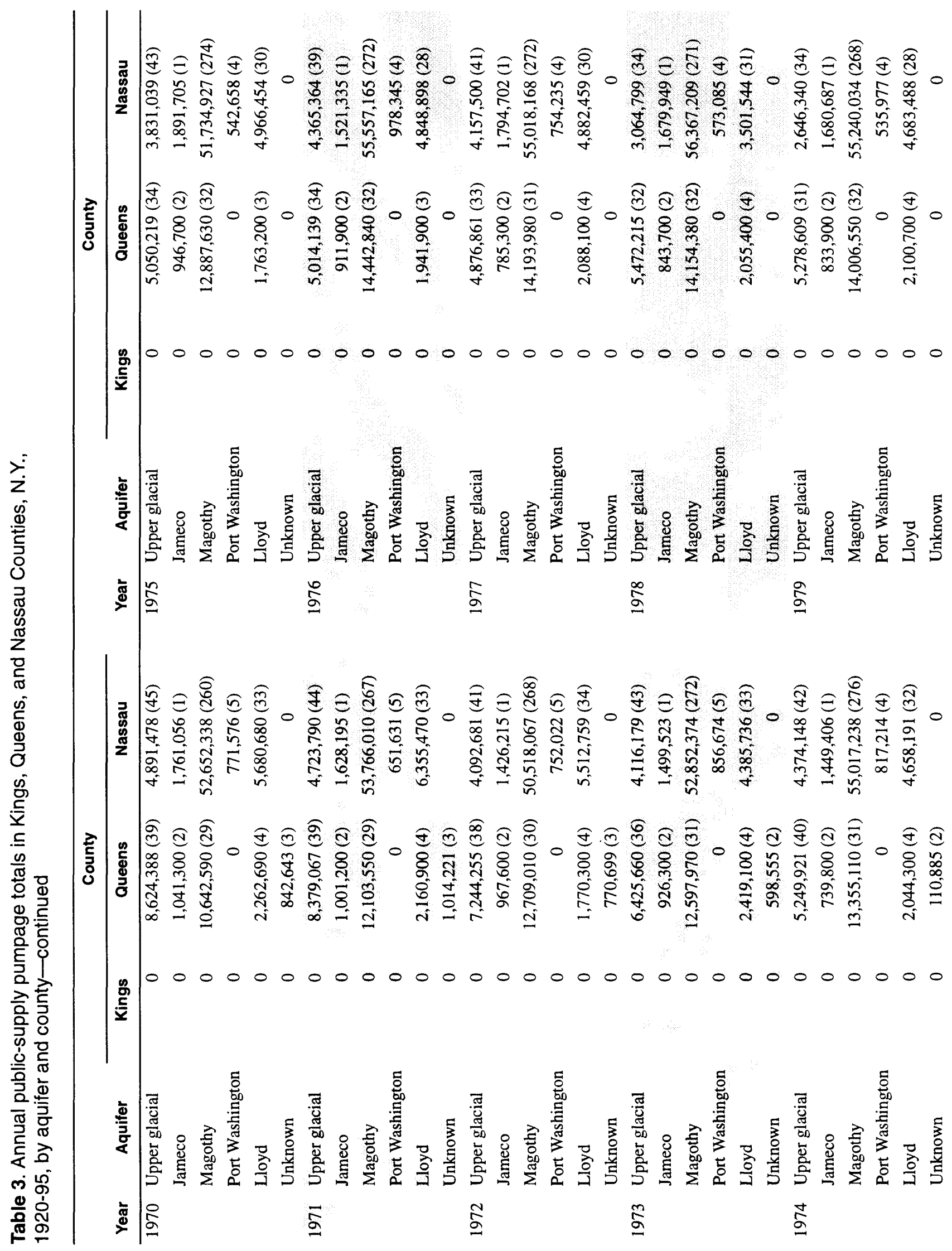




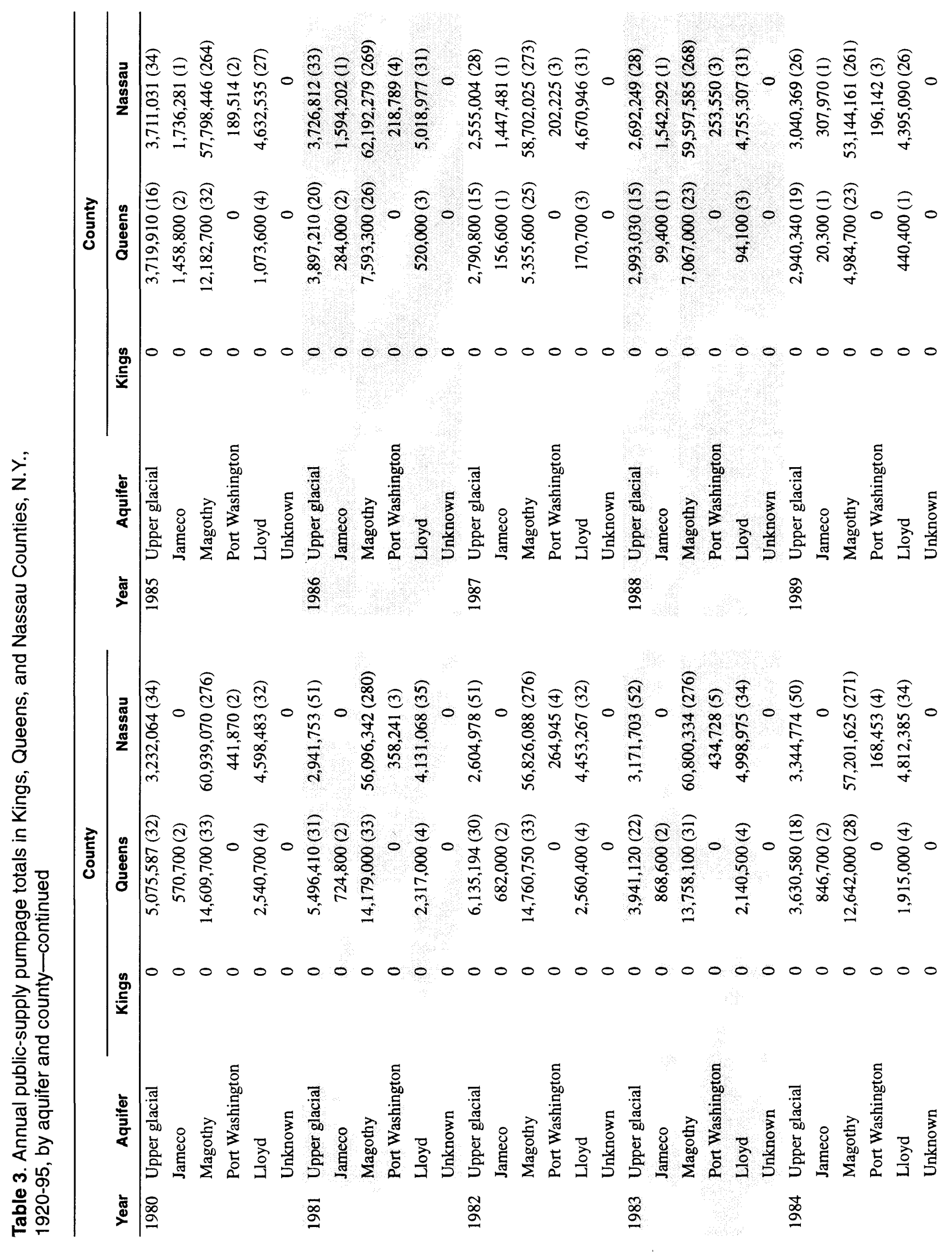




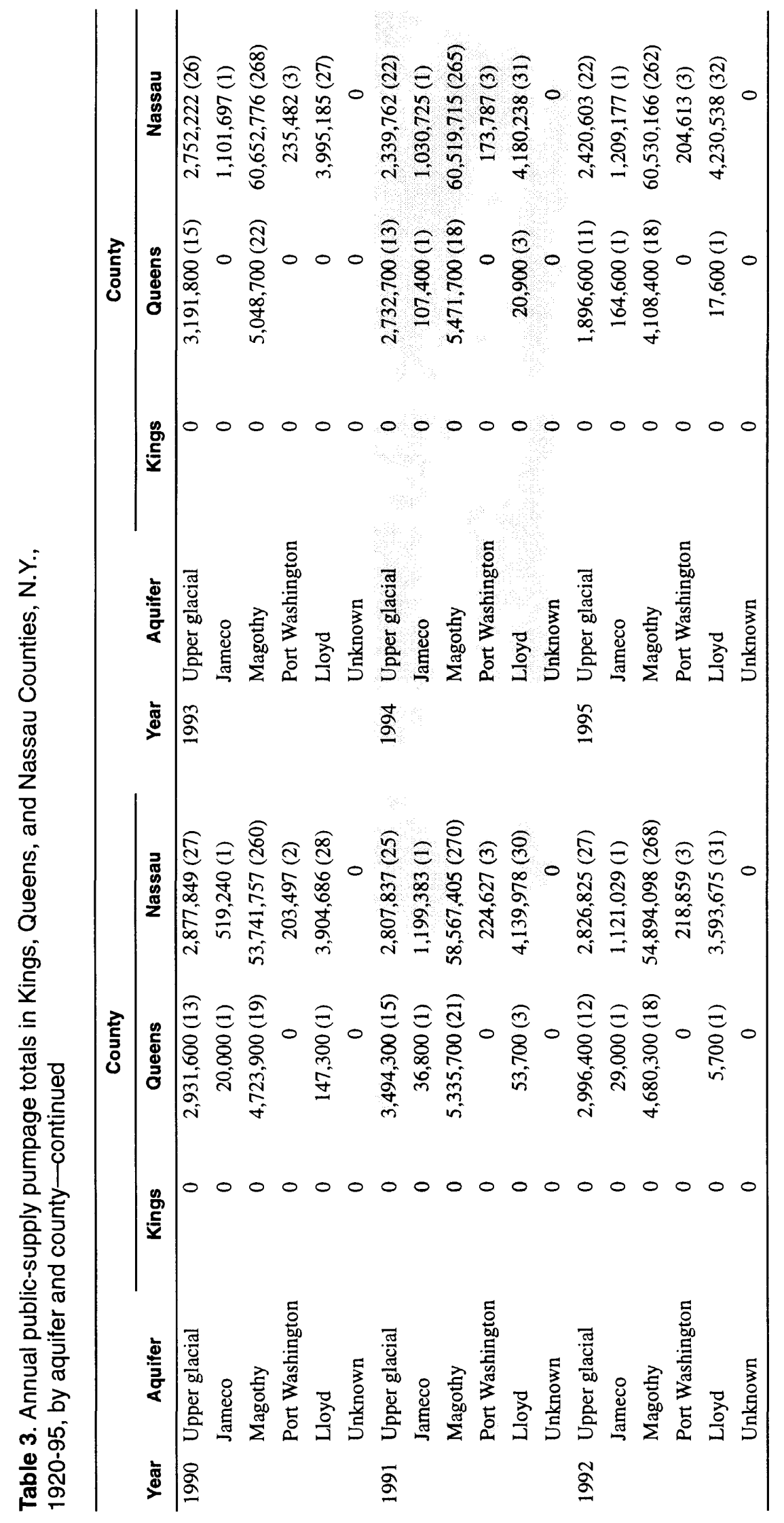


Table 4. Maximum, minimum, and mean annual pumpage in Kings, Queens, and Nassau Counties, N.Y., 1920-95, by county and aquifer [Values are in millions of gallons]

\begin{tabular}{|c|c|c|c|c|c|c|}
\hline \multirow[b]{2}{*}{ County } & \multirow[b]{2}{*}{ Aquifer } & \multicolumn{2}{|c|}{ Maximum } & \multicolumn{2}{|c|}{ Minimum } & \multirow{2}{*}{$\begin{array}{c}\text { Mean } \\
\text { pumpage }{ }^{1}\end{array}$} \\
\hline & & Year & Pumpage & Year & $\overline{\text { Pumpage }^{1}}$ & \\
\hline \multirow[t]{3}{*}{ Kings } & Upper glacial & 1946 & 8,512 & 1932 & 3,232 & 5,650 \\
\hline & Jameco & 1933 & 5,443 & 1927 & 271 & 3,473 \\
\hline & Lloyd & 1931 & 1,105 & 1946 & 11 & 270 \\
\hline \multirow[t]{4}{*}{ Queens } & Upper glacial & 1941 & 11,322 & 1987 & 1,897 & 6,632 \\
\hline & Jameco & 1985 & 1,459 & 1990 & 20 & 719 \\
\hline & Magothy & 1982 & 14,761 & 1926 & 6 & 5,936 \\
\hline & Lloyd & 1944 & 3,021 & 1992 & 6 & 1,515 \\
\hline \multirow[t]{5}{*}{ Nassau } & Upper glacial & 1954 & 12,896 & 1920 & 1,867 & 4,953 \\
\hline & Jameco & 1946 & 2,319 & 1936 & 59 & 1,300 \\
\hline & Magothy & 1986 & 62,192 & 1920 & 199 & 29,648 \\
\hline & Port Washington & 1976 & 978 & 1920 & 14 & 420 \\
\hline & Lloyd & 1971 & 6,355 & 1920 & 250 & 3,311 \\
\hline
\end{tabular}

\footnotetext{
${ }^{1}$ Values in this column do not include years of nonpumping
} 